\title{
Connected sums of constant mean curvature surfaces in Euclidean 3 space.
}

\author{
Rafe Mazzeo* \\ Stanford University
}

\author{
Frank Pacard ${ }^{\dagger}$ \\ Université Paris XII
}

May 11, 1999

\author{
Daniel Pollack $k^{\ddagger}$ \\ University of Washington
}

\section{Introduction and statement of the results}

Amongst the recent developments in the study of embedded complete minimal and constant mean curvature surfaces in $\mathbb{R}^{3}$ is the realization that these objects are far more robust and flexible than is apparent from their Weierstrass representations. Our aim in this paper is to prove a 'gluing theorem', which states roughly that if two (appropriate) constant mean curvature surfaces are juxtaposed, so that their tangent planes are parallel and very close to one another, but oppositely oriented, then there is a new constant mean curvature surface quite near to this configuration (in the Hausdorff topology), but which is a topological connected sum of the two surfaces. We shall explain what we mean by appropriate, or at least give our preliminary interpretation of it, in the next paragraph. Throughout this paper, the acronym CMC shall mean a surface with constant mean curvature equal to one (or minus one depending on the orientation).

The simplest context for our result is when we are given two orientable, immersed, compact CMC surfaces, $\Sigma_{1}$ and $\Sigma_{2}$, with nonempty boundary. Suppose that we have applied a rigid motion to each of these surfaces so that $0 \in \Sigma_{1} \cap \Sigma_{2}$ and $T_{0} \Sigma_{1}=T_{0} \Sigma_{2}$ is the $x y$-plane. (These surfaces may intersect elsewhere, but that is irrelevant for our considerations.) We now define the orientation on these surfaces so that at 0 the oriented unit normal $\nu_{1}$ of $\Sigma_{1}$ equals $(0,0,1)$, while the oriented unit normal $\nu_{2}$ of $\Sigma_{2}$ equals $(0,0,-1)$. Let us assume that with this orientation the two surfaces have the same mean curvature $H_{0}$ (so either $H_{0}=1$ or $H_{0}=-1$ for both of the surfaces). We shall prove that there is a 'geometric connected sum' of these two surfaces, which may be thought of as a desingularization of this configuration. Moreover, the boundary of this desingularization will be the union of the boundaries of the $\Sigma_{i}$, each possibly transformed by a small rigid motion.

In order to state this first result rigorously, we make the following definition:

Definition 1 A compact CMC surface $\Sigma$ with boundary is said to be nondegenerate if there are no Jacobi fields on $\Sigma$ which vanish on $\partial \Sigma$. Namely, if $w: \Sigma \longrightarrow \mathbb{R}$ is a $\mathcal{C}^{2, \alpha}$ solution of

$$
\Delta_{\Sigma} w+\left|\mathbf{A}_{\Sigma}\right|^{2} w=0,\left.\quad w\right|_{\partial \Sigma}=0
$$

then $w=0$. Here $\mathbf{A}_{\Sigma}$ is the second fundamental form of $\Sigma$.

Theorem 1 (Connected sum theorem) Let $\Sigma_{1}$ and $\Sigma_{2}$ be two compact, smooth, immersed, orientable, nondegenerate $C M C$ surfaces with boundary. Assume that these surfaces are positioned and oriented as above and have the same mean curvature $H_{0}$. Then there exist an $\varepsilon_{0}>0$ and a one-parameter family of surfaces $S_{\varepsilon}$, for $\varepsilon \in\left(0, \varepsilon_{0}\right]$, satisfying the following properties:

\footnotetext{
*Supported by the NSF under Grant DMS-9626382

†Supported by AIM (American Institute of Mathematics)

${ }_{\ddagger}$ Supported by the NSF under Grant DMS-9704515
} 
1. $S_{\varepsilon}$ is a smooth, immersed CMC surface with boundary.

2. There are rigid motions $\tau_{1}$ and $\tau_{2}$ of $\mathbb{R}^{3}$, depending on $\varepsilon$, such that $\partial S_{\varepsilon}=\tau_{1}\left(\partial \Sigma_{1}\right) \cup \tau_{2}\left(\partial \Sigma_{2}\right)$.

3. For any fixed $R>0$, the surface $S_{\varepsilon} \cap\left[\mathbb{R}^{3} \backslash B_{R}\right]$ converges in the $\mathcal{C}^{\infty}$ topology to $\left[\Sigma_{1} \cup \Sigma_{2}\right] \cap$ $\left[\mathbb{R}^{3} \backslash B_{R}\right]$ and $\partial S_{\varepsilon}$ converges in the $\mathcal{C}^{\infty}$ topology to $\partial \Sigma_{1} \cup \partial \Sigma_{2}$.

4. The dilated surface $\varepsilon^{-1} S_{\varepsilon}$ converges in the $\mathcal{C}^{\infty}$ topology on any compact set to a catenoid with vertical axis.

Remark 1 There are actually two geometrically distinct families of surfaces $S_{\varepsilon}$ which are constructed here, corresponding to the two choices $H_{0}= \pm 1$. To better visualize these, consider two small spherical caps intersecting at the origin and both tangent to the xy plane. Assume that these surfaces are oriented oppositely to one another so that one is below the xy plane and the other is above. If their normals are pointing outward (so that their mean curvatures are both -1), then the new surfaces $S_{\varepsilon}$ are embedded and very much resemble a neighbourhood of the neck region in an embedded Delaunay surface (an unduloid). Of course, by reversing the orientation of these resulting surfaces we obtain surfaces with mean curvature $=+1$. On the other hand, if the initial orientations are reversed so that the mean curvatures are both +1 , then the resulting $S_{\varepsilon}$ are only immersed, and resemble the neck regions in the immersed Delaunay surfaces of nodoid type.

We also obtain additional geometric information about the surfaces $S_{\varepsilon}$, in particular that their geometry is well-controlled as $\varepsilon \rightarrow 0$.

Proposition 1 (Embeddedness) Under the assumptions of the previous theorem, assume further that $\left[\Sigma_{1} \cup \Sigma_{2}\right] \backslash\{0\}$ is embedded. Then for one of the two choices of $H_{0}$, and for $\varepsilon$ sufficiently small, the surface $S_{\varepsilon}$ is embedded.

We also obtain estimates on the rate of convergence of $S_{\varepsilon}$ to $\Sigma_{1} \cup \Sigma_{2}$.

Proposition 2 (Distance from $\Sigma_{1} \cup \Sigma_{2}$ to $S_{\varepsilon}$ ) Again under the assumptions of the previous theorem, there exists a constant $c>0$ such that

$$
\operatorname{dist}\left(S_{\varepsilon}, \Sigma_{1} \cup \Sigma_{2}\right) \equiv \max \left(\sup _{p \in \Sigma_{1} \cup \Sigma_{2}} \operatorname{dist}\left(p, S_{\varepsilon}\right), \sup _{q \in S_{\varepsilon}} \operatorname{dist}\left(q, \Sigma_{1} \cup \Sigma_{2}\right)\right) \leq c \varepsilon|\log \varepsilon| .
$$

There are various ways to generalize these results. First assume that $\Sigma_{1}$ and $\Sigma_{2}$ are two smooth, oriented CMC surfaces with boundary, which are nondegenerate. If $p_{i} \in \Sigma_{i}, i=1,2$, then we apply a rigid motion to each surface so that $p_{1}=p_{2}=0$ and $T_{0} \Sigma_{i}$ is the $x y$-plane (with opposite orientations as above, and same mean curvature for the two surfaces). Next rotate the surface $\Sigma_{2}$ about the $z$-axis by an angle $\theta \in S^{1}$. This gives a five parameter family of initial configurations $\Sigma_{1} \sqcup \Sigma_{2}\left(p_{1}, p_{2}, \theta\right)$. The precise definition of $\Sigma_{1} \sqcup \Sigma_{2}\left(p_{1}, p_{2}, \theta\right)$ will be given in $\S 16$. Applying Theorem 11 to desingularize each of these configurations adds an additional parameter, and we obtain the six parameter family $S_{\varepsilon}\left(p_{1}, p_{2}, \theta\right)$.

It turns out that this family depends smoothly on all six parameters. We will not prove this explicitly in this paper, in order to keep the technicalities to a minimum; however, the proof is not hard to deduce from our arguments. This dimension count is closely related to the question of whether the solutions $S_{\varepsilon}\left(p_{1}, p_{2}, \theta\right)$ are nondegenerate. For if this is the case for one of these surfaces, then the implicit function theorem gives a six dimensional smooth family of CMC surfaces in a neighbourhood of that surface. Unfortunately we can only prove that these surfaces are nondegenerate for generic choices of parameters. 
Proposition 3 (Generic nondegeneracy property) There is a (singular) codimension one analytic set $\mathcal{S}$ in $\Sigma_{1} \times \Sigma_{2} \times S^{1}$ such that the surface $S_{\varepsilon}\left(p_{1}, p_{2}, \theta\right)$ is nondegenerate provided $\varepsilon$ is sufficiently small and $\left(p_{1}, p_{2}, \varepsilon\right) \notin \mathcal{S}$. The set $\mathcal{S}$ is the union of the locus of points satisfying a quadratic polynomial equation in $\cos \theta$ with coefficients depending on the principal curvatures of the surfaces at $p_{i}$, together with a set $\mathcal{C} \times S^{1}$, where $\mathcal{C}$ is the product of the locus of points on the two surfaces where $\Sigma_{1}$ is umbilic (and hence the principal curvatures are equal to $\left(H_{0} / 2, H_{0} / 2\right)$ ) and the principal curvatures on $\Sigma_{2}$ are equal to $\left(-H_{0} / 2,3 H_{0} / 2\right)$, or vice versa. $\mathcal{C} \times S^{1}$ has dimension less than four unless $\Sigma_{1}$ or $\Sigma_{2}$ is a subdomain of the sphere.

Notice that we have defined the mean curvature to be the sum of the two principal curvatures, not the average.

One important application of this result is that if $S_{\varepsilon}\left(p_{1}, p_{2}, \theta\right)$ is nondegenerate, then one can use it as one of the 'summands' in another application of Theorem 1, and so the connected sum procedure may be iterated. Thus, for example since certain subdomains of the sphere or cylinder with nonempty boundary are nondegenerate, we may glue together arbitrarily many copies of them.

Gluing constructions for geometric objects are by now well understood and even somewhat commonplace, and they have been used to solve a number of diverse problems. Even in the context of CMC and minimal surfaces, there are many results. The pioneering work in this area was that of N. Kapouleas, cf. [4], [5], [6] and [7]. Recently, S.D. Yang [13] has proved a connected sum theorem for complete minimal surfaces of finite total curvature. The methods here could equally well be used to prove that result (or indeed, his methods could be used in the present context), but although Yang requires nondegeneracy of his minimal summands, he does not discuss the question of nondegeneracy of the final surface at all, and it is not clear how it could be obtained by that approach. The issue of nondegeneracy is quite important in the moduli space theory, cf. [8] and the recent work [3].

The results in this paper are also close in spirit to the connected sum theorem in the scalar curvature context in [12], but the methods there are much simpler. The method of proof here is inspired by the recent work of the first and second authors [9] on the construction of CMC surfaces with finitely many Delaunay ends. We now briefly comment on our construction, pointing out its novel features.

The usual steps in such a construction would be to first build a family of approximate solutions, depending on a parameter $\varepsilon>0$. These approximate CMC surfaces would consist of the surfaces $\Sigma_{1}$ and $\Sigma_{2}$ and a catenoidal neck, joined together with cutoff functions, and would converge to the singular configuration $\Sigma_{1} \cup \Sigma_{2}$ as $\varepsilon \rightarrow 0$. They would then be perturbed, when $\varepsilon$ is sufficiently small, to obtain the desingularized CMC surface. This step involves a careful analysis of the Jacobi operator of these approximate solutions, uniformly as $\varepsilon \rightarrow 0$.

We proceed somewhat differently here. Our building blocks are the same, namely the surfaces $\Sigma_{1}$ and $\Sigma_{2}$ and a small 'neck region' of a catenoid. Roughly speaking, we construct perturbations of each of these components which are themselves CMC surfaces with boundary in such a way that the Cauchy data matches across the boundary. The boundary here consists of the small curves produced by excising small balls around the points $p_{1}$ and $p_{2}$ as well as the boundaries of the truncated catenoid. A very important point is that we first perturb each of the surfaces $\Sigma_{i}$ by adding in the normal direction $\varepsilon$ times the Green function for the Jacobi operator with pole at $p_{i}$. This is the precise point where we use nondegeneracy of the surfaces $\Sigma_{i}$, and has the important effect of making the local geometry of $\Sigma_{i}$ near $p_{i}$ insignificant. The catenoid (scaled by $\varepsilon$ ) and these surfaces are then truncated at just the right scale so that their boundaries fit together as well as possible.

In the main step of the construction, we construct the infinite dimensional families of CMC surfaces which are normal graphs over each of these component pieces. This is done by a simple contraction mapping argument. As already intimated, we analyze the Cauchy data of the surfaces in these infinite dimensional families at the boundary curves arising from the truncations. We 
show using degree theory that this Cauchy data may be matched, and hence that the desired CMC surface may be constructed. A substantial advantage of this method is that no extraneous cutoff functions are introduced. Because of the high degree of nonlinearity of the problem, these are typically the cause of many technical complications.

A more detailed guide to the contents is as follows. In $\S \S 3,4$ and 5 we define the truncations of the rescaled catenoid, study the Jacobi operator around these surfaces and construct the family of nearby CMC surfaces, respectively. $\S 6$ collects some facts about the mean curvature operator for graphs and in $\S 7$ we discuss the perturbation of the surfaces $\Sigma_{i}$ by their Green functions. $\S 8$ contains some technical facts about some geometric modifications of these surfaces arising (mostly) from rigid motions. Then in $\S \S 9$ and 10 we study the Jacobi operator on these modified surfaces and then construct the family of nearby CMC surfaces. The Cauchy data maps for each of these components are discussed at the end of $\S \S 5$ and 10 . In the brief $\S 11$ we adapt the previous results to our specific needs. Finally, the degree theory argument for matching the Cauchy data is given in $\S 12$. The remaining sections, $\S \S 13,14$ and 15 , are devoted to the analysis of nondegeneracy. $\S 13$ contains some technical facts which are needed later, certain Jacobi fields on $S_{\varepsilon}$ are discussed in $\S 14$, and using this nondegeneracy is proved in $\S 15$. There are also three brief appendices containing various analytic facts which are required in various places throughout the paper.

The techniques developed here apply immediately to establish a general connected sum theorem for complete, noncompact, embedded CMC surfaces. For such surfaces there is a natural notion of nondegeneracy which in particular follows from the nonexistence of square integrable Jacobi fields (see [8], 11] and [9]). Examples of such surfaces are given by the classical Delaunay surfaces [1] which are CMC surfaces of revolution, and also the surfaces constructed more recently in $[9]$.

In particular, this theorem allows us to glue together any two embedded Delaunay surfaces to produce new embedded four-ended CMC surfaces. As in 12 the resulting surfaces will be asymptotic to the original Delaunay surface on one end of each pair, the other being asymptotic to a small perturbation of the corresponding end (here "small" is understood within the 6 dimensional family of Delaunay surfaces which includes those generated by rigid motions). Since, in this context, nondegeneracy of the resulting surfaces also holds generically, the process may be iterated. This produces families of complete CMC surfaces which are quite different from the previously known examples. Moreover, this nondegeneracy together with the control on the free parameters in our construction allows us to produce an open subset in the moduli space of complete embedded surfaces with $2 k$-ends. This open set is actually a collar neighbourhood of certain boundary components in the moduli space. Precise statements of these results along with applications to the study of the moduli space itself are given in [10].

\section{Notation}

In this brief section we record some notation that will be used frequently, throughout the rest of the paper, and without comment. First, $\lambda: \mathbb{R} \longrightarrow[0,1]$ will denote a smooth cutoff function satisfying

$$
\lambda \equiv 1 \quad \text { if } \quad t>1 \quad \text { and } \quad \lambda \equiv 0 \quad \text { if } \quad t<0 .
$$

Next, if $\phi=\sum_{n \in \mathbb{Z}} a_{n} e^{i n \theta} \in H^{1}\left(S^{1}\right)$, then we define

$$
\left|D_{\theta}\right| \phi \equiv \sum_{n \in \mathbb{Z}}|n| a_{n} e^{i n \theta} \in L^{2}\left(S^{1}\right)
$$

This is, of course, just $\sqrt{-\Delta} \phi$. 
Finally we define orthogonal projections $\pi^{\prime}$ and $\pi^{\prime \prime}$ on $L^{2}\left(S^{1}\right)$ as follows: for

$$
\phi(\theta)=\sum_{n \in \mathbb{Z}} a_{n} e^{i n \theta} \in L^{2}\left(S^{1}\right),
$$

we set

$$
\pi^{\prime}(\phi)=\sum_{|n| \geq 1} a_{n} e^{i n \theta}, \quad \text { and } \quad \pi^{\prime \prime}(\phi)=\sum_{|n| \geq 2} a_{n} e^{i n \theta} \in L^{2}\left(S^{1}\right)
$$

\section{The rescaled catenoids}

The standard catenoid $\Sigma^{c}$ has the following standard parametrization

$$
\mathbf{x}^{c}(s, \theta)=(\cosh s \cos \theta, \cosh s \sin \theta, s), \quad(s, \theta) \in \mathbb{R} \times S^{1} .
$$

$\Sigma^{c}$ may be divided into two pieces, denoted $\Sigma_{ \pm}^{c}$, which are defined to be the image by $\mathbf{x}^{c}$ of $\left(\mathbb{R}^{ \pm} \times S^{1}\right)$, respectively. We may also parameterize the lower half $\Sigma_{-}^{c}$ by

$$
\mathbb{R}^{2} \backslash B_{1} \ni(x, y) \longrightarrow\left(x, y,-\log r-\log 2+\mathcal{O}\left(r^{-2}\right)\right) \quad \text { as } r \rightarrow \infty .
$$

Here, as usual, $r=\left(x^{2}+y^{2}\right)^{1 / 2}$. For any $\varepsilon>0$, we define the rescaled catenoid $\Sigma_{\varepsilon}^{c}$ by scaling $\Sigma^{c}$ by the factor $\varepsilon$ and translating by $-\varepsilon \log \varepsilon+\varepsilon \log 2$ along the $z$-axis. $\Sigma_{\varepsilon}^{c}$ is parameterized by

$$
\mathbf{x}_{\varepsilon}^{c}(s, \theta)=(\varepsilon \cosh s \cos \theta, \varepsilon \cosh s \sin \theta, \varepsilon s-\varepsilon \log \varepsilon+\varepsilon \log 2),(s, \theta) \in \mathbb{R} \times S^{1} .
$$

Again $\Sigma_{\varepsilon}^{c}$ decomposes into two pieces, $\Sigma_{\varepsilon, \pm}^{c}$. By (5) we may parametrize $\Sigma_{\varepsilon,-}^{c}$ either as

$$
\mathbb{R}^{2} \backslash B_{1} \ni(x, y) \longrightarrow\left(\varepsilon x, \varepsilon y,-\varepsilon \log r-\varepsilon \log \varepsilon+\mathcal{O}\left(\varepsilon r^{-2}\right)\right),
$$

or equivalently (replacing $(\varepsilon x, \varepsilon y)$ by $(x, y))$,

$$
\mathbb{R}^{2} \backslash B_{\varepsilon} \ni(x, y) \longrightarrow\left(x, y,-\varepsilon \log r+\mathcal{O}\left(\varepsilon^{3} r^{-2}\right)\right) .
$$

The simplicity of this final parametrization is why we introduced the translation along the $z$-axis in the first place.

Finally, consider all surfaces near to the rescaled catenoid $\Sigma_{\varepsilon}^{c}$ which may be written as normal graphs off of it. Since the outer unit normal of $\Sigma_{\varepsilon}^{c}$ is given by

$$
n(s, \theta)=\frac{1}{\cosh s}(\cos \theta, \sin \theta,-\sinh s),
$$

each of these surfaces may parameterized as

$$
(s, \theta) \longrightarrow \mathbf{x}_{\varepsilon}^{c}(s, \theta)+w(s, \theta) n(s, \theta),
$$

for some function $w \in \mathcal{C}^{2}\left(\mathbb{R}^{-} \times S^{1}\right)$, which is suitably small. We prove in Appendix II that the linearized mean curvature operator about $\Sigma_{\varepsilon}^{c}$, i.e. at $w=0$, is given by $-(\varepsilon \cosh s)^{-2} \mathcal{L}$, where

$$
\mathcal{L} w \equiv \partial_{s s}^{2} w+\partial_{\theta \theta}^{2} w+\frac{2}{\cosh ^{2} s} w .
$$

As usual, we call this the Jacobi operator. We also prove in this appendix that the mean curvature of the surface parameterized by (9) is given by an expression of the form

$$
\begin{aligned}
H_{w}=-\frac{1}{\varepsilon^{2} \cosh ^{2} s} \mathcal{L} w & +\frac{1}{\varepsilon \cosh ^{2} s} Q_{\varepsilon}^{\prime}\left(\frac{w}{\varepsilon \cosh s}, \frac{\nabla w}{\varepsilon \cosh s}, \frac{\nabla^{2} w}{\varepsilon \cosh s}\right) \\
& +\frac{1}{\varepsilon \cosh s} Q_{\varepsilon}^{\prime \prime}\left(\frac{w}{\varepsilon \cosh s}, \frac{\nabla w}{\varepsilon \cosh s}, \frac{\nabla^{2} w}{\varepsilon \cosh s}\right),
\end{aligned}
$$


where $Q_{\varepsilon}^{\prime}$ and $Q_{\varepsilon}^{\prime \prime}$ are functions which are bounded in $\mathcal{C}^{k}\left(\left[-s_{\varepsilon}, s_{\varepsilon}\right] \times S^{1}\right)$ for all $k$, uniformly in $\varepsilon$. These functions also satisfy

$$
Q_{\varepsilon}^{\prime}(0,0,0)=Q_{\varepsilon}^{\prime \prime}(0,0,0)=0 \quad \text { and } \quad \nabla Q_{\varepsilon}^{\prime}(0,0,0)=\nabla Q_{\varepsilon}^{\prime \prime}(0,0,0)=0,
$$

and in addition

$$
\nabla^{2} Q_{\varepsilon}^{\prime \prime}(0,0,0)=0
$$

\section{The mean curvature operator linearized about the trun- cated catenoid}

In the next section we shall study the space of CMC surfaces in a neighbourhood of the truncated catenoid. This analysis depends on a good understanding of the linearization of the mean curvature operator, or equivalently, of the operator $\mathcal{L}$ of (10), on arbitrarily large truncations of the catenoid. We consider this now.

The mapping properties of $\mathcal{L}$ are best stated in terms of the following weighted spaces:

Definition 2 For each $k \in \mathbb{N}$ and $\alpha \in(0,1)$, let $|w|_{k, \alpha,[s, s+1]}$, denote the usual $\mathcal{C}^{k, \alpha}$ Hölder norm on the set $[s, s+1] \times S^{1}$. Then for any $\delta \in \mathbb{R}$,

$$
\mathcal{C}_{\delta}^{k, \alpha}\left(\mathbb{R} \times S^{1}\right)=\left\{w \in \mathcal{C}_{\text {loc }}^{k, \alpha}\left(\mathbb{R} \times S^{1}\right):\|w\|_{k, \alpha, \delta} \equiv \sup _{s \in \mathbb{R}}\left[(\cosh s)^{-\delta}|w|_{k, \alpha,[s, s+1]}\right]<\infty\right\} .
$$

For any closed interval $I \subset \mathbb{R}$, we denote the restriction of $\mathcal{C}_{\delta}^{k, \alpha}\left(\mathbb{R} \times S^{1}\right)$ to $I \times S^{1}$ by $\mathcal{C}_{\delta}^{k, \alpha}\left(I \times S^{1}\right)$, endowed with the induced norm.

Proposition 4 Fix $\delta \in(1,2)$. Then for any $s_{0} \in \mathbb{R}^{+}$there exists an operator

$$
\mathcal{G}_{s_{0}}: \mathcal{C}_{\delta}^{0, \alpha}\left(\left[-s_{0}, s_{0}\right] \times S^{1}\right) \longrightarrow \mathcal{C}_{\delta}^{2, \alpha}\left(\left[-s_{0}, s_{0}\right] \times S^{1}\right)
$$

such that for any $f \in \mathcal{C}_{\delta}^{0, \alpha}\left(\left[-s_{0}, s_{0}\right] \times S^{1}\right)$, the function $w=\mathcal{G}_{s_{0}}(f)$ solves

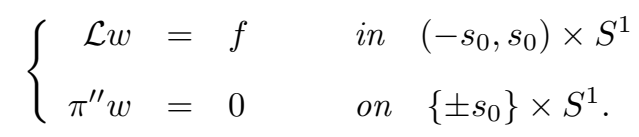

Moreover, $\left\|\mathcal{G}_{s_{0}}(f)\right\|_{2, \alpha, \delta} \leq c\|f\|_{0, \alpha, \delta}$, for some constant $c>0$ independent of $s_{0}$.

Remark 2 The right inverse $\mathcal{G}_{s_{0}}$ with these properties is not uniquely defined. We shall always use the one constructed in the proof below.

Proof: Assume that $|f(s, \theta)| \leq(\cosh s)^{\delta}$. Now decompose both $w$ and $f$ into Fourier series

$$
w=\sum_{n \in \mathbb{Z}} w_{n}(s) e^{i n \theta} \quad \text { and } \quad f=\sum_{n \in \mathbb{Z}} f_{n}(s) e^{i n \theta} .
$$

For $|n| \geq 2, w_{n}$ must solve

$$
\ddot{w}_{n}-n^{2} w_{n}+\frac{2}{\cosh ^{2} s} w_{n}=f_{n} \quad \text { in } \quad|s|<s_{0}, \quad w_{n}\left( \pm s_{0}\right)=0 .
$$

The dots represent differentiation with respect to $s$. 
Since $|n| \geq 2$,

$$
L_{n}=\frac{d^{2}}{d s^{2}}-n^{2}+\frac{2}{\cosh ^{2} s}
$$

satisfies the maximum principle, so that if $w$ is defined on some interval $\left[s_{1}, s_{2}\right] \subset \mathbb{R}$ and if $w\left(s_{1}\right) \geq 0, w\left(s_{2}\right) \geq 0$ and $L_{n} w \leq 0$ on $\left(s_{1}, s_{2}\right)$, then $w \geq 0$ in $\left[s_{1}, s_{2}\right]$. We obtain the solution of $L_{n} w_{n}=f_{n}$ by the method of sub- and supersolutions once we have constructed an appropriate barrier function. But

$$
L_{n}(\cosh s)^{\delta}=\left(\left(\delta^{2}-n^{2}\right) \cosh ^{2} s+2+\delta-\delta^{2}\right)(\cosh s)^{\delta-2},
$$

and then, since $\delta \in(1,2)$,

$$
\left(\delta^{2}-n^{2}\right) \cosh ^{2} s+2+\delta-\delta^{2} \leq-\left(n^{2}-2-\delta\right) \cosh ^{2} s .
$$

Therefore, since $\left|f_{n}(s)\right| \leq(\cosh s)^{\delta}$, we have that

$$
\begin{aligned}
& L_{n}\left(w_{n}-\left(n^{2}-2-\delta\right)^{-1} \cosh ^{\delta} s\right) \geq 0 \\
& L_{n}\left(w_{n}+\left(n^{2}-2-\delta\right)^{-1} \cosh ^{\delta} s\right) \leq 0 .
\end{aligned}
$$

We conclude that the solution $w_{n}$ exists and satisfies

$$
\left|w_{n}(s)\right| \leq \frac{1}{n^{2}-2-\delta}(\cosh s)^{\delta} .
$$

Next we obtain the solution and estimates when $n=0, \pm 1$. This is straightforward since we know homogeneous solutions of $L_{n}$ explicitly for these values of $n$. In fact, $L_{0} \tanh s=0$ and $L_{ \pm 1}(\cosh s)^{-1}=0$. Therefore, by 'variation of constants', we obtain the solutions

$$
w_{0}(s)=\tanh s \int_{0}^{s} \tanh ^{-2} t \int_{0}^{t} \tanh u f_{0}(u) d u d t,
$$

and

$$
w_{ \pm 1}(s)=\cosh ^{-1} s \int_{0}^{s} \cosh ^{2} t \int_{0}^{t} \cosh ^{-1} u f_{ \pm 1}(u) d u d t .
$$

Straightforward estimates using these formulæ and the fact that $\left|f_{n}(s)\right| \leq(\cosh s)^{\delta}, n=0, \pm 1$, gives

$$
\left|w_{0}(s)\right|+\left|w_{ \pm 1}(s)\right| \leq c(\cosh s)^{\delta},
$$

for some constant $c>0$ independent of $s_{0}$.

To finish the proof we must amalgamate these estimates. But the coefficient on the right in (15) is summable in $n$, and so we easily see that $|w(s, \theta)| \leq c(\cosh s)^{\delta}$. The estimates for the derivatives of $w$ are then obtained by Schauder theory.

Using a similar technique, we prove the

Proposition 5 For each $s_{0}>0$ there exists an operator

$$
\mathcal{P}_{s_{0}}^{0}:\left(\pi^{\prime \prime}\left(\mathcal{C}^{2, \alpha}\left(S^{1}\right)\right)\right)^{2} \longrightarrow \mathcal{C}_{2}^{2, \alpha}\left(\left[-s_{0}, s_{0}\right] \times S^{1}\right)
$$

such that for all $\phi_{ \pm}^{\prime \prime} \in \pi^{\prime \prime}\left(\mathcal{C}^{2, \alpha}\left(S^{1}\right)\right)$, the function $w=\mathcal{P}_{s_{0}}^{0}\left(\phi_{+}^{\prime \prime}, \phi_{-}^{\prime \prime}\right)$ solves

$$
\left\{\begin{aligned}
\Delta w & =0 & \text { in } & \left(-s_{0}, s_{0}\right) \times S^{1} \\
w & =\phi_{ \pm}^{\prime \prime} & \text { on } & \left\{ \pm s_{0}\right\} \times S^{1} .
\end{aligned}\right.
$$

We also have $\left\|\mathcal{P}_{s_{0}}^{0}\left(\phi_{+}, \phi_{-}\right)\right\|_{2, \alpha, 2} \leq c e^{-2 s_{0}}\left(\left\|\phi_{+}^{\prime \prime}\left|\left\|_{2, \alpha}+\right\| \phi_{-}^{\prime \prime}\right|_{2, \alpha}\right)\right.$ for some $c>0$ independent of $s_{0}$. 
Proof: By linearity, we may assume that $\left\|\phi_{+}^{\prime \prime}\right\|_{2, \alpha}+\left\|\phi_{-}^{\prime \prime}\right\|_{2, \alpha} \leq 1$. Again, we decompose $w$ into Fourier series

$$
w=\sum_{|n| \geq 2} w_{n}(s) e^{i n \theta}
$$

and obtain the solution by the method of sub- and supersolutions once we have constructed an appropriate barrier function. But

$$
\Delta\left((\cosh s)^{n} e^{i n \theta}\right)=-n(n-1)(\cosh s)^{n-2} \leq 0 .
$$

Therefore, $s \rightarrow\left(\cosh s_{0}\right)^{-n}(\cosh s)^{n}$ can be used as a barrier function. We conclude that the solution $w_{n}$ exists and satisfies

$$
\left|w_{n}(s)\right| \leq\left(\cosh s_{0}\right)^{-n}(\cosh s)^{n} .
$$

From this it is easy to get the estimate

$$
|w(s, \theta)| \leq c\left(\cosh s_{0}\right)^{-2}(\cosh s)^{2}
$$

for all $|s| \leq s_{0}-1$. The rest of the proof is now obvious and left to the reader.

Using the previous results, we also get

Proposition 6 Fix $\delta \in(1,2)$. Then for each $s_{0}>0$ there exists an operator

$$
\mathcal{P}_{s_{0}}:\left(\pi^{\prime \prime}\left(\mathcal{C}^{2, \alpha}\left(S^{1}\right)\right)\right)^{2} \longrightarrow \mathcal{C}_{\delta}^{2, \alpha}\left(\left[-s_{0}, s_{0}\right] \times S^{1}\right)
$$

such that for all $\phi_{ \pm}^{\prime \prime} \in \pi^{\prime \prime}\left(\mathcal{C}^{2, \alpha}\left(S^{1}\right)\right)$, the function $w=\mathcal{P}_{s_{0}}\left(\phi_{+}^{\prime \prime}, \phi_{-}^{\prime \prime}\right)$ solves

$$
\left\{\begin{aligned}
\mathcal{L} w=0 & \text { in } \quad\left(-s_{0}, s_{0}\right) \times S^{1} \\
w=\phi_{ \pm}^{\prime \prime} & \text { on }\left\{ \pm s_{0}\right\} \times S^{1} .
\end{aligned}\right.
$$

We also have $\left\|\left(\mathcal{P}_{s_{0}}-\mathcal{P}_{s_{0}}^{0}\right)\left(\phi_{+}, \phi_{-}\right)\right\|_{2, \alpha, \delta} \leq c e^{-2 s_{0}}\left(\left\|\phi_{+}^{\prime \prime}||_{2, \alpha}+\right\| \phi_{-}^{\prime \prime} \|_{2, \alpha}\right)$ for some $c>0$ independent of $s_{0}$.

Proof: Set $w=w_{0}+v$ where the functions $w_{0}$ and $v$ are given by $w_{0}=\mathcal{P}_{s_{0}}^{0}\left(\phi_{+}^{\prime \prime}, \phi_{-}^{\prime \prime}\right)$ and $v=-\mathcal{G}_{s_{0}} \mathcal{L} w_{0}=-2 \mathcal{G}_{s_{0}}\left((\cosh s)^{-2} w_{0}\right), v\left( \pm s_{0}, \theta\right)=0$. Then the estimate $\left\|w_{0}\right\|_{2, \alpha, 2} \leq$ $c e^{-2 s_{0}}\left(\left\|\phi_{+}^{\prime \prime}\right\|_{2, \alpha}+\left\|\phi_{-}^{\prime \prime}\right\|_{2, \alpha}\right)$ and an application of Proposition 1 give the estimate for $v$ and finishes the proof.

To simply notation we shall henceforth write $\mathcal{P}_{s_{0}}^{0}\left(\phi_{ \pm}^{\prime \prime}\right), \mathcal{P}_{s_{0}}\left(\phi_{ \pm}^{\prime \prime}\right)$ and $\left\|\phi_{ \pm}^{\prime \prime}\right\|_{2, \alpha}$ in place of the longer versions above.

\section{CMC surfaces near the truncated catenoid}

Now and hereafter, we set

$$
s_{\varepsilon}=-\frac{1}{4} \log \varepsilon .
$$

Use the parametrization (6) for the rescaled catenoid. Its outer unit normal $n(s, \theta)$ at $\mathbf{x}_{\varepsilon}^{c}(s, \theta)$ is then given by (8). Define a smooth, strictly monotone function $\xi_{\varepsilon}: \mathbb{R} \longrightarrow[-1,1]$ by

$$
\xi_{\varepsilon}(s)=-\left(1-\lambda\left(s_{\varepsilon}-1-|s|\right)\right) \frac{s}{|s|}-\lambda\left(s_{\varepsilon}-1-|s|\right) \tanh s,
$$

Thus $\xi_{\varepsilon}(s)=-\frac{s}{|s|}$ for $|s| \geq s_{\varepsilon}-1$ and $\xi_{\varepsilon}(s)=-\tanh s$ for $|s| \leq s_{\varepsilon}-2$. Now consider the vector field

$$
\bar{n}_{\varepsilon}(s, \theta)=\left(\sqrt{1-\xi_{\varepsilon}^{2}(s)} \cos \theta, \sqrt{1-\xi_{\varepsilon}^{2}(s)} \sin \theta, \xi_{\varepsilon}(s)\right)
$$


this is a perturbation of the unit normal $n$, and in fact

$$
\bar{n}_{\varepsilon}(s, \theta)-n(s, \theta)=\left(\chi_{\varepsilon}(s) \cos \theta, \chi_{\varepsilon}(s) \sin \theta, \bar{\chi}_{\varepsilon}(s)\right) .
$$

where $\chi_{\varepsilon}$ and $\bar{\chi}_{\varepsilon}$ are supported in $\left(-\infty,-s_{\varepsilon}+2\right] \cup\left[s_{\varepsilon}-2,+\infty\right)$ and satisfy

$$
\cosh s\left|\nabla^{k} \chi_{\varepsilon}\right|+\cosh ^{2} s\left|\nabla^{k} \bar{\chi}_{\varepsilon}\right| \leq c_{k},
$$

for all $k \geq 0$.

We now look for all CMC surfaces near the rescaled catenoid which admit the parametrization

$$
\mathbf{x}_{w}:\left[-s_{\varepsilon}, s_{\varepsilon}\right] \times S^{1} \ni(s, \theta) \longrightarrow \mathbf{x}_{\varepsilon}^{c}(s, \theta)+w(s, \theta) \bar{n}_{\varepsilon}(s, \theta),
$$

for some smooth, sufficiently small function $w$. By construction, these surfaces are normal graphs over $\Sigma_{\varepsilon}^{c}$ when $|s| \leq s_{\varepsilon}-2$ and are vertical graphs when $|s| \geq s_{\varepsilon}-1$. The reason for parametrizing surfaces using $\bar{n}_{\varepsilon}$ is so that their boundaries are vertical graphs over a circle. It follows from the analysis of Appendix II and from (11) that such a surface is CMC if and only if $w$ satisfies a certain nonlinear equation of the form

$$
\frac{1}{\varepsilon^{2} \cosh ^{2} s} \mathcal{L} w=\frac{1}{\varepsilon^{2} \cosh ^{2} s}\left(-H_{0} \varepsilon^{2} \cosh ^{2} s+\bar{Q}_{\varepsilon}(w)\right),
$$

where

$$
\begin{aligned}
\bar{Q}_{\varepsilon}(w) & =L_{\varepsilon} w+\varepsilon \bar{Q}_{\varepsilon}^{\prime}\left(\frac{w}{\varepsilon \cosh s}, \frac{\nabla w}{\varepsilon \cosh s}, \frac{\nabla^{2} w}{\varepsilon \cosh s}\right) \\
& +\varepsilon \cosh s \bar{Q}_{\varepsilon}^{\prime \prime}\left(\frac{w}{\varepsilon \cosh s}, \frac{\nabla w}{\varepsilon \cosh s}, \frac{\nabla^{2} w}{\varepsilon \cosh s}\right) .
\end{aligned}
$$

Here $\bar{Q}_{\varepsilon}^{\prime}$ and $\bar{Q}_{\varepsilon}^{\prime \prime}$ have the same properties (12) and (13) as the functions $Q_{\varepsilon}^{\prime}$ and $Q_{\varepsilon}^{\prime \prime}$ in (11) and the linear operator $L_{\varepsilon}$ is supported in $|s| \geq s_{\varepsilon}-2$, has coefficients of the order $1 / \cosh ^{2} s$, and represents the difference between the Jacobi operator for surfaces parametrized normally to $\Sigma_{\varepsilon}^{c}$ and those parametrized using $\bar{n}_{\varepsilon}$. (To see this estimate on the size of the coefficients of $L_{\varepsilon}$, note from (88) in Appendix I that this difference involves $1-n \cdot \bar{n}_{\varepsilon}$, which by (25) and (26) is of order $1 / \cosh ^{2} s$.)

Now, given $\phi_{ \pm}^{\prime \prime} \in \pi^{\prime \prime}\left(\mathcal{C}^{2, \alpha}\left(S^{1}\right)\right)$, we wish to solve the boundary value problem

$$
\left\{\begin{aligned}
\mathcal{L} w & =-H_{0} \varepsilon^{2} \cosh ^{2} s+\bar{Q}_{\varepsilon}(w) & & \text { in } & & \left(-s_{\varepsilon}, s_{\varepsilon}\right) \times S^{1} \\
\pi^{\prime \prime} w & =\phi_{ \pm}^{\prime \prime} & & \text { on } & & \left\{ \pm s_{\varepsilon}\right\} \times S^{1} .
\end{aligned}\right.
$$

A solution will produce a CMC surface with boundary components parametrized by

$$
S^{1} \ni \theta \longrightarrow\left(\varepsilon \cosh s_{\varepsilon} \cos \theta, \varepsilon \cosh s_{\varepsilon} \sin \theta, \pm \varepsilon s_{\varepsilon}-\varepsilon \log \varepsilon+\varepsilon \log 2 \mp w\left( \pm s_{\varepsilon}, \theta\right)\right) .
$$

Note these are vertical graphs over (small) circles.

We solve (29) by a standard contraction mapping argument. First fix $\delta \in(1,2)$ and define

$$
\tilde{w}=\mathcal{P}_{s_{\varepsilon}}\left(\phi_{ \pm}^{\prime \prime}\right)-H_{0} \mathcal{G}_{s_{\varepsilon}}\left(\varepsilon^{2} \cosh ^{2} s\right)
$$

as an approximate solution for the problem. Then, writing $w=\tilde{w}+v$, we must find a function $v \in \mathcal{C}_{\delta}^{2, \alpha}\left(\left[-s_{\varepsilon}, s_{\varepsilon}\right] \times S^{1}\right)$ such that

$$
\left\{\begin{aligned}
\mathcal{L} v & =\bar{Q}_{\varepsilon}(\tilde{w}+v) & & \text { in } & & \left(-s_{\varepsilon}, s_{\varepsilon}\right) \times S^{1} \\
\pi^{\prime \prime} v & =0 & & \text { on } & & \left\{ \pm s_{\varepsilon}\right\} \times S^{1} .
\end{aligned}\right.
$$

This will be accomplished by finding a fixed point of the mapping

$$
\mathcal{N}_{\varepsilon}(v) \equiv \mathcal{G}_{s_{\varepsilon}}\left(\bar{Q}_{\varepsilon}(\tilde{w}+v)\right)
$$

Although not explicit in the notation, this operator depends on $\phi_{ \pm}^{\prime \prime}$. 
Proposition 7 Fix $\kappa>0$. Then there exist constants $c_{\kappa}>0$ and $\varepsilon_{0}>0$ such that if $0<\varepsilon \leq \varepsilon_{0}$ and if $\phi_{ \pm}^{\prime \prime} \in \pi^{\prime \prime}\left(\mathcal{C}^{2, \alpha}\left(S^{1}\right)\right)$ is fixed with $\left\|\phi_{ \pm}^{\prime \prime}\right\|_{2, \alpha} \leq \kappa \varepsilon^{3 / 2}$, then $\mathcal{N}_{\varepsilon}$ is a contraction mapping on the ball

$$
B_{c_{\kappa}} \equiv\left\{v:\|v\|_{2, \alpha, \delta} \leq 2 c_{\kappa} \varepsilon^{(8+\delta) / 4}\right\},
$$

and hence has a unique fixed point in this ball.

Proof: We must show that

$$
\left\|\mathcal{N}_{\varepsilon}(0)\right\|_{2, \alpha, \delta} \leq c_{\kappa} \varepsilon^{(8+\delta) / 4}
$$

and

$$
\left\|\mathcal{N}_{\varepsilon}\left(v_{2}\right)-\mathcal{N}_{\varepsilon}\left(v_{1}\right)\right\|_{2, \alpha, \delta} \leq \frac{1}{2}\left\|v_{2}-v_{1}\right\|_{2, \alpha, \delta},
$$

for all $v_{1}, v_{2} \in B_{c_{\kappa}}$. For then, if $v \in B_{c_{\kappa}}$, then

$$
\|N(v)\|_{2, \alpha, \delta} \leq\|N(0)\|_{2, \alpha, \delta}+\|N(v)-N(0)\|_{2, \alpha, \delta} \leq 2 c_{\kappa} \varepsilon^{(8+\delta) / 4} .
$$

We begin with the first of these. To do this, we must estimate $\tilde{w}$. Set $\tilde{w}_{0}=\mathcal{P}_{s_{\varepsilon}}^{0}\left(\phi_{ \pm}^{\prime \prime}\right)$; then since $e^{-2 s_{\varepsilon}}=\varepsilon^{1 / 2}$ and $\left\|\phi_{ \pm}^{\prime \prime}\right\|_{2, \alpha} \leq \kappa \varepsilon^{3 / 2}$, we get from Proposition 5 and Proposition 6 that

$$
\left\|\tilde{w}_{0}\right\|_{2, \alpha, 2}+\left\|\mathcal{P}_{s_{\varepsilon}}\left(\phi_{ \pm}^{\prime \prime}\right)-\tilde{w}_{0}\right\|_{2, \alpha, \delta} \leq c \kappa \varepsilon^{2} .
$$

Next, even if the result of Proposition 1 does not hold when the weight parameter $\delta=2$ and taking advantage of the fact that $\varepsilon^{2} \cosh ^{2} s$ is independent of $\theta$, we can use directly (16), to estimate

$$
\left\|\mathcal{G}_{s_{\varepsilon}}\left(\varepsilon^{2} \cosh ^{2} s\right)\right\|_{2, \alpha, 2} \leq c \varepsilon^{2} .
$$

Notice that $\|\cdot\|_{2, \alpha, 2} \leq\|\cdot\|_{2, \alpha, \delta}$, since $\delta \in(1,2)$. Putting these together, we get

$$
\|\tilde{w}\|_{2, \alpha, 2} \leq c \varepsilon^{2}
$$

for some constant $c$ depending on $\kappa$ but independent of $\varepsilon$.

Next we estimate $\left\|\mathcal{N}_{\varepsilon}(0)\right\|_{2, \alpha, \delta}$ by

$$
\left\|\mathcal{N}_{\varepsilon}(0)\right\|_{2, \alpha, \delta} \leq c\left(\left\|L_{\varepsilon} \tilde{w}\right\|_{0, \alpha, \delta}+\left\|\varepsilon \bar{Q}_{\varepsilon}^{\prime}(\tilde{w} / \varepsilon \cosh s)\right\|_{0, \alpha, \delta}+\left\|\varepsilon \cosh s \bar{Q}_{\varepsilon}^{\prime \prime}(\tilde{w} / \varepsilon \cosh s)\right\|_{0, \alpha, \delta}\right) .
$$

We have first

$$
\left\|L_{\varepsilon} \tilde{w}\right\|_{0, \alpha, \delta} \leq c \varepsilon^{(8+\delta) / 4}
$$

and then

$$
\left\|\varepsilon \bar{Q}_{\varepsilon}^{\prime}\left(\frac{\tilde{w}}{\varepsilon \cosh s}\right)\right\|_{0, \alpha, \delta} \leq c \varepsilon^{(10+\delta) / 4} \quad \text { and } \quad\left\|\varepsilon \cosh s \bar{Q}_{\varepsilon}^{\prime \prime}\left(\frac{\tilde{w}}{\varepsilon \cosh s}\right)\right\|_{0, \alpha, \delta} \leq c \varepsilon^{(12+\delta) / 4}
$$

Again, all constants depend on $\kappa$ but not on $\varepsilon$.

Now clearly it suffices to choose $\varepsilon$ sufficiently small and $c_{\kappa}$ equal to twice the constant in (35) in order for the stated estimate for $\mathcal{N}_{\varepsilon}(0)$ to hold.

For the other estimate, if $v_{1}, v_{2} \in B_{c_{\kappa}}$, then

$$
\begin{gathered}
\left\|\mathcal{G}_{s_{\varepsilon}} L_{\varepsilon}\left(v_{1}-v_{2}\right)\right\|_{2, \alpha, \delta} \leq c\left\|L_{\varepsilon}\left(v_{2}-v_{1}\right)\right\|_{0, \alpha, \delta} \leq c \varepsilon^{1 / 2}\left\|v_{2}-v_{1}\right\|_{2, \alpha, \delta}, \\
\left\|\varepsilon\left(\bar{Q}_{\varepsilon}^{\prime}\left(\frac{\tilde{w}+v_{2}}{\varepsilon \cosh s}\right)-\bar{Q}_{\varepsilon}^{\prime}\left(\frac{\tilde{w}+v_{1}}{\varepsilon \cosh s}\right)\right)\right\|_{0, \alpha, \delta} \leq c \varepsilon\left\|v_{2}-v_{1}\right\|_{2, \alpha, \delta}
\end{gathered}
$$

and finally

$$
\left\|\varepsilon \cosh s\left(\bar{Q}_{\varepsilon}^{\prime \prime}\left(\frac{\tilde{w}+v_{2}}{\varepsilon \cosh s}\right)-\bar{Q}_{\varepsilon}^{\prime \prime}\left(\frac{\tilde{w}+v_{1}}{\varepsilon \cosh s}\right)\right)\right\|_{0, \alpha, \delta} \leq c \varepsilon^{3 / 2}\left\|v_{2}-v_{1}\right\|_{2, \alpha, \delta} .
$$


We are using here that $\|\tilde{w} / \cosh s\|_{2, \alpha, 0} \leq c \varepsilon^{7 / 4}$ and $\left\|v_{i} / \cosh s\right\|_{2, \alpha, 0} \leq c \varepsilon^{9 / 4}$. The result follows at once for all $\varepsilon$ small enough.

To conclude this section we examine the Cauchy data map

$$
\mathcal{S}_{\varepsilon}:\left(\pi^{\prime \prime}\left(\mathcal{C}^{2, \alpha}\left(S^{1}\right)\right)\right)^{2} \longrightarrow\left(\mathcal{C}^{2, \alpha}\left(S^{1}\right) \times \mathcal{C}^{1, \alpha}\left(S^{1}\right)\right)^{2}
$$

given by

$$
\begin{aligned}
\mathcal{S}_{\varepsilon}\left(\phi_{ \pm}^{\prime \prime}\right)= & \left(\left(\varepsilon s_{\varepsilon}+\varepsilon \log (2 / \varepsilon)-w\left(s_{\varepsilon}, \cdot\right), \varepsilon-\partial_{s} w\left(s_{\varepsilon}, \cdot\right)\right)\right. \\
& \left(-\varepsilon s_{\varepsilon}+\varepsilon \log (2 / \varepsilon)+w\left(-s_{\varepsilon}, \cdot\right),-\varepsilon-\partial_{s} w\left(-s_{\varepsilon}, \cdot\right)\right)
\end{aligned}
$$

where $w$ is the solution of (31) given by Proposition 7 . We shall also consider the Cauchy data map $\mathcal{S}_{0}$ for the operator $\mathcal{P}_{s_{\varepsilon}}^{0}$. It is simple to check that

$$
\mathcal{S}_{0}\left(\phi_{ \pm}^{\prime \prime}\right)=\left(\left(\varepsilon s_{\varepsilon}+\varepsilon \log (2 / \varepsilon)-\phi_{+}^{\prime \prime}, \varepsilon-\left|D_{\theta}\right| \phi_{+}^{\prime \prime}\right),\left(-\varepsilon s_{\varepsilon}+\varepsilon \log (2 / \varepsilon)+\phi_{-}^{\prime \prime},-\varepsilon+\left|D_{\theta}\right| \phi_{-}^{\prime \prime}\right)\right) .
$$

The comparison between these two Cauchy data mappings plays a key role in our construction.

Corollary 1 The mappings $\mathcal{S}_{\varepsilon}$ and $\mathcal{S}_{0}$ are continuous. Furthermore, there exists a constant $c>0$ such that for any $\kappa>0$ there exists an $\varepsilon_{0}>0$ such that if $\varepsilon \in\left(0, \varepsilon_{0}\right]$, then for all $\left\|\phi_{ \pm}^{\prime \prime}\right\|_{2, \alpha} \leq \kappa \varepsilon^{3 / 2}$, we have

$$
\left\|\left(\mathcal{S}_{\varepsilon}-\mathcal{S}_{0}\right)\left(\phi_{ \pm}^{\prime \prime}\right)\right\|_{\left(\mathcal{C}^{2, \alpha} \times \mathcal{C}^{1, \alpha}\right)^{2}} \leq c \varepsilon^{3 / 2}
$$

Proof: The statement about continuity is straightforward and is left to the reader. Next, we must estimate the Cauchy data for the function $w-\mathcal{P}_{s_{\varepsilon}}^{0}\left(\phi_{ \pm}^{\prime \prime}\right)$. By Proposition 6, the Cauchy data of the function $\mathcal{P}_{s_{\varepsilon}}^{0}\left(\phi_{ \pm}^{\prime \prime}\right)$ differs from that of the function $w_{\varepsilon}=\mathcal{P}_{s_{\varepsilon}}\left(\phi_{ \pm}^{\prime \prime}\right)$ by a term of order $e^{-2 s_{\varepsilon}}\left\|\phi_{ \pm}^{\prime \prime}\right\|_{2, \alpha} \leq c \varepsilon^{2}$. Therefore we must estimate the Cauchy data of the function $w-w_{\varepsilon}=$ $\tilde{w}+v-w_{\varepsilon}$. Now it suffices to use (35) and the fact that $\|v\|_{2, \alpha, \delta} \leq 2 c_{\kappa} \varepsilon^{\delta / 4} \varepsilon^{2}$. This ends the proof of the Corollary.

It is important here that the constant $c$ is independent of $\kappa$. (See Definition 3 in $\S 8$ for the precise meaning with which this is meant to be understood.)

\section{The mean curvature operator for a graph}

Assume that $\Sigma \subset \mathbb{R}^{3}$ is a regular surface such that $0 \in \Sigma$ and $T_{0} \Sigma$ is the $x y$-plane. Then $\Sigma$ can be locally parameterized, near the origin, as a graph

$$
\mathbf{x}: B_{\bar{\rho}} \ni(x, y) \longrightarrow(x, y, u(x, y)) \in \Sigma \subset \mathbb{R}^{3},
$$

where $u: B_{\bar{\rho}} \longrightarrow \mathbb{R}$ is a regular function which satisfies

$$
\nabla^{k} u(x, y)=\mathcal{O}\left(r^{2-k}\right), \quad k \leq 2, \quad \nabla^{k} u(x, y)=\mathcal{O}(1), \quad k \geq 3,
$$

where $r=\left(x^{2}+y^{2}\right)^{1 / 2}$. In this parameterization, the mean curvature operator $H_{u}(x, y)$ of the graph defined by the function $u$ at the point of parameter $(x, y)$ is then given by [2]

$$
H_{u}(x, y)=\nabla\left(\frac{\nabla u}{\left(1+|\nabla u|^{2}\right)^{1 / 2}}\right) .
$$

Notice that we have defined the mean curvature to be the sum of the principal curvatures $H=$ $k_{1}+k_{2}$, not the average. 
By our assumptions, all surfaces sufficiently close to $\Sigma$ can be parameterized, in some neighborhood of 0 , as a vertical graph over a neighbourhood of 0 in the $x y$-plane, namely as

$$
B_{\rho} \ni(x, y) \longrightarrow(x, y, u(x, y)+w(x, y))
$$

for some (regular) function $w: B_{\rho} \longrightarrow \mathbb{R}$. by

It follows from (40) that the linearized mean curvature operator about $\Sigma$ is given explicitly

$$
\Lambda_{u}: w \longrightarrow \nabla\left(\frac{\nabla w}{\left(1+|\nabla u|^{2}\right)^{1 / 2}}-\frac{\nabla u \cdot \nabla w}{\left(1+|\nabla u|^{2}\right)^{3 / 2}} \nabla u\right)
$$

Performing the change of variable $(x, y)=e^{-t}(\cos \theta, \sin \theta)$, the linearized operator, which we still denote by $\Lambda_{u}$, has the form

$$
\Lambda_{u}: w \longrightarrow e^{2 t}\left(\partial_{t t}^{2}+\partial_{\theta \theta}^{2}\right)+\Lambda_{u}^{\prime},
$$

where $\Lambda_{u}^{\prime}$ is a second order partial differential operator with no terms of order zero and with coefficients bounded in $\mathcal{C}^{\infty}\left([-\log \rho,+\infty) \times S^{1}\right)$.

We also may expand the mean curvature $H_{u+w}$ of the surface parameterized by (41) in terms of the mean curvature $H_{u}$ of $\Sigma$ and $\Lambda_{u}$; thus

$$
H_{u+w}=H_{u}+\Lambda_{u} w-Q_{u}^{\prime}\left(e^{t} \nabla w, e^{t} \nabla^{2} w\right)-e^{t} Q_{u}^{\prime \prime}\left(e^{t} \nabla w, e^{t} \nabla^{2} w\right),
$$

where $Q_{u}^{\prime}$ and $Q_{u}^{\prime \prime}$ are functions with coefficients bounded in $\mathcal{C}^{\infty}\left([-\log \rho,+\infty) \times S^{1}\right)$ which satisfy

$$
Q_{u}^{\prime}(0,0)=Q_{u}^{\prime \prime}(0,0)=0 \quad \text { and } \quad \nabla Q_{u}^{\prime}(0,0)=\nabla Q_{u}^{\prime \prime}(0,0)=0 .
$$

and also

$$
\nabla^{2} Q_{u}^{\prime \prime}(0,0)=0
$$

These facts are established in Appendix II.

\section{Analytic modification of a surface $\Sigma_{0} \subset \mathbb{R}^{3}$ using Green's function}

Assume that $\Sigma_{0}$ is a regular, orientable CMC surface with boundary, positioned and oriented as in the previous section. We use a local chart $\mathbf{x}$ as in (38), with $\bar{\rho}<1$. We also assume that (39) holds in $B_{\bar{\rho}}$. We define, for $\rho \leq \bar{\rho} / 2$,

$$
\Sigma_{0}(\rho) \equiv \Sigma_{0} \backslash \mathbf{x}\left(B_{\rho}\right) .
$$

Still assuming that $\rho \leq \bar{\rho} / 2$, in $\Sigma_{0}$, we choose a unit vector field $\bar{\nu}$ which is equal to a normal unit vector field $\nu$ in $\Sigma_{0}(2 \rho)$ and which is equal to $(0,0,1)$ in $\mathbf{x}\left(B_{\rho}\right)$. We assume that $\nu \cdot \bar{\nu} \geq 1 / 2$ on all $\Sigma_{0}$. All surfaces near to $\Sigma_{0}$ can be parameterized by $\Sigma_{0} \ni p \longrightarrow p+w(p) \bar{\nu}(p)$ for some small function $w$. The linearized mean curvature operator

$$
\Lambda: \mathcal{C}_{\mathcal{D}}^{2, \alpha}\left(\Sigma_{0}\right)=\left\{w \in \mathcal{C}^{2, \alpha}\left(\Sigma_{0}\right): w=0 \quad \text { on } \quad \partial \Sigma_{0}\right\} \longmapsto \mathcal{C}^{0, \alpha}\left(\Sigma_{0}\right),
$$

relative to this vector field has the familiar form

$$
\Lambda \equiv \Delta_{\Sigma_{0}}+\left|\mathbf{A}_{\Sigma_{0}}\right|^{2}
$$

in $\Sigma_{0}(2 \rho)$, while in $\mathbf{x}\left(B_{\rho}\right)$ it is given by (42). Although not obvious at this stage, the use of $\bar{\nu}$ to parametrize nearby surfaces is intended to make the later analysis simpler.

By construction, $\Lambda$ depends on $\rho$. It follows from the analysis in Appendix I, cf. particularly (88), that $\Lambda$ tends to $\Delta_{\Sigma_{0}}+\left|\mathbf{A}_{\Sigma_{0}}\right|^{2}$ as $\rho \rightarrow 0$. In particular, if $\Sigma_{0}$ is nondegenerate in the sense 
of Definition 1, then $\Lambda$ is an isomorphism for $\rho$ sufficiently small. From now on, we shall assume that $\rho \leq \bar{\rho} / 2$ is fixed once for all so that this is the case. We may then solve the equation

$$
\Lambda \gamma_{0}=-2 \pi \delta_{0}, \quad \text { in } \quad \Sigma_{0},
$$

with $\gamma_{0}=0$ on $\partial \Sigma_{0}$.

The following Lemma follows easily from (42), using (39), and details are left to the reader.

Lemma 1 Assume that (39) holds and that $\gamma_{0}$ is the solution of (45). Then there exist constants $a_{0}, a_{1}, a_{2} \in \mathbb{R}$ such that, for all $k \geq 0$,

$$
\nabla^{k}\left(\gamma_{0}(x, y)-\bar{\gamma}_{0}(x, y)\right)=\mathcal{O}\left(r^{2-k} \log 1 / r\right),
$$

where $\bar{\gamma}_{0}(x, y)=-\log r+a_{0}+a_{1} x+a_{2} y$.

For $0<\varepsilon$ we define the surface $\bar{\Sigma}_{\varepsilon}$ to be the one parameterized by

$$
\Sigma_{0} \backslash B_{\varepsilon} \ni p \longrightarrow p+\varepsilon \gamma_{0}(p) \bar{\nu}(p) \in \mathbb{R}^{3} .
$$

If $\varepsilon$ is small enough, this is a regular surface.

We now compare the mean curvatures of $\bar{\Sigma}_{\varepsilon}$ and $\Sigma_{0}$.

Proposition 8 We may estimate the difference between $H_{\varepsilon}$, the mean curvature of $\bar{\Sigma}_{\varepsilon}$, and $H_{0}$, the mean curvature of $\Sigma_{0}$, by

$$
\nabla^{k}\left(H_{\varepsilon}-H_{0}\right)=\mathcal{O}\left(r^{-k}\left(\varepsilon^{2} r^{-2}+\varepsilon^{3} r^{-4}\right)\right) \quad \text { in } \quad \mathbf{x}\left(B_{\rho} \backslash B_{\varepsilon}\right)
$$

and by

$$
\nabla^{k}\left(H_{\varepsilon}-H_{0}\right)=\mathcal{O}\left(\varepsilon^{2}\right) \quad \text { in } \quad \Sigma_{0}(\rho)
$$

for all $k \geq 0$.

Proof : This follows at once from (44) with $w=\varepsilon \gamma_{0}$.

Corollary $2 H_{\varepsilon}$ is bounded independently of $\varepsilon$ in $\Sigma_{0} \backslash B_{c \varepsilon^{3 / 4}}$ for any fixed constant $c>0$.

Now, from Lemma 1, if $\bar{\Sigma}_{\varepsilon}$ is translated vertically (along the $z$-axis) by $-\varepsilon a_{0}$, then it will parameterized near 0 by

$$
\mathbf{x}_{\varepsilon}: \Sigma_{0} \backslash B_{\varepsilon} \ni(x, y) \longrightarrow(x, y,-\varepsilon \log r+u(x, y)+\mathcal{O}(\varepsilon r)) .
$$

Comparing this expansion with the one in (7) and using that $u(x, y)=\mathcal{O}\left(r^{2}\right)$, we see that the vertical distance between the two surfaces is estimated by $\mathcal{O}\left(r^{2}+\varepsilon r+\varepsilon^{3} r^{-2}\right)$. We have chosen the vertical translations of both the catenoid and $\Sigma_{0}$ carefully to minimize the distance between them. At any rate, this quantity is minimal for $r \sim \varepsilon^{3 / 4}$. This and Corollary 2 make it now quite reasonable that we restrict attention to a neighbourhood $r \geq c \varepsilon^{3 / 4}$, for any $c>0$. Thus we now define $\Sigma_{\varepsilon}$ to be the surface which is given near the origin by the parametrization

$$
\Sigma_{0}\left(c \varepsilon^{3 / 4}\right) \ni p \longrightarrow p+\varepsilon \gamma_{0}(p) \bar{\nu}(p) \in \mathbb{R}^{3} .
$$

The constant $0<c<1 / 8$ is now fixed once and for all. Notice that on $\Sigma_{\varepsilon}, \varepsilon r=\mathcal{O}\left(r^{2}\right)$. The 'inner boundary' of $\Sigma_{\varepsilon}$, created by the excised ball, will be denoted $\partial_{1} \Sigma_{\varepsilon}$. 


\section{Geometric modifications of the surface $\Sigma_{\varepsilon}$}

In order to match the Cauchy data of perturbations of the catenoid and of $\Sigma_{\varepsilon}$, it is necessary to allow some extra flexibility in the boundary data, specifically in the low $(j=0, \pm 1)$ eigenmodes. This flexibility is created by considering not just the surface $\Sigma_{\varepsilon}$, but also a family of modifications of it, comprised of rotations and translations and alterations of the parameter $\varepsilon$; the effect of these modifications in the boundary data is seen only in the low eigenmodes. In this section we define and analyze this family.

The parameter set for this family of surfaces will be denoted $\mathcal{A}=\left(\left(T_{1}, T_{2}, T_{3}\right),\left(R_{1}, R_{2}\right), e\right) \in \mathcal{U}$, where $\mathcal{U}$ is a neighbourhood of the origin in $\mathbb{R}^{3} \times \mathbb{R}^{2} \times \mathbb{R}$. The effect of the parameters $\left(T_{1}, T_{2}, T_{3}\right)$ will be to translate the surface by this vector. The parameters $\left(R_{1}, R_{2}\right)$ correspond to a rotation of the surface by the matrix

$$
\exp \left(\begin{array}{ccc}
0 & 0 & -R_{1} \\
0 & 0 & -R_{2} \\
R_{1} & R_{2} & 0
\end{array}\right)
$$

which has the form

$$
\left(\begin{array}{ccc}
1+\mathcal{O}\left(|R|^{2}\right) & \mathcal{O}\left(|R|^{2}\right) & -R_{1}+\mathcal{O}\left(|R|^{3}\right) \\
\mathcal{O}\left(|R|^{2}\right) & 1+\mathcal{O}\left(|R|^{2}\right) & -R_{2}+\mathcal{O}\left(|R|^{3}\right) \\
R_{1}+\mathcal{O}\left(|R|^{3}\right) & R_{2}+\mathcal{O}\left(|R|^{3}\right) & 1+\mathcal{O}\left(|R|^{2}\right)
\end{array}\right)
$$

Finally, $e$ changes the scaling parameter $\varepsilon$ into $\varepsilon-e$. Since these operations do not commute, we make the convention that $\mathcal{A}$ acts on $\Sigma_{\varepsilon}$ by first changing $\varepsilon$ to $\varepsilon-e$, next translating by $T_{3}$ in the vertical direction, then performing the rotation and finally translating by $\left(T_{1}, T_{2}\right)$ horizontally.

If the neighbourhood $\mathcal{U}$ is sufficiently small, the resulting surface, which will be denoted $\Sigma_{\varepsilon, \mathcal{A}}$, can still be locally parameterized as a graph over the $x y$-plane. We shall define a norm for the vector $\mathcal{A}$ by

$$
\|\mathcal{A}\| \equiv \varepsilon^{1 / 4}\left\|\left(T_{1}, T_{2}\right)\right\|_{\mathbb{R}^{2}}+|\log \varepsilon|^{-1}\left|T_{3}\right|+\varepsilon^{3 / 4}\left\|\left(R_{1}, R_{2}\right)\right\|_{\mathbb{R}^{2}}+|e| .
$$

This choice of scaling factors on the various components of $\mathcal{A}$ is necessitated by the analytic details of the ensuing arguments. In fact, this norm is related to the function $S^{1} \ni \theta \rightarrow\left(\xi(\theta), r \partial_{r} \xi(\theta)\right)$ for $r \sim \varepsilon^{3 / 4}$, where

$$
\xi: S^{1} \ni \theta \longrightarrow e \log r+T_{3}+r\left(R_{1} \cos \theta+R_{2} \sin \theta\right)+\varepsilon r^{-1}\left(T_{1} \cos \theta+T_{2} \sin \theta\right) .
$$

Hence it measures the effect of the geometric modifications on the set of points where the gluing will be done, see Proposition 10.

As we have already noted, in some neighbourhood of its inner boundary, $\Sigma_{\varepsilon, \mathcal{A}}$ can be written as a graph over the $x y$-plane, and this graph function can be compared to the graph function for the original surface $\Sigma_{0}$ and also to the one for the catenoidal (or rather, logarithmic) end. These 'comparison' graph functions will be denoted $w_{m}$ and $\hat{w}_{m}$, respectively. These functions depend on all the parameters. The main result of this section gives estimates on these functions, but first we introduce some convenient notation.

Definition 3 Henceforth the notation $f=\mathcal{O}(g(\varepsilon, r))$ shall mean that the function $f$ (usually on $\left.\Sigma_{\varepsilon, \mathcal{A}}\right)$ is bounded by a constant $c$ times the function $g$ of $\varepsilon$ and $r$, i.e. $f \leq c g(\varepsilon, r)$, where the constant $c$ does not depend on either $\varepsilon$ or $\kappa$. On the other hand, $f=\mathcal{O}_{\kappa}(g(\varepsilon, r))$ shall mean that $f$ is bounded similarly, but by a constant $c_{\kappa}$ which is allowed to depend on $\kappa$, but is still independent of $\varepsilon$. Furthermore, a bound of the latter type may sometimes by converted to a bound of the former type as follows. If, for example, $f=\mathcal{O}_{\kappa}\left(\varepsilon^{3 / 2}\right)$, and if we have (as shall always 
be true in all the calculations below) that the constant $c_{\kappa}$ in this estimate is bounded by a fixed polynomial in $\kappa$, then we also have $f=\mathcal{O}\left(\varepsilon^{3 / 4}\right)$, since $c_{\kappa} \varepsilon^{3 / 2} \leq c \varepsilon^{3 / 4}$ provided $\varepsilon$ is sufficiently small, for fixed $\kappa$. This reasoning will be justified because, although we need the flexibility to set $\kappa$ fairly large, once we have done so it will be fixed, and this will then determine an upper bound $\varepsilon_{0}$ for $\varepsilon$.

Proposition 9 Fix $\kappa>0$. Then there exists an $\varepsilon_{0}>0$ such that if $0<\varepsilon \leq \varepsilon_{0}$ and $\|\mathcal{A}\| \leq \kappa \varepsilon^{3 / 2}$, then for $\frac{1}{4} \varepsilon^{3 / 4} \leq r \leq \rho$, the surface $\Sigma_{\varepsilon, \mathcal{A}}$ can be parameterized as

1.

$$
(x, y) \longrightarrow\left(x, y, u(x, y)+w_{m}(x, y)\right),
$$

where $w_{m}(x, y)=\mathcal{O}_{\kappa}\left(\varepsilon^{3 / 4} r+\varepsilon|\log r|\right)$ and in addition, for all $k \geq 1, \nabla^{k} w_{m}(x, y)=$ $\mathcal{O}_{\kappa}\left(r^{-k}\left(\varepsilon^{3 / 4} r+\varepsilon\right)\right)$.

2.

$$
(x, y) \longrightarrow\left(x, y,-\varepsilon \log r+\hat{w}_{m}(x, y)\right),
$$

where $\hat{w}_{m}(x, y)=\mathcal{O}_{\kappa}\left(r^{2}+\varepsilon^{3 / 2}|\log \varepsilon|\right)$ and for $k \geq 1, \nabla^{k} \hat{w}_{m}(x, y)=\mathcal{O}_{\kappa}\left(r^{2-k}+\varepsilon^{3 / 2} r^{-k}\right)$.

Proof : We shall only prove the estimates for $w_{m}$ and $\hat{w}_{m}$ because the estimates for the derivatives follow from these in a straightforward manner.

First, notice that from $\|\mathcal{A}\| \leq \kappa \varepsilon^{3 / 2}$ we have

$$
\begin{array}{cl}
\left\|\left(T_{1}, T_{2}\right)\right\|_{\mathbb{R}^{2}} \leq \kappa \varepsilon^{5 / 4}, & \left|T_{3}\right| \leq \kappa \varepsilon^{3 / 2}|\log \varepsilon|, \\
|e| \leq \kappa \varepsilon^{3 / 2}, & \left\|\left(R_{1}, R_{2}\right)\right\|_{\mathbb{R}^{2}} \leq \kappa \varepsilon^{3 / 4} .
\end{array}
$$

We perform the transformation on all of $\mathbb{R}^{3}$, first translating vertically by $-e\left(\gamma_{0}-a_{0}\right)+T_{3}=$ $e \log r+T_{3}+\mathcal{O}(e r)$, then applying the rotation matrix and finally translating horizontally by the vector $T^{\prime}=\left(T_{1}, T_{2}\right)$. Acting on all of space, this effects a change from the coordinates $(x, y, z)$ to the coordinates $(\tilde{x}, \tilde{y}, \tilde{z})$. The precise relationship is

$$
\begin{aligned}
\tilde{x} & =T_{1}+\left(1+\mathcal{O}\left(|R|^{2}\right)\right) x+\mathcal{O}\left(|R|^{2}\right) y-\left(R_{1}+\mathcal{O}\left(|R|^{3}\right)\right)\left(e \log r+T_{3}+z+\mathcal{O}(e r)\right), \\
\tilde{y} & =T_{2}+\left(1+\mathcal{O}\left(|R|^{2}\right)\right) y+\mathcal{O}\left(|R|^{2}\right) x-\left(R_{2}+\mathcal{O}\left(|R|^{3}\right)\right)\left(e \log r+T_{3}+z+\mathcal{O}(e r)\right), \\
\tilde{z} & =\left(R_{1}+\mathcal{O}\left(|R|^{2}\right)\right) x+\left(R_{2}+\mathcal{O}\left(|R|^{2}\right) y+\left(1+\mathcal{O}\left(|R|^{2}\right)\right)\left(e \log r+T_{3}+z+\mathcal{O}(e r)\right) .\right.
\end{aligned}
$$

Recalling that $z=\varepsilon\left(\gamma_{0}(x, y)-a_{0}\right)+u(x, y)$, we first observe that

$$
|(\tilde{x}, \tilde{y})-(x, y)|=\mathcal{O}\left(|R|^{2} r+|R|\left(\varepsilon|\log r|+\left|T_{3}\right|+r^{2}+\varepsilon r\right)+\left|T^{\prime}\right|\right)=\mathcal{O}_{\kappa}\left(\varepsilon^{3 / 4} r^{2}+\varepsilon^{5 / 4}\right),
$$

for $\varepsilon$ sufficiently small. Hence if we set $\tilde{r}=|(\tilde{x}, \tilde{y})|$, we obtain

$$
\tilde{r}=r+\mathcal{O}_{\kappa}\left(\varepsilon^{3 / 4} r^{2}+\varepsilon^{5 / 4}\right),
$$

and in particular, we get for all $r \geq \varepsilon^{3 / 4} / 8$, we can state that $r / 2 \leq \tilde{r} \leq 2 r$ provided $\varepsilon$ is small enough. Using this first information, we obtain

$$
\log r=\log \tilde{r}+\mathcal{O}_{\kappa}\left(\varepsilon^{3 / 4} \tilde{r}+\varepsilon^{5 / 4} \tilde{r}^{-1}\right)
$$

and also

$$
r=\tilde{r}+\mathcal{O}_{\kappa}\left(\varepsilon^{3 / 4} \tilde{r}^{2}+\varepsilon^{5 / 4}\right)
$$

Inserting these estimates into the equations for $\tilde{x}$ and $\tilde{y}$ above, we see that

$$
(\tilde{x}-x, \tilde{y}-y)=T^{\prime}+\mathcal{O}_{\kappa}\left(\varepsilon^{7 / 4}|\log \tilde{r}|+\varepsilon^{3 / 4} \tilde{r}^{2}\right)=\mathcal{O}_{\kappa}\left(\varepsilon^{3 / 4} \tilde{r}^{2}+\varepsilon^{5 / 4}\right) .
$$


Thus, we can evaluate

$$
u(x, y)=u(\tilde{x}, \tilde{y})+\nabla u(\xi, \eta)(x-\tilde{x}, y-\tilde{y})+\mathcal{O}_{\kappa}\left(\varepsilon^{5 / 2}+\varepsilon^{3 / 2} \tilde{r}^{4}\right),
$$

where $(\xi, \eta)$ is some point on the line between $(x, y)$ and $(\tilde{x}, \tilde{y})$. Since $|(\xi, \eta)|=\mathcal{O}(r)$, we obtain $|\nabla u(\xi, \eta)|=\mathcal{O}(r)$, hence

$$
u(x, y)=u(\tilde{x}, \tilde{y})+\mathcal{O}_{\kappa}\left(\varepsilon^{3 / 4} \tilde{r}^{3}+\varepsilon^{5 / 4} \tilde{r}\right) .
$$

It is also an easy matter to check that

$$
\tilde{z}=z+\mathcal{O}_{\kappa}\left(\varepsilon^{3 / 4} \tilde{r}+\varepsilon^{3 / 2}|\log \varepsilon|\right) .
$$

and also that

$$
u(x, y)=u(\tilde{x}, \tilde{y})+\nabla u(\xi, \eta)(x-\tilde{x}, y-\tilde{y})+\mathcal{O}_{\kappa}\left(\varepsilon^{5 / 2}+\varepsilon^{3 / 2} \tilde{r}^{4}\right),
$$

where $(\xi, \eta)$ is some point on the line between $(x, y)$ and $(\tilde{x}, \tilde{y})$. Since $|(\xi, \eta)|=\mathcal{O}(r)$, we obtain $|\nabla u(\xi, \eta)|=\mathcal{O}(r)$. Finally, recalling again that $z=\varepsilon\left(\gamma_{0}(x, y)-a_{0}\right)+u(x, y)$, and collecting the previous estimates, we get

$$
\tilde{z}=u(\tilde{x}, \tilde{y})+\mathcal{O}_{\kappa}\left(\varepsilon^{3 / 4} \tilde{r}+\varepsilon|\log \tilde{r}|\right),
$$

which gives the desired estimate for $w_{m}$.

For the other part of the proposition, we wish to estimate the function $\hat{w}_{m}$, where

$$
\tilde{z}=-\varepsilon \log \tilde{r}+\hat{w}_{m}(\tilde{x}, \tilde{y}) .
$$

This time, we use the estimate $(52)$ to get

$$
\hat{w}_{m}=\mathcal{O}_{\kappa}\left(\tilde{r}^{2}+\varepsilon^{3 / 2}|\log \varepsilon|\right) .
$$

Corollary 3 The mean curvature $H_{\varepsilon, \mathcal{A}}$ of $\Sigma_{\varepsilon, \mathcal{A}}$ satisfies the same estimates as that of $\Sigma_{\varepsilon}$, namely

$$
\left|\nabla^{j}\left(H_{\varepsilon, \mathcal{A}}-H_{0}\right)\right|=\mathcal{O}\left(r^{-j}\left(\varepsilon^{2} r^{-2}+\varepsilon^{3} r^{-4}\right)\right) .
$$

Proof: Because $\Sigma_{\varepsilon, \mathcal{A}}$ is obtained from $\Sigma_{\varepsilon}$ by first modifying the Green function by an amount much less than $\varepsilon$ and then applying a rigid motion, it is clear that $H_{\varepsilon, \mathcal{A}}-H_{0}$ and all its derivatives are bounded by a multiple of $\varepsilon^{2}$ outside $B_{\bar{\rho}}$. Inside this ball we know that $\left|H_{\varepsilon, \mathcal{A}}-H_{0}\right|=$ $\mathcal{O}\left(\varepsilon^{2} r^{-2}+\varepsilon^{3} r^{-3}\right)$. From (53) one easily obtains

$$
\varepsilon^{2} r^{-2}+\varepsilon^{3} r^{-4}=\mathcal{O}\left(\varepsilon^{2} \tilde{r}^{-2}+\varepsilon^{3} \tilde{r}^{-4}\right),
$$

as desired. The bounds for the derivatives are similar.

We also require the following result.

Proposition 10 If $r \in\left[\frac{1}{4} \varepsilon^{3 / 4}, 4 \varepsilon^{3 / 4}\right]$, then the parameterization of $\Sigma_{\varepsilon, \mathcal{A}}$ has an expansion of the form

$$
(x, y) \longrightarrow\left(x, y,-\varepsilon \log r+w_{m}^{0}(x, y)+\bar{w}_{m}(x, y)\right),
$$

where

$$
w_{m}^{0}(x, y)=\left(e \log r+T_{3}+R_{1} x+R_{2} y+\varepsilon r^{-2}\left(T_{1} x+T_{2} y\right)\right)
$$

and, for all $k \geq 0,\left|\nabla^{k} \bar{w}_{m}(x, y)\right|=\mathcal{O}\left(\varepsilon^{(6-3 k) / 4}\right)$. 
Proof : We know, first of all, that

$$
(\tilde{x}-x, \tilde{y}-y)=T^{\prime}+\mathcal{O}_{\kappa}\left(\varepsilon^{7 / 4}|\log \varepsilon|\right)
$$

and then that

$$
u(x, y)=\mathcal{O}\left(\tilde{r}^{2}\right)=\mathcal{O}\left(\varepsilon^{3 / 2}\right) .
$$

In both of these we have used the upper bound on $r$. Finally,

$$
r^{2}=\tilde{r}^{2}-2\left(T_{1} \tilde{x}+T_{2} \tilde{y}\right)+\mathcal{O}_{\kappa}\left(\varepsilon^{5 / 2}|\log \varepsilon|\right),
$$

so that

$$
\log r=\log \tilde{r}-\frac{T_{1} \tilde{x}+T_{2} \tilde{y}}{\tilde{r}^{2}}+\mathcal{O}_{\kappa}(\varepsilon|\log \varepsilon|) .
$$

Putting these all together in the expression for $\tilde{z}$ yields

$$
\tilde{z}=-\varepsilon \log \tilde{r}+w_{m}^{0}(\tilde{x}, \tilde{y})+\mathcal{O}\left(\varepsilon^{3 / 2}\right)+\mathcal{O}_{\kappa}\left(\varepsilon^{7 / 4}\right),
$$

which gives the estimate for $\bar{w}_{m}$. The bounds for its derivatives are handled similarly.

\section{The linearized mean curvature operator about $\Sigma_{\varepsilon, \mathcal{A}}$}

In this section we shall study the Jacobi operator $\Lambda_{\varepsilon, \mathcal{A}}$ (relative to a transverse, but not everywhere normal unit vector field $\tilde{\nu}$ ) for the surface $\Sigma_{\varepsilon, \mathcal{A}}$. The results we obtain are the usual ones, namely solvability of $\Lambda_{\varepsilon, \mathcal{A}} u=f$ in appropriate weighted spaces with homogeneous Dirichlet boundary conditions (off the low eigenmodes) as well as of $\Lambda_{\varepsilon, \mathcal{A}} u=0$ with inhomogeneous Dirichlet boundary conditions.

It is slightly simpler to use a different parameterization now. Thus let $t=-\log r$ and set

$$
\bar{t}=-\log \rho+1>0 \quad \text { and } \quad t_{\varepsilon}=-\log (\varepsilon \cosh (1 / 4 \log \varepsilon)) .
$$

Notice that $t_{\varepsilon}=-3 / 4 \log \varepsilon+\log 2+\mathcal{O}\left(\varepsilon^{1 / 2}\right)$. The parametrization

$$
\tilde{\mathbf{x}}:(x, y) \longrightarrow\left(x, y, u(x, y)+w_{m}(x, y)\right),
$$

valid in $B_{\rho} \backslash B_{c \varepsilon^{3 / 4}}$ becomes

$$
\tilde{\mathbf{x}}:(t, \theta) \longrightarrow\left(e^{-t} \cos \theta, e^{-t} \sin \theta, u(t, \theta)+w_{m}(t, \theta)\right)
$$

for $(t, \theta) \in\left[\bar{t}-1,-\frac{3}{4} \log \varepsilon-\log c\right] \times S^{1}$. We have set here $u(t, \theta)=u\left(e^{-t} \cos \theta, e^{-t} \sin \theta\right)$ and $w_{m}(t, \theta)=w_{m}\left(e^{-t} \cos \theta, e^{-t} \sin \theta\right)$. We now define, for all $t \in\left[\bar{t}-1,-\frac{3}{4} \log \varepsilon-\log c\right)$

$$
\Sigma_{\varepsilon, \mathcal{A}}(t) \equiv \Sigma_{\varepsilon, \mathcal{A}} \backslash \tilde{\mathbf{x}}\left((t,-3 / 4 \log \varepsilon-\log c] \times S^{1}\right) .
$$

At this point we shall rename $\Sigma_{\varepsilon, \mathcal{A}} \equiv \Sigma_{\varepsilon, \mathcal{A}}\left(t_{\varepsilon}\right)$. Next, in $\Sigma_{\varepsilon, \mathcal{A}}$, we choose a unit vector field $\tilde{\nu}$ which is equal to a unit normal vector field $\nu$ in $\Sigma_{\varepsilon, \mathcal{A}}(\bar{t}-1)$ and which equals $(0,0,1)$ in $\tilde{\mathbf{x}}\left(\left[\bar{t}, t_{\varepsilon}\right) \times S^{1}\right)$. We assume that $\nu \cdot \tilde{\nu} \geq 1 / 2$, so that all surfaces near to $\Sigma_{\varepsilon, \mathcal{A}}$ are parameterized by $\Sigma_{\varepsilon, \mathcal{A}} \ni p \longrightarrow p+w(p) \tilde{\nu}(p)$, for a suitable function $w$. The linearized mean curvature operator, relative to $\tilde{\nu}$, is given by

$$
\Lambda_{\varepsilon, \mathcal{A}} \equiv \Delta_{\Sigma_{\varepsilon, \mathcal{A}}}+\left|\mathbf{A}_{\Sigma_{\varepsilon, \mathcal{A}}}\right|^{2} \quad \text { in } \quad \Sigma_{\varepsilon, \mathcal{A}}(\bar{t}-1),
$$

and by

$$
\Lambda_{\varepsilon, \mathcal{A}}=e^{2 t}\left(\partial_{t t}^{2}+\partial_{\theta \theta}^{2}\right)+\Lambda_{u}^{\prime}+\Lambda_{\varepsilon, \mathcal{A}}^{\prime},
$$


in $\left[\bar{t}-1, t_{\varepsilon}\right] \times S^{1}$. Here $\Lambda_{u}^{\prime}$ is the operator in (43)), and $\Lambda_{\varepsilon, \mathcal{A}}^{\prime}$ is the correction term coming from the geometric modifications, and in particular the extra term $w_{m}$ in the parametrization for $\Sigma_{\varepsilon, \mathcal{A}}$. It is a second order operator in $t$ and $\theta$, supported in $\left[\bar{t}-1, t_{\varepsilon}\right] \times S^{1}$, which may be calculated by differentiating (44) with respect to $w$ at $w=w_{m}$. To estimate its coefficients, we first note that the estimates for $w_{m}$ from Proposition 9 translate in the $(t, \theta)$ coefficients to

$$
w_{m}=\mathcal{O}_{\kappa}\left(\varepsilon^{3 / 4} e^{-t}+\varepsilon t\right) \quad \text { and } \quad\left|\nabla^{j} w_{m}\right|=\mathcal{O}_{\kappa}\left(\varepsilon^{3 / 4} e^{-t}+\varepsilon\right), \quad j \geq 1 .
$$

(To see this, recall that $\partial_{t}=-r \partial_{r}$.) Now it is not hard to check that the coefficients of $\Lambda_{\varepsilon, \mathcal{A}}^{\prime}$ and their derivatives are estimated by $\mathcal{O}_{\kappa}\left(\varepsilon^{3 / 4} e^{t}+\varepsilon e^{2 t}+\varepsilon^{2} e^{4 t}\right)$.

Before discussing the mapping properties of $\Lambda_{\varepsilon, \mathcal{A}}$, we define the weighted spaces on which we shall let it act.

Definition 4 For $k \in \mathbb{N}, 0<\alpha<1$ and $\delta \in \mathbb{R}$, define $\mathcal{C}_{\delta}^{k, \alpha}\left(\Sigma_{\varepsilon, \mathcal{A}}\right)$ by

$$
\left\{w \in \mathcal{C}_{\operatorname{loc}}^{k, \alpha}\left(\Sigma_{\varepsilon, \mathcal{A}}\right):\|w\|_{k, \alpha, \delta} \equiv\|w\|_{k, \alpha\left(\Sigma_{\varepsilon, \mathcal{A}}(\bar{t}+1)\right)}+\sup _{\bar{t} \leq t \leq t_{\varepsilon}-1} e^{-\delta t}|w \circ \tilde{\mathbf{x}}|_{k, \alpha,[t, t+1]}<\infty\right\}
$$

We may now state the main result of this section.

Proposition 11 Fix $\delta \in(1,2)$ and $\kappa>0$. Then for all $\varepsilon \in\left(0, \varepsilon_{0}\right]$ there exists an operator

$$
\Gamma_{\varepsilon, \mathcal{A}}: \mathcal{C}_{\delta+2}^{0, \alpha}\left(\Sigma_{\varepsilon, \mathcal{A}}\right) \longrightarrow \mathcal{C}_{\delta}^{2, \alpha}\left(\Sigma_{\varepsilon, \mathcal{A}}\right),
$$

such that if $f \in \mathcal{C}_{\delta+2}^{0, \alpha}\left(\Sigma_{\varepsilon, \mathcal{A}}\right)$, then $w=\Gamma_{\varepsilon, \mathcal{A}}(f)$ solves

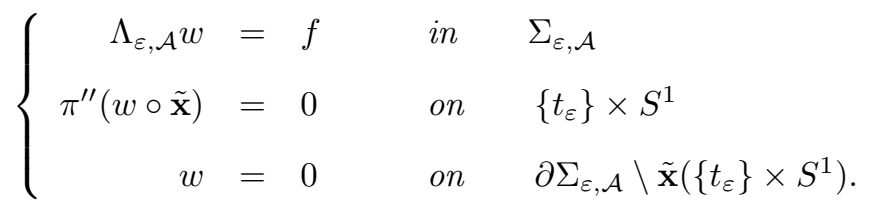

Furthermore, the norm of $\Gamma_{\varepsilon, \mathcal{A}}$ is bounded independently of $\varepsilon, \kappa$ and all $\mathcal{A}$ for which $\|\mathcal{A}\| \leq \kappa \varepsilon^{3 / 2}$.

Proof : The idea here is to construct a parametrix

$$
G: \mathcal{C}_{\delta+2}^{0, \alpha}\left(\Sigma_{\varepsilon, \mathcal{A}}\right) \longrightarrow \mathcal{C}_{\delta}^{2, \alpha}\left(\Sigma_{\varepsilon, \mathcal{A}}\right)
$$

whose norm is bounded by a constant $c>0$, provided $\varepsilon$ is small enough, by joining together two local parametrices. The first is constructed rather explicitly inside $B_{\rho}$, while the second, which acts on the exterior of this ball, is a cut-off of the solution operator for the Jacobi operator on all of $\Sigma_{0}$ (suitably translated and rotated by $\mathcal{A}$ ), which is known to exist by the nondegeneracy of this surface. The main point will be to show that the norm of $\Lambda_{\varepsilon, \mathcal{A}} \circ G-I$ can be made small, which immediately implies the result.

In this proof, $c$ will always denote a constant which does not depend on $\varepsilon, \kappa$ or $\mathcal{A}$, while $c_{\kappa}$ may depend on $\kappa$ but is independent of $\varepsilon$.

Appendix III contains some elementary results about the mapping properties of $e^{2 t}\left(\partial_{t t}^{2}+\partial_{\theta \theta}^{2}\right)$ on weighted spaces the cylinder $\left[\bar{t}, t_{\varepsilon}\right] \times S^{1}$ which we use now. First, from Lemma 1 there we obtain a right inverse for this operator with boundary conditions $w=0$ on $\bar{t} \times S^{1}$ and $\pi^{\prime \prime}(w)=0$ on $t_{\varepsilon} \times S^{1}$. Next, it is simple to check that

$$
\left\|\left(\Lambda_{u}^{\prime}+\Lambda_{\varepsilon, \mathcal{A}}^{\prime}\right) w\right\|_{0, \alpha, \delta+2} \leq\left(c e^{-2 \bar{t}}+c_{\kappa} \varepsilon^{1 / 2}\right)\|w\|_{2, \alpha, \delta} .
$$


From these two facts it is elementary to deduce the existence of a right inverse $G^{(i)}$ for $\Lambda_{\varepsilon, \mathcal{A}}=$ $e^{2 t} \Delta+\Lambda_{u}^{\prime}+\Lambda_{\varepsilon, \mathcal{A}}^{\prime}$ satisfying the appropriate boundary conditions and with norm bounded independently of $\varepsilon$, provided $\bar{t}$ is large enough. The superscript $(i)$ here is meant to connote that this is the parametrix inside the ball $B_{\rho}$.

Thus the operator $\lambda(\cdot-\bar{t}) G^{(i)}$ is well defined from $\mathcal{C}_{\delta+2}^{0, \alpha}\left(\Sigma_{\varepsilon, \mathcal{A}}\right)$ into $\mathcal{C}_{\delta}^{2, \alpha}\left(\Sigma_{\varepsilon, \mathcal{A}}\right)$ and has norm bounded uniformly in $\varepsilon$, for $\varepsilon$ small enough. Granted this, we see that the problem now reduces to solving (58) with $f$ replaced by $g \equiv f-\Lambda_{\varepsilon, \mathcal{A}}\left(\lambda G^{(i)}(f)\right)$. The key observation is that now $g$ has support in $\Sigma_{\varepsilon, \mathcal{A}}(\bar{t}+1)$, and in particular has a norm which is bounded by $c\|f\|_{0, \alpha, \delta+2}$ not only in the space $\mathcal{C}_{\delta+2}^{0, \alpha}\left(\Sigma_{\varepsilon, \mathcal{A}}\left(t_{\varepsilon}\right)\right)$ but also in $\mathcal{C}^{0, \alpha}\left(\Sigma_{\varepsilon, \mathcal{A}}\left(t_{\varepsilon}\right)\right)$.

To construct the other parametrix, which is an inverse for $\Lambda_{\varepsilon, \mathcal{A}}$ outside this ball, and which we shall denote by $G^{(o)}$, we first make the following construction. We modify the surface $\Sigma_{\varepsilon, \mathcal{A}}$ to one which has no boundary near zero by using the parametrization $(x, y) \rightarrow\left(x, y, u(x, y)+w_{m}(x, y)\right)$ and cutting off the function $w_{m}(x, y)$ in the region $\bar{t}+1 \leq t \leq \bar{t}+2$. More specifically, we let $\Sigma_{\varepsilon, \mathcal{A}}^{c}$ be the surface agreeing with $\Sigma_{\varepsilon, \mathcal{A}}$ outside $B_{\rho}$ and which is parametrized inside this ball by $(t, \theta) \rightarrow\left(e^{-t} \cos \theta, e^{-t} \sin \theta, u(t, \theta)+(1-\lambda(t-1-\bar{t})) w_{m}(t, \theta)\right)$. In $\Sigma_{\varepsilon, \mathcal{A}}^{c}$, we still choose a unit vector field $\hat{\nu}$ which is equal to the unit vector field $\tilde{\nu}$ in $\Sigma_{\varepsilon, \mathcal{A}}(\bar{t}+1)$ and which is equal to $(0,0,1)$ in the region $t \geq \bar{t}+2$. The bounds for the derivatives of $w_{m}$ show that the surfaces $\Sigma_{0}$ and $\Sigma_{\varepsilon, \mathcal{A}}^{c}$ are $\mathcal{C}^{2}$ close, and the Jacobi operator $\Lambda^{c}$ for $\Sigma_{\varepsilon, \mathcal{A}}^{c}$ differs from that for $\Sigma_{0}$ by terms of order $c_{\kappa} \varepsilon^{3 / 4}$. In particular, for $\varepsilon$ small enough, $\Lambda^{c}$ is also invertible from $\mathcal{C}^{2, \alpha}\left(\Sigma_{\varepsilon, \mathcal{A}}^{c}\right)$ into $\mathcal{C}^{0, \alpha}\left(\Sigma_{\varepsilon, \mathcal{A}}^{c}\right)$ (of course, respecting the Dirichlet boundary conditions at the boundary of $\Sigma_{\varepsilon, \mathcal{A}}^{c}$ ), and we let $G^{(o)}$ denote its inverse whose norm is bounded uniformly in $\varepsilon$.

We would like to have some information about the behavior of $G^{(o)}(g)$ near $0 \in \Sigma_{\varepsilon, \mathcal{A}}^{c}$ when $g$ has the form specified above and is extended by 0 . To this aim, we apply the result of Lemma 6 in Appendix III (for example with $\delta^{\prime}=\delta-2$ ). We find that there exist constants $J_{0}(f)$ (depending linearly on $f$ ) such that

$$
\left|J_{0}(f)\right|+\left\|G^{(o)}(g)-J_{0}(f)\right\|_{2, \alpha, \delta-2} \leq c_{\bar{t}}\|f\|_{0, \alpha, \delta+2} .
$$

We finally define

$$
G(f) \equiv J_{0}(f)+\lambda\left(t_{\varepsilon}-\cdot\right)\left(G^{(o)}(g)-J_{0}(f)\right)+\lambda(\cdot-\bar{t}) G^{(i)}(f),
$$

where $g \equiv f-\Lambda_{\varepsilon, \mathcal{A}}\left(\lambda G^{(i)}(f)\right)$ and where we are obviously setting $G^{(i)}=0$ for $t \leq \bar{t}$.

We also note that $\Lambda_{\varepsilon, \mathcal{A}}-\Lambda^{c}$ is an operator with coefficients which are $\mathcal{O}_{\kappa}\left(\varepsilon^{3 / 4} r^{-1}+\varepsilon r^{-2}+\right.$ $\left.\varepsilon^{2} r^{-4}\right)$ in the region $\bar{t} \leq t \leq t_{\varepsilon}$. Hence, it is easy to check that $\Lambda_{\varepsilon, \mathcal{A}} G=I+R$ where $R$ is a bounded operator on $\mathcal{C}_{\delta+2}^{0, \alpha}\left(\Sigma_{\varepsilon, \mathcal{A}}\right)$ with norm bounded by $c_{\kappa} \varepsilon^{3 / 4}$. As noted at the beginning, this suffices to complete the proof.

Following this same proof verbatim, but replacing Lemma 1 from Appendix III by Lemma and using Lemma 6 with $\delta^{\prime}=\delta-1$ instead of $\delta-2$, we also obtain

Proposition 12 Fix $\delta \in(0,1)$ and $\kappa>0$. Then for all $\varepsilon \in\left(0, \varepsilon_{0}\right]$ there exists an operator

$$
\hat{\Gamma}_{\varepsilon, \mathcal{A}}: \mathcal{C}_{\delta+2}^{0, \alpha}\left(\Sigma_{\varepsilon, \mathcal{A}}\right) \longrightarrow \mathcal{C}_{\delta}^{2, \alpha}\left(\Sigma_{\varepsilon, \mathcal{A}}\right),
$$

such that for all $f \in \mathcal{C}_{\delta+2}^{0, \alpha}\left(\Sigma_{\varepsilon, \mathcal{A}}\right)$, the function $w=\hat{\Gamma}_{\varepsilon, \mathcal{A}}(f)$ is a solution of the problem

$$
\left\{\begin{aligned}
\Lambda_{\varepsilon, \mathcal{A}} w & =f & & \text { in } & & \Sigma_{\varepsilon, \mathcal{A}} \\
\pi^{\prime}(w \circ \tilde{\mathbf{x}}) & =0 & & \text { on } & & \left\{t_{\varepsilon}\right\} \times S^{1} \\
w & =0 & & \text { on } & & \partial \Sigma_{\varepsilon, \mathcal{A}} \backslash \tilde{\mathbf{x}}\left(\left\{t_{\varepsilon}\right\} \times S^{1}\right) .
\end{aligned}\right.
$$

Furthermore, the norm of $\hat{\Gamma}_{t_{\varepsilon}, \mathcal{A}}$ is bounded independently of $\varepsilon, \kappa$ and all $\mathcal{A}$ for which $\|\mathcal{A}\| \leq \kappa \varepsilon^{3 / 2}$. 
In Lemma 7 of Appendix III we note the existence of the bounded operator

$$
\mathcal{P}: \pi^{\prime \prime}\left(\mathcal{C}^{2, \alpha}\left(S^{1}\right)\right) \longrightarrow \mathcal{C}_{-2}^{2, \alpha}\left((-\infty, 0] \times S^{1}\right)
$$

such that, for any $\phi^{\prime \prime} \in \pi^{\prime \prime}\left(\mathcal{C}^{2, \alpha}\left(S^{1}\right)\right), w=\mathcal{P}\left(\phi^{\prime \prime}\right)$ is the unique solution in $\mathcal{C}_{-2}^{2, \alpha}\left((-\infty, 0] \times S^{1}\right)$ of the problem

$$
\left\{\begin{aligned}
\Delta w & =0 & \text { in } & (-\infty, 0) \times S^{1} \\
w & =\phi^{\prime \prime} & \text { on } & \{0\} \times S^{1} .
\end{aligned}\right.
$$

Now define

$$
\Pi_{\varepsilon, \mathcal{A}}^{0}\left(\phi^{\prime \prime}\right) \circ \tilde{\mathbf{x}}(t, \theta) \equiv \lambda(t-\bar{t}) \mathcal{P}\left(\phi^{\prime \prime}\right)\left(t-t_{\varepsilon}, \theta\right) \quad \text { in } \quad\left[\bar{t}, t_{\varepsilon}\right] \times S^{1},
$$

and $\Pi_{\varepsilon, \mathcal{A}}^{0}\left(\phi^{\prime \prime}\right)=0$ in $\Sigma_{\varepsilon, \mathcal{A}}(\bar{t})$.

The counterpart of Proposition 6 is

Proposition 13 Fix $\delta \in(1,2)$ and $\kappa>0$. Then there exists an operator

$$
\Pi_{\varepsilon, \mathcal{A}}: \pi^{\prime \prime}\left(\mathcal{C}^{2, \alpha}\left(S^{1}\right)\right) \longrightarrow \mathcal{C}_{\delta}^{2, \alpha}\left(\Sigma_{\varepsilon, \mathcal{A}}\right),
$$

such that $w=\Pi_{\varepsilon, \mathcal{A}}\left(\phi^{\prime \prime}\right)$ satisfies

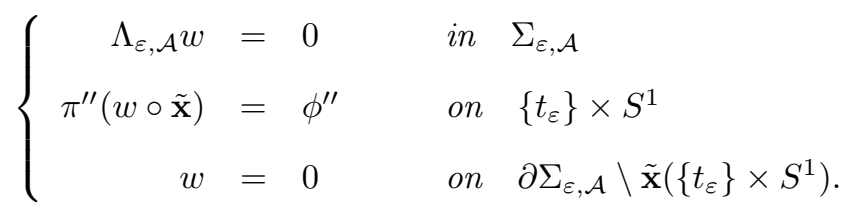

Furthermore, $\left\|\left(\Pi_{\varepsilon, \mathcal{A}}-\Pi_{\varepsilon, \mathcal{A}}^{0}\right)\left(\phi^{\prime \prime}\right)\right\|_{2, \alpha, \delta} \leq c_{\kappa}\left(\varepsilon^{3 / 2}+\varepsilon^{(3 \delta+2) / 4}\right)\left\|\phi^{\prime \prime}\right\|_{2, \alpha}$.

Proof: For simplicity, set $\tilde{w}(t, \theta)=\lambda(t-\bar{t}) \mathcal{P}\left(\phi^{\prime \prime}\right)\left(t-t_{\varepsilon}, \theta\right)$. The solution $w=\Pi_{\varepsilon, \mathcal{A}}\left(\phi^{\prime \prime}\right)$ is clearly given by $\tilde{w}-\Gamma_{\varepsilon, \mathcal{A}} \Lambda_{\varepsilon, \mathcal{A}} \tilde{w}$. It remains to estimate

$$
\|w-\tilde{w}\|_{2, \alpha, \delta} \leq c_{\kappa}\left\|\Lambda_{\varepsilon, \mathcal{A}} \tilde{w}\right\|_{0, \alpha, \delta+2} .
$$

For this we write $\tilde{w}=h\left(t-t_{\varepsilon}, \theta\right)$ in $\left[\bar{t}, t_{\varepsilon}\right] \times S^{1}$ and use

$$
\Lambda_{\varepsilon, \mathcal{A}} \tilde{w}=e^{2 t} \Delta \lambda \mathcal{P}\left(\phi^{\prime \prime}\right)\left(t-t_{\varepsilon}, \theta\right)+2 e^{2 t} \nabla \lambda \cdot \nabla \mathcal{P}\left(\phi^{\prime \prime}\right)\left(t-t_{\varepsilon}, \theta\right)+\left(\Lambda_{u}^{\prime}+\Lambda_{\varepsilon, \mathcal{A}}^{\prime}\right) \tilde{w} .
$$

Now, replacing $t-t_{\varepsilon}$ by $s \leq 0$, we see that

$$
\left\|\Lambda_{u}^{\prime} \tilde{w}\right\|_{0, \alpha, \delta+2} \leq c_{\kappa} e^{-(\delta+2) t_{\varepsilon}}\left\|\tilde{\Lambda}_{u}^{\prime} h(s)\right\|_{0, \alpha, \delta+2} \leq c_{\kappa} \varepsilon^{3(\delta+2) / 4}\left\|\phi^{\prime \prime}\right\|_{2, \alpha},
$$

where $\tilde{\Lambda}_{u}^{\prime}$ is the shift by $t_{\varepsilon}$ of $\Lambda_{u}^{\prime}$, and similarly, $\left\|\Lambda_{\varepsilon, \mathcal{A}} \tilde{w}\right\|_{0, \alpha, \delta+2}$ is estimated by the same quantity. Finally, the other two terms may be seen to be dominated by $c_{\kappa} \varepsilon^{3 / 2}|| \phi^{\prime \prime}||_{2, \alpha}$ because $|h(s)| \leq$ $c e^{-2 t_{\varepsilon}}\left\|\phi^{\prime \prime}\right\|_{2, \alpha}=c \varepsilon^{3 / 2}\left\|\phi^{\prime \prime}\right\|_{2, \alpha}$.

\section{CMC surfaces near $\Sigma_{\varepsilon, \mathcal{A}}$}

We maintain the notations of the last section. The surface parameterized by

$$
\Sigma_{\varepsilon, \mathcal{A}} \ni p \longrightarrow p+w(p) \tilde{\nu}(p)
$$

has mean curvature

$$
H=H_{\varepsilon, \mathcal{A}}+\Lambda_{\varepsilon, \mathcal{A}} w-Q_{\varepsilon, \mathcal{A}}(w)
$$


where $H_{\varepsilon, \mathcal{A}}$ is the mean curvature of $\Sigma_{\varepsilon, \mathcal{A}}$ and where $\mathcal{Q}_{\varepsilon, \mathcal{A}}(w)$ collects the nonlinear terms. The form of this nonlinear term near the origin is slightly different than before. Indeed, the uniformity of the coefficients in (44) specifically uses the fact that the expansion for $u$ does not have an $\varepsilon$ dependence. Thus we may not simply replace $u$ by $u+w_{m}$ there. Instead, we must replace $w$ by $w_{m}+w$ and then expand the terms around $w_{m}$, with the constant and linear terms in $w$ contributing to $H_{\varepsilon, \mathcal{A}}$ and $\Lambda_{\varepsilon, \mathcal{A}}$, respectively. One of the terms in the expansion of $Q_{u}^{\prime \prime}$ about $w_{m}$ is a quadratic term in $w$ with a coefficient of the form $\mathcal{O}\left(e^{2 t}\left(\left|\nabla w_{m}\right|+\left|\nabla^{2} w_{m}\right|\right)\right)$. Since $\left(\left|\nabla w_{m}\right|+\left|\nabla^{2} w_{m}\right|\right)=\mathcal{O}\left(\varepsilon^{3 / 4} e^{-t}+\varepsilon\right)$, we see that this coefficient is $\mathcal{O}\left(\varepsilon^{3 / 4} e^{t}+\varepsilon e^{2 t}\right)=\mathcal{O}\left(1+\varepsilon e^{2 t}\right)$. Hence altogether,

$$
\mathcal{Q}_{\varepsilon, \mathcal{A}}(w) \equiv\left(1+\varepsilon e^{2 t}\right) Q_{\varepsilon, \mathcal{A}}^{\prime}\left(e^{t} \nabla w, e^{t} \nabla^{2} w\right)+e^{t} Q_{\varepsilon, \mathcal{A}}^{\prime \prime}\left(e^{t} \nabla w, e^{t} \nabla^{2} w\right)
$$

where $Q_{\varepsilon, \mathcal{A}}^{\prime}$ and $Q_{\varepsilon, \mathcal{A}}^{\prime \prime}$ are quadratically and cubically vanishing functions with coefficients bounded in $\mathcal{C}^{k}\left(\left[\bar{t}, t_{\varepsilon}\right] \times S^{1}\right)$, for all $k \geq 0$, independently of $\kappa, \mathcal{A}$ and $\varepsilon$.

Given $\phi^{\prime \prime} \in \pi^{\prime \prime}\left(\mathcal{C}^{2, \alpha}\left(S^{1}\right)\right)$, we wish to construct a CMC surface which is a graph over $\Sigma_{\varepsilon, \mathcal{A}}$ and which has projected boundary values $\phi^{\prime \prime}$ on $\tilde{\mathbf{x}}\left(\left\{t_{\varepsilon}\right\} \times S^{1}\right)$. This is equivalent to solving the boundary value problem

$$
\left\{\begin{aligned}
\Lambda_{\varepsilon, \mathcal{A}} w & = & H_{0}-H_{\varepsilon, \mathcal{A}}+Q_{\varepsilon, \mathcal{A}}(w) & & \text { in } & \Sigma_{\varepsilon, \mathcal{A}} \\
\pi^{\prime \prime}\left(\left(u+w_{m}+w\right) \circ \tilde{\mathbf{x}}\right) & =\phi^{\prime \prime} & & \text { on } & & \left\{t_{\varepsilon}\right\} \times S^{1} \\
w & =0 & & \text { on } & & \partial \Sigma_{\varepsilon, \mathcal{A}} \backslash \tilde{\mathbf{x}}\left(\left\{t_{\varepsilon}\right\} \times S^{1}\right) .
\end{aligned}\right.
$$

Because we are using the modified normal vector field $\tilde{\nu}$, the surfaces we obtain will all have boundary which are vertical graphs over a fixed circle. Moreover, by our choice of $t_{\varepsilon}$ this circle is precisely the same one as we used for the catenoid.

Fixing $\kappa>0$, then for all $\phi^{\prime \prime} \in \pi^{\prime \prime}\left(\mathcal{C}^{2, \alpha}\left(S^{1}\right)\right)$ with $\left\|\phi^{\prime \prime}\right\|_{2, \alpha} \leq \kappa \varepsilon^{3 / 2}$, we define an approximation $\tilde{w}$ to the solution of $(63)$ by

$$
\tilde{w}=\Pi_{\varepsilon, \mathcal{A}}\left(\phi^{\prime \prime}-\pi^{\prime \prime}\left(\bar{w}_{m}\left(t_{\varepsilon}, \cdot\right)\right)+\hat{\Gamma}_{\varepsilon, \mathcal{A}}\left(H_{0}-H_{\varepsilon, \mathcal{A}}\right),\right.
$$

which is just a solution to (63) if the nonlinear term is set to zero. We are using the function $\bar{w}_{m}$ from Proposition 10 which satisfies, in particular, that $\pi^{\prime \prime}\left(u+w_{m}\right)=\pi^{\prime \prime} \bar{w}_{m}$ on $\tilde{\mathbf{x}}\left(\left\{t_{\varepsilon}\right\} \times S^{1}\right)$, but has the advantage that it is much smaller than $u+w_{m}$. We are also using the right inverse $\hat{\Gamma}_{\varepsilon, \mathcal{A}}$ from Proposition 12 here in the final term rather than the one from Proposition 11, which might be expected, simply because it affords us a better estimate, as we shall explain momentarily.

Before going on, we shall collect some estimates of $\tilde{w}$. Fix $\delta \in(1,2)$ as usual. First, let

$$
\tilde{w}_{0}=\Pi_{\varepsilon, \mathcal{A}}^{0}\left(\phi^{\prime \prime}-\pi^{\prime \prime}\left(\bar{w}_{m}\left(t_{\varepsilon}, \cdot\right)\right)\right) .
$$

We obtain from Proposition 13 that

$$
\left\|\tilde{w}_{0}\right\|_{2, \alpha, 2} \leq c \varepsilon^{3 / 2}\left\|\phi^{\prime \prime}-\pi^{\prime \prime}\left(\bar{w}_{m}\right)\right\|_{2, \alpha}
$$

and also

$$
\left\|\Pi_{\varepsilon, \mathcal{A}}\left(\phi^{\prime \prime}-\pi^{\prime \prime}\left(\bar{w}_{m}\right)\right)-\tilde{w}_{0}\right\|_{2, \alpha, \delta} \leq c_{\kappa}\left(\varepsilon^{3 / 2}+\varepsilon^{(3 \delta+2) / 4}\right)\left\|\phi^{\prime \prime}-\pi^{\prime \prime}\left(\bar{w}_{m}\right)\right\|_{2, \alpha} .
$$

Furthermore, from (55) in Proposition 9 we get

$$
\left\|\pi^{\prime \prime}\left(\bar{w}_{m}\right)\right\|_{2, \alpha} \leq c \varepsilon^{3 / 2} .
$$

Finally, the mean curvature $H_{\varepsilon, \mathcal{A}}$ is estimated in Corollary 3. Use this estimate and also applying Proposition 12 with $\delta=2 / 3$, we have

$$
\left\|\Gamma_{t_{\varepsilon}, \mathcal{A}}^{\prime}\left(H_{0}-H_{\varepsilon, \mathcal{A}}\right)\right\|_{2, \alpha, 2 / 3} \leq c \varepsilon^{2},
$$


for some constant $c>0$ which does not depend on $\kappa$.

Putting all of these estimates together, we obtain finally that

$$
\|\tilde{w}\|_{2, \alpha,[t, t+1]} \leq c\left(\varepsilon^{2} e^{2 t / 3}+\left(\varepsilon^{3}+\varepsilon^{(3 \delta+8) / 4}\right) e^{\delta t}+\varepsilon^{3} e^{2 t}\right) .
$$

The main reason we have had to use $\hat{\Gamma}_{\varepsilon, \mathcal{A}}$ rather than $\Gamma_{\varepsilon, \mathcal{A}}$ in $(68)$ is that otherwise the first term on the right in (69) would have a worse exponent, and this would lead to a far worse estimate in the next proposition.

Now let us solve (63). If we set $w=\tilde{w}+v$, then we must prove the existence of some $v \in \mathcal{C}_{\delta}^{2, \alpha}\left(\Sigma_{\varepsilon, \mathcal{A}}\right)$ such that

$$
\left\{\begin{aligned}
\Lambda_{\varepsilon, \mathcal{A}} v & =\mathcal{Q}_{\varepsilon, \mathcal{A}}(\tilde{w}+v) & & \text { in } & & \Sigma_{\varepsilon, \mathcal{A}} \\
\pi^{\prime \prime}(v \circ \tilde{\mathbf{x}}) & =0 & & \text { on } & & \left\{t_{\varepsilon}\right\} \times S^{1} \\
v & =0 & & \text { on } & & \partial \Sigma_{\varepsilon, \mathcal{A}} \backslash \tilde{\mathbf{x}}\left(\left\{t_{\varepsilon}\right\} \times S^{1}\right) .
\end{aligned}\right.
$$

As before, it is enough to find a fixed point of the mapping

$$
\mathcal{M}_{\varepsilon, \mathcal{A}}(v)=\Gamma_{\varepsilon, \mathcal{A}}\left(\mathcal{Q}_{\varepsilon, \mathcal{A}}(\tilde{w}+v)\right) .
$$

Proposition 14 For any $\kappa>0$, there exist $c_{\kappa}>0$ and $\varepsilon_{0}>0$ such that if $\varepsilon \in\left(0, \varepsilon_{0}\right]$ and $\left\|\phi^{\prime \prime}\right\|_{2, \alpha} \leq \kappa \varepsilon^{3 / 2}$, then

$$
\left\|\mathcal{M}_{\varepsilon, \mathcal{A}}(0)\right\|_{2, \alpha, \delta} \leq c_{\kappa} \varepsilon^{(10+3 \delta) / 4}
$$

and

$$
\left\|\mathcal{M}_{\varepsilon, \mathcal{A}}\left(v_{2}\right)-\mathcal{M}_{\varepsilon, \mathcal{A}}\left(v_{1}\right)\right\|_{2, \alpha, \delta} \leq \frac{1}{2}\left\|v_{2}-v_{1}\right\|_{2, \alpha, \delta},
$$

provided $v_{1}$ and $v_{2}$ belong to $B \equiv\left\{v:\|v\|_{2, \alpha, \delta} \leq c_{\kappa} \varepsilon^{(10+3 \delta) / 4}\right\}$. In particular, the mapping $\mathcal{M}_{\varepsilon, \mathcal{A}}$ is a contraction on the ball $B$ into itself and thus $\mathcal{M}_{\varepsilon, \mathcal{A}}$ has a unique fixed point $v$ in this ball.

Proof: The proof is nearly identical to the proof of Proposition 7. The first thing we must establish is that $e^{t} \nabla^{j} \tilde{w}$ is bounded, and small, so that we may estimate $Q_{\varepsilon, \mathcal{A}}^{\prime}\left(e^{t} \nabla \tilde{w}, e^{t} \nabla^{2} \tilde{w}\right)$ by $e^{2 t}\left(|\nabla \tilde{w}|^{2}+\left|\nabla^{2} \tilde{w}\right|^{2}\right)$, for example, and similarly for the other nonlinear term. If we call the function of $t$ on the right side of (69) $h(t)$, say, then we observe that it is convex, and

$$
h(\bar{t}) \leq c \varepsilon^{2}, \quad h\left(t_{\varepsilon}\right) \leq c \varepsilon^{3 / 2} .
$$

Hence $e^{t}\left|\nabla^{j} \tilde{w}\right| \leq \varepsilon^{3 / 4}, j=1,2$, as desired.

Now,

$$
\left\|\mathcal{M}_{\varepsilon, \mathcal{A}}(0)\right\|_{2, \alpha, \delta} \leq c\left\|Q_{\varepsilon, \mathcal{A}}(\tilde{w})\right\|_{0, \alpha, \delta+2}
$$

which is estimated by the supremum of

$$
e^{-(\delta+2) t}\left(\left(1+\varepsilon e^{2 t}\right) e^{2 t} h(t)^{2}+e^{4 t} h(t)^{3}\right)=h(t)^{2} e^{-\delta t}\left(\left(1+\varepsilon e^{2 t}\right)+e^{2 t} h(t)\right) .
$$

Checking the values at $t=\bar{t}$ and $t=t_{\varepsilon}$ and using that the value at $\bar{t}$ also dominates the behaviour in all of $\Sigma_{\varepsilon, \mathcal{A}}(\bar{t})$, we see that

$$
\left\|\mathcal{M}_{\varepsilon, \mathcal{A}}(0)\right\|_{2, \alpha, \delta} \leq c \varepsilon^{(10+3 \delta) / 4} .
$$

This completes the proof of the first estimate. The second one is similar and left to the reader.

We conclude this section with the counterpart of Corollary 1. As we have already mentioned, we have defined $t_{\varepsilon}$ in such a way that the (Dirichlet) boundary data of the surfaces defined by 
Proposition 7 and Proposition 15 are curves on the same cylinder. In the next section we shall compare the Neumann data of the solutions of (29) and (63), and naturally we must differentiate with respect to the same normal. To this aim, we note that the relationship between the $s$ and $t$ variables on the catenoid and surface $\Sigma_{0}$ is given by $e^{-t}=\varepsilon \cosh s$ (where we assume that $t$ is close to $t_{\varepsilon}$ and $s$ is close to $\left.s_{\varepsilon}\right)$. Differentiating this at $t=t_{\varepsilon}, s=s_{\varepsilon}$ gives $(d t / d s)\left(s_{\varepsilon}\right)=\tanh s_{\varepsilon}$. Since $s_{\varepsilon}=-(1 / 4) \log \varepsilon$,

$$
\tanh s_{\varepsilon} \equiv \eta_{\varepsilon}=\frac{1-\varepsilon^{1 / 2}}{1+\varepsilon^{1 / 2}}
$$

We also recall the function $w_{m}^{0}$ from Proposition 10; in terms of the $(t, \theta)$ coordinates,

$$
w_{m}^{0}(t, \theta) \equiv e t+T_{3}+e^{-t}\left(R_{1} \cos \theta+R_{2} \sin \theta\right)+\varepsilon e^{t}\left(T_{1} \cos \theta+T_{2} \sin \theta\right) .
$$

Recalling also the neighbourhood $\mathcal{U}$ where the parameters $\mathcal{A}$ reside, we set

$$
\mathcal{F} \equiv \mathcal{U} \times \pi^{\prime \prime}\left(\mathcal{C}^{2, \alpha}\left(S^{1}\right)\right),
$$

endowed with the norm

$$
\|(\mathcal{A}, w)\|_{\mathcal{F}} \equiv\|\mathcal{A}\|+\|w\|_{2, \alpha} .
$$

We now define the (slightly modified) Cauchy data mappings $\mathcal{T}_{\mathcal{\varepsilon}}$ for the CMC problem over $\Sigma_{\varepsilon, \mathcal{A}}$ and $\mathcal{T}_{0}$ for the Laplacian on the half-cylinder $\left(-\infty, t_{\varepsilon}\right) \times S^{1}$ :

Definition 5 For $\phi^{\prime \prime} \in \pi^{\prime \prime}\left(\mathcal{C}^{2, \alpha}\left(S^{1}\right)\right)$ with $\left\|\phi^{\prime \prime}\right\|_{2, \alpha} \leq \kappa \varepsilon^{3 / 2}$, let $w=\tilde{w}+v$ be the solution of (70) given by Proposition 14. Then we define

$$
\begin{gathered}
\mathcal{T}_{\varepsilon}: \mathcal{F} \longrightarrow \mathcal{C}^{2, \alpha}\left(S^{1}\right) \times \mathcal{C}^{1, \alpha}\left(S^{1}\right) \\
\left(\mathcal{A}, \phi^{\prime \prime}\right) \longmapsto\left(\varepsilon t_{\varepsilon}+w_{m}^{0}\left(t_{\varepsilon}, .\right)+\left(\bar{w}_{m}+w\right)\left(t_{\varepsilon}, .\right),-\eta_{\varepsilon}\left(\varepsilon+\partial_{t} w_{m}^{0}\left(t_{\varepsilon}, .\right)+\partial_{t}\left(\bar{w}_{m}+w\right)\left(t_{\varepsilon}, .\right)\right)\right)
\end{gathered}
$$

and

$$
\begin{gathered}
\mathcal{T}_{0}: \mathcal{F} \longrightarrow \mathcal{C}^{2, \alpha}\left(S^{1}\right) \times \mathcal{C}^{1, \alpha}\left(S^{1}\right) \\
\left(\mathcal{A}, \phi^{\prime \prime}\right) \longmapsto\left(\varepsilon t_{\varepsilon}+w_{m}^{0}\left(t_{\varepsilon}, .\right)+\phi^{\prime \prime},-\eta_{\varepsilon}\left(\varepsilon+\partial_{t} w_{m}^{0}+\left|D_{\theta}\right| \phi^{\prime \prime}\right)\right) .
\end{gathered}
$$

We have made two modifications which are worth pointing out. First, the factor $\eta_{\varepsilon}$ is included so as to correspond with differentiation with respect to $s$ on the catenoid. Second, this is the Cauchy data with respect to the inward pointing normal, because we are using the outward pointing normal on the catenoid.

Corollary 4 For any $\kappa>0$ there exists an $\varepsilon_{0}>0$ and a constant $c>0$ independent of $\kappa$ such that if $\varepsilon \in\left(0, \varepsilon_{0}\right]$, then $\mathcal{T}_{\varepsilon}$ and $\mathcal{T}_{0}$ are continuous and satisfy

$$
\left\|\left(\mathcal{T}_{\varepsilon}-\mathcal{T}_{0}\right)\left(\mathcal{A}, \phi^{\prime \prime}\right)\right\|_{\mathcal{C}^{2, \alpha} \times \mathcal{C}^{1, \alpha}} \leq c \varepsilon^{3 / 2} .
$$

Proof: The proof is essentially identical to the one for Corollary 11. Continuity of the operators is obvious. We decompose

$$
\begin{gathered}
w_{m}+w=\varepsilon t+w_{m}^{0}+\bar{w}_{m}+\tilde{w}+v \\
=\varepsilon t+w_{m}^{0}+\bar{w}_{m}+\Pi_{\varepsilon, \mathcal{A}}\left(\phi^{\prime \prime}\right)-\Pi_{\varepsilon, \mathcal{A}}\left(\pi^{\prime \prime}\left(\bar{w}_{m}\left(t_{\varepsilon}, \cdot\right)\right)\right)+\hat{\Gamma}_{\varepsilon, \mathcal{A}}\left(1-H_{\varepsilon, \mathcal{A}}\right)+v .
\end{gathered}
$$

The (cut off) harmonic function on the cylinder for which $\mathcal{T}_{0}$ is the Cauchy data operator is

$$
\varepsilon t+w_{m}^{0}+\Pi_{\varepsilon, \mathcal{A}}^{0}\left(\phi^{\prime \prime}\right) .
$$

Hence

$$
\left(\mathcal{T}_{\varepsilon}-\mathcal{T}_{0}\right)\left(\mathcal{A}, \phi^{\prime \prime}\right)=\bar{w}_{m}+\left(\Pi_{\varepsilon, \mathcal{A}}-\Pi_{\varepsilon, \mathcal{A}}^{0}\right)\left(\phi^{\prime \prime}\right)-\Pi_{\varepsilon, \mathcal{A}}\left(\pi^{\prime \prime}\left(\bar{w}_{m}\left(t_{\varepsilon}, \cdot\right)\right)\right)+\hat{\Gamma}_{\varepsilon, \mathcal{A}}\left(1-H_{\varepsilon, \mathcal{A}}\right)+v .
$$

We estimate these in turn using Propositions 10, 13, equation (68) and finally Proposition 14 to obtain the final estimate. 


\section{Application to our problem}

Let us now return to our original geometric problem. We are given two CMC surfaces $\Sigma_{1}$ and $\Sigma_{2}$ which satisfy the assumptions of Theorem 11. In this section we outline the (very) minor changes that are needed to apply the preceding results in our context.

First, the results of section 5 may be applied directly to the truncated rescaled catenoid. Similarly, we may directly apply the results of sections 7,8 , and 10 to the surface $\Sigma_{1}$. In particular, we obtain the corresponding mappings $\mathcal{T}_{\varepsilon}$ and $\mathcal{T}_{0}$, which we shall denote by $\mathcal{T}_{\varepsilon}^{-}$and $\mathcal{T}_{0}^{-}$, respectively. This superscript is meant to imply that $\Sigma_{1}$ is the surface lying 'underneath' $\Sigma_{2}$, and that its oriented normal at the origin is $(0,0,1)$.

However, $\Sigma_{2}$ is oriented oppositely, so that its normal at the origin is $(0,0,-1)$. Thus, in section 4 , the vector field $\bar{\nu}$ now should equal $(0,0,-1)$ in $\mathbf{x}\left(B_{\rho}\right)$. The analytic modification, by adding $\varepsilon$ times the Green function on $\Sigma_{2}$, and then translating vertically by $\varepsilon a_{0}$, proceeds exactly as before. The geometric modifications of section 8 also proceed as before. However, recall from section 3 that we had translated the catenoid vertically by the amount $\varepsilon \log (2 / \varepsilon)$, so that its match with $\Sigma_{1}$ would be optimal. To make such a match with $\Sigma_{2}$ at its upper boundary, we can not, of course, translate the catenoid again, so instead we translate $\Sigma_{2}$ vertically by the amount $V_{\varepsilon}=2 \varepsilon \log (2 / \varepsilon)$. The result is that the analogues of (50), (51) and (55) are

$$
\begin{gathered}
(x, y) \longrightarrow\left(x, y, V_{\varepsilon}+u_{2}(x, y)-w_{m}(x, y)\right), \\
(x, y) \longrightarrow\left(x, y, V_{\varepsilon}+\varepsilon \log r-\hat{w}_{m}(x, y)\right),
\end{gathered}
$$

and

$$
(x, y) \longrightarrow\left(x, y, V_{\varepsilon}+\varepsilon \log r-w_{m}^{0}-\bar{w}_{m}(x, y)\right),
$$

respectively, where $u_{2}$ is the graph function for $\Sigma_{2}$ and where the functions $w_{m}, \hat{w}_{m}, w_{m}^{0}$ and $\bar{w}_{m}$ are the direct analogues of the corresponding functions for $\Sigma_{1}$. We shall let the functions $w_{m}^{0}$ corresponding to the two surfaces be denoted $\left(w_{m}^{0}\right)_{ \pm}$, respectively. The other functions will not need to be so explicitly labeled.

The vector field $\tilde{\nu}$ in section 9 now equals $(0,0,-1)$ in $\tilde{\mathbf{x}}\left(\left[\bar{t}, t_{\varepsilon}\right] \times S^{1}\right)$, but this section remains unchanged otherwise. Finally, in section 10, the Cauchy data operators become

$$
\begin{gathered}
\mathcal{T}_{\varepsilon}^{+}: \mathcal{F} \longrightarrow \mathcal{C}^{2, \alpha}\left(S^{1}\right) \times \mathcal{C}^{1, \alpha}\left(S^{1}\right) \\
\left(\mathcal{A}, \phi^{\prime \prime}\right) \longmapsto\left(V_{\varepsilon}-\left(\left(w_{m}^{0}\right)_{+}+w_{m}+w\right)\left(t_{\varepsilon}, \cdot\right), \eta_{\varepsilon}\left(\varepsilon+\partial_{t}\left(w_{m}^{0}\right)_{+}\left(t_{\varepsilon}, \cdot\right)+\partial_{t}\left(w_{m}+w\right)\left(t_{\varepsilon}, \cdot\right)\right)\right)
\end{gathered}
$$

and

$$
\begin{gathered}
\mathcal{T}_{0}^{+}: \mathcal{F} \longrightarrow \mathcal{C}^{2, \alpha}\left(S^{1}\right) \times \mathcal{C}^{1, \alpha}\left(S^{1}\right) \\
\left(\mathcal{A}, \phi^{\prime \prime}\right) \longmapsto\left(V_{\varepsilon}-\left(w_{m}^{0}\right)_{+}\left(t_{\varepsilon}, \cdot\right)-\phi^{\prime \prime}, \eta_{\varepsilon}\left(\varepsilon+\partial_{t}\left(w_{m}^{0}\right)_{+}\left(t_{\varepsilon}, \cdot\right)+\left|D_{\theta}\right| \phi^{\prime \prime}\right)\right) .
\end{gathered}
$$

\section{Matching the Cauchy data}

We will denote by $\mathcal{B}_{\kappa}^{\prime}$ and $\mathcal{B}_{\kappa}^{\prime \prime}$ the balls of radius $\kappa \varepsilon^{3 / 2}$ in the parameter space $\mathcal{U}$ for $\mathcal{A}$ and in $\pi^{\prime \prime}\left(\mathcal{C}^{2, \alpha}\left(S^{1}\right)\right)$, respectively. The product $\mathcal{B}_{\kappa}^{\prime} \times \mathcal{B}_{\kappa}^{\prime \prime}$ will be denoted simply $\mathcal{B}_{\kappa}$. All of the constructions in the previous sections are valid for $\left(\mathcal{A}, \phi^{\prime \prime}\right) \equiv\left(\mathcal{A}_{ \pm}, \phi_{ \pm}^{\prime \prime}\right) \in \mathcal{B}_{\kappa}^{2}$ for any fixed $\kappa>0$, provided $\varepsilon$ is sufficiently small.

We now define the difference of the Cauchy data operators:

$$
\begin{gathered}
\mathbf{C}_{\varepsilon}: \mathcal{B}_{\kappa}^{2} \longrightarrow\left(\mathcal{C}^{2, \alpha}\left(S^{1}\right) \times \mathcal{C}^{1, \alpha}\left(S^{1}\right)\right)^{2} \\
\left(\mathcal{A}, \phi^{\prime \prime}\right) \longmapsto\left(\left(\mathcal{T}_{\varepsilon}^{+}\left(\mathcal{A}_{+}, \phi_{+}^{\prime \prime}\right)-\mathcal{S}_{\varepsilon}\left(\phi_{ \pm}^{\prime \prime}\right)_{+}\right),\left(\mathcal{T}_{\varepsilon}^{-}\left(\mathcal{A}_{-}, \phi_{-}^{\prime \prime}\right)-\mathcal{S}_{\varepsilon}\left(\phi_{ \pm}^{\prime \prime}\right)_{-}\right)\right),
\end{gathered}
$$


where we have denoted by $\mathcal{S}_{\varepsilon}\left(\phi_{ \pm}^{\prime \prime}\right)_{ \pm}$the component of $\mathcal{S}_{\varepsilon}\left(\phi_{ \pm}^{\prime \prime}\right)$ at the upper and lower boundaries, respectively. Setting

$$
\mathcal{E}=\operatorname{Span}\{1, \cos \theta, \sin \theta\}
$$

then by construction,

$$
\text { range } \mathbf{C}_{\varepsilon} \subset\left(\mathcal{E} \times \mathcal{C}^{1, \alpha}\left(S^{1}\right)\right)^{2} \text {. }
$$

Proposition 15 There exists a $\kappa_{0}>0$ such that if $\kappa>\kappa_{0}$ then there is an $\varepsilon_{0}>0$ for which, if $0<\varepsilon<\varepsilon_{0}$, then $\mathbf{C}_{\varepsilon}$ has a unique zero in $\mathcal{B}_{\kappa}^{2}$.

This Proposition produces a CMC surface $S_{\varepsilon}$ for each admissible $\varepsilon$. Indeed, if $\mathbf{C}_{\varepsilon}\left(\mathcal{A}, \phi^{\prime \prime}\right)=0$, then there are smooth CMC surfaces $\Sigma_{1}\left(\mathcal{A}_{-}, \phi_{-}^{\prime \prime}\right), \Sigma_{2}\left(\mathcal{A}_{+}, \phi_{+}^{\prime \prime}\right)$ and the CMC perturbation of the truncated rescaled catenoid which we denote by $\left.\mathcal{C}_{\varepsilon}^{c}\left(\phi_{ \pm}^{\prime \prime}\right)\right)$, the union of which match up to be $\mathcal{C}^{1}$ across the two curves. Because of the elliptic nature of the CMC equation, it is standard that this union is actually $\mathcal{C}^{\infty}$ across these curves, and hence $S_{\varepsilon}$ is a regular CMC surface.

Thus, in order to complete the proof of Theorem 1, it remains to prove the Proposition 15.

Proof : Let us set

$$
\begin{gathered}
\mathbf{C}_{0}: \mathcal{B}_{\kappa}^{2} \longrightarrow\left(\mathcal{C}^{2, \alpha}\left(S^{1}\right) \times \mathcal{C}^{1, \alpha}\left(S^{1}\right)\right)^{2} \\
\left(\mathcal{A}, \phi^{\prime \prime}\right) \longmapsto\left(\left(\mathcal{T}_{0}^{+}\left(\mathcal{A}_{+}, \phi_{+}^{\prime \prime}\right)-\mathcal{S}_{0}\left(\phi_{ \pm}\right)_{+}\right),\left(\mathcal{T}_{0}^{-}\left(\mathcal{A}_{-}, \phi_{-}^{\prime \prime}\right)-\mathcal{S}_{0}\left(\phi_{ \pm}\right)_{-}\right)\right) .
\end{gathered}
$$

From Corollaries 1 and 1 , we obtain

$$
\left\|\left(\mathbf{C}_{\varepsilon}-\mathbf{C}_{0}\right)\left(\mathcal{A}, \phi^{\prime \prime}\right)\right\|_{\left(\mathcal{C}^{2, \alpha} \times \mathcal{C}^{1, \alpha}\right)^{2}} \leq c \varepsilon^{3 / 2},
$$

where the constant $c>0$ does not depend on $\kappa$.

We examine the map $\mathbf{C}_{0}$ more closely. Recall first the small deviation, of order $\mathcal{O}(\varepsilon r)$ of the translated catenoid from $-\varepsilon \log r$. This error term is clearly radial, since the catenoid is rotationally symmetric, and hence we write it as $\varepsilon \beta(r)$, where $\beta(0)=0$. Now

$$
\begin{aligned}
\mathbf{C}_{0}\left(\mathcal{A}, \phi^{\prime \prime}\right)= & \left(\left(\left(w_{m}^{0}\right)_{+}+\varepsilon \beta(r), \eta_{\varepsilon}\left(\partial_{t}\left(\left(w_{m}^{0}\right)_{+}+\varepsilon \beta(r)\right)\right)-\left(\eta_{\varepsilon}-1\right)\left|D_{\theta}\right| \phi_{+}^{\prime \prime}\right),\right. \\
& \left.\left(\left(w_{m}^{0}\right)_{-}-\varepsilon \beta(r), \eta_{\varepsilon}\left(\partial_{t}\left(\left(w_{m}^{0}\right)_{-}+\varepsilon \beta(r)\right)\right)+\left(\eta_{\varepsilon}-1\right)\left|D_{\theta}\right| \phi_{-}^{\prime \prime}\right)\right) .
\end{aligned}
$$

It is trivial to see that $\mathbf{C}_{0}$ is an isomorphism from $\left(\mathbb{R}^{6} \times \pi^{\prime \prime}\left(\mathcal{C}^{2, \alpha}\left(S^{1}\right)\right)\right)^{2}$ into $\left(\mathcal{E} \times \mathcal{C}^{1, \alpha}\left(S^{1}\right)\right)^{2}$. In particular, there is a unique zero of this mapping, namely where $\phi^{\prime \prime}=0$ and the $\left(w_{m}^{0}\right)_{ \pm}$are chosen to cancel $\pm \varepsilon \beta(r)$. Notice that this solution is certainly within $\mathcal{B}_{\kappa}^{2}$, because $|\varepsilon \beta(r)| \sim \varepsilon^{7 / 4}$.

We would like to use a degree theoretic argument to conclude that there is also a single zero of $\mathbf{C}_{\varepsilon}$ within $\mathcal{B}_{\kappa}^{2}$. Unfortunately, the nonlinear correction terms in the difference $\mathbf{C}_{\varepsilon}-\mathbf{C}_{0}$, whilst small, are not compact. We could, of course, use a contraction mapping argument again, but we propose, instead, the following shorter route. We write

$$
\begin{aligned}
\mathbf{C}_{\varepsilon}\left(\mathcal{A}, \phi^{\prime \prime}\right)= & \left(w_{m}^{0}+\varepsilon \beta(r)+F^{\prime}\left(\mathcal{A}, \phi^{\prime \prime}\right)+F^{\prime \prime}\left(\mathcal{A}, \phi^{\prime \prime}\right),\right. \\
& \left.\partial_{s}\left(w_{m}^{0}+\varepsilon \beta(r)\right)+\left(\eta_{\varepsilon}-1\right)\left|D_{\theta}\right| \phi^{\prime \prime}+\partial_{s} F^{\prime}\left(\mathcal{A}, \phi^{\prime \prime}\right)+\partial_{s} F^{\prime \prime}\left(\mathcal{A}, \phi^{\prime \prime}\right)\right) .
\end{aligned}
$$

Here $F^{\prime}=\left(I-\pi^{\prime \prime}\right)\left(\left(\mathbf{C}_{\varepsilon}-\mathbf{C}_{0}\right)\left(\mathcal{A}, \phi^{\prime \prime}\right)\right)$, and $F^{\prime \prime}=\pi^{\prime \prime}\left(\left(\mathbf{C}_{\varepsilon}-\mathbf{C}_{0}\right)\left(\mathcal{A}, \phi^{\prime \prime}\right)\right)$. The range of $F^{\prime}$ lies in the finite dimensional space $\mathcal{E}^{2}$, but the range of $F^{\prime \prime}$ is ostensibly the problem, since it is infinite dimensional. These error terms are all, however, $\mathcal{O}\left(\varepsilon^{3 / 2}\right)$, with constants independent of $\kappa$.

Define a family of smoothings of this map $\mathbf{C}_{\varepsilon, q}$, for $0<q<1$, by replacing the terms $F^{\prime \prime}\left(\mathcal{A}, \phi^{\prime \prime}\right)$ and $\partial_{s} F^{\prime \prime}\left(\mathcal{A}, \phi^{\prime \prime}\right)$ by $\left|D_{\theta}\right|^{-q} F^{\prime \prime}\left(\mathcal{A}, \phi^{\prime \prime}\right)$ and $\left|D_{\theta}\right|^{-q} \partial_{s} F^{\prime \prime}\left(\mathcal{A}, \phi^{\prime \prime}\right)$, respectively. Here $\left|D_{\theta}\right|^{-q}$ is the pseudodifferential operator of order $-q$ defined by

$$
\left|D_{\theta}\right|^{-q}: \sum_{|n| \geq 2} a_{n} e^{i n \theta} \longrightarrow \sum_{|n| \geq 2}|n|^{-q} a_{n} e^{i n \theta} .
$$


Since the norm of $\left|D_{\theta}\right|^{-q}$, when defined from $\mathcal{C}^{1, \alpha}\left(S^{1}\right)$ into itself, is bounded independently of $q$ for $0<q<1$, we see that the nonlinear terms are all still $\mathcal{O}\left(\varepsilon^{3 / 2}\right)$, independently of $\kappa$.

It is now commonplace, using the Leray-Schauder degree, that there exists $\left(\mathcal{A}, \phi^{\prime \prime}\right)_{q}$ for which $\mathbf{C}_{\varepsilon, q}\left(\left(\mathcal{A}, \phi^{\prime \prime}\right)_{q}\right)=0$. More specifically, this point exists in $\mathcal{B}_{\kappa}^{2}$ if we first choose $\kappa$ large enough to overwhelm the other ( $\kappa$-independent) constants which estimate the nonlinear terms in the mapping, and then choose $\varepsilon$ accordingly sufficiently small. We also note that $\left\|\left(\mathcal{A}, \phi^{\prime \prime}\right)_{q}\right\|$ is always in $\mathcal{B}_{\kappa}^{2}$, hence has norm bounded uniformly in $q$. This means that we may extract a sequence $q_{j} \rightarrow 0$ such that $\left(\mathcal{A}, \phi^{\prime \prime}\right)_{q_{j}}$ converges in $\mathcal{U} \times \mathcal{C}^{2, \alpha^{\prime}}$ for any fixed $\alpha^{\prime}<\alpha$. This is clearly sufficient for our purposes, and it is clear that the limit of this sequence is a zero of $\mathbf{C}_{\varepsilon}$. This completes our proof.

\section{Technical information needed for the proof of generic nondegeneracy}

Now that we have proven the existence of the family of CMC connected sums $S_{\varepsilon}$ of the two surfaces $\Sigma_{1}$ and $\Sigma_{2}$, we turn our attention to establishing criteria ensuring that the $S_{\varepsilon}$ are nondegenerate. This will require some preparatory work. In this section we give estimates on the graph function for $S_{\varepsilon}$ over the truncated rescaled catenoid and use this to describe the form of the Jacobi operator on $S_{\varepsilon}$. In the next section we give precise estimates for the solutions of this Jacobi operator corresponding to the low eigenmodes $j=0, \pm 1$ on the cross-section. After that we will be able to address the nondegeneracy question directly.

In the previous sections we gave good estimates for $S_{\varepsilon}$ as a graph over the truncated rescaled catenoid $\Sigma_{\varepsilon}^{c}$, specifically in the region where the parameter $s$ lies in $\left[-s_{\varepsilon}, s_{\varepsilon}\right]\left(s_{\varepsilon}=-(1 / 4) \log \varepsilon\right)$. However, we shall need to extend these estimates to the larger region including the balls $B_{\rho} \backslash B_{c \varepsilon^{3 / 4}}$ in each of the surfaces $\Sigma_{j}$. This entire region may be written as a graph over a region in $\Sigma_{\varepsilon}^{c}$. Recall the relationships between the various variables we have used:

$$
r=e^{-t} \quad \text { and } \quad e^{-t}=\varepsilon \cosh s .
$$

Since the annuli in $\Sigma_{j}$ are parametrized by $\left[\bar{t}, t_{\varepsilon}\right] \times S^{1}$, then if we define $\bar{s}_{\varepsilon}$ by

$$
e^{-\bar{t}}=\varepsilon \cosh \bar{s}_{\varepsilon},
$$

we see that the region in $S_{\varepsilon}$ of interest to us, which we write as $S_{\varepsilon} \cap B_{\rho}$, is parametrized by $\left[-\bar{s}_{\varepsilon}, \bar{s}_{\varepsilon}\right] \times S^{1}$.

Notice also that $S_{\varepsilon} \cap B_{\rho}$ may be decomposed into three components. The first central component, denoted by $I$, corresponds to $s$ lying in the interval $\left[-s_{\varepsilon}, s_{\varepsilon}\right]$. The two other components, $I I_{1}$ and $I I_{2}$, are vertical graphs over $\Sigma_{1}$ and $\Sigma_{2}$, respectively.

Lemma 2 For some small value of $\rho$, and for $\varepsilon$ sufficiently small, there is a function $g_{\varepsilon}$ on $\Sigma_{\varepsilon}^{c}$ such that

$$
\mathbf{x}_{\varepsilon}:\left[-\bar{s}_{\varepsilon}, \bar{s}_{\varepsilon}\right] \times S^{1} \ni(s, \theta) \longrightarrow \mathbf{x}_{\varepsilon}^{c}(s, \theta)+g_{\varepsilon}(s, \theta) \bar{n}_{\varepsilon}(s, \theta) \in S_{\varepsilon},
$$

where $\bar{n}_{\varepsilon}$ is the unit vector field on $\Sigma_{\varepsilon}^{c}$ defined in (24). Furthermore, the estimate

$$
\nabla^{k} g_{\varepsilon}(s, \theta)=\mathcal{O}\left(\varepsilon^{2} \cosh ^{2} s\right),
$$

holds for $(s, \theta) \in\left[-\bar{s}_{\varepsilon}, \bar{s}_{\varepsilon}\right] \times S^{1}$ when $k \geq 1$ but only for $(s, \theta) \in\left[-s_{\varepsilon}, s_{\varepsilon}\right] \times S^{1}$ when $k=0$.

Proof: In the region $I$, where $|s| \leq s_{\varepsilon}, g_{\varepsilon}=\tilde{w}+v$ as in Proposition 7, and so (77) follows directly from (33) and (34).

In the regions $I I_{i}$, when $s_{\varepsilon} \leq s \leq \bar{s}_{\varepsilon}, g_{\varepsilon}=\hat{w}_{m}+\tilde{w}+v$, and so we use the estimates in Proposition 14, (2) of Proposition 9 and (69). 
The restriction to $k \geq 1$ in the outer shell is simply because of the presence of the term $\varepsilon^{3 / 2}|\log \varepsilon|$ when $k=0$.

We also need the

Lemma 3 For $i=1,2$, the component $I I_{i}$ can be parametrized by

$$
\mathbf{x}_{i, \varepsilon}:(x, y) \in B_{\rho} \backslash B_{\varepsilon^{3 / 4}} \longrightarrow\left(x, y, u_{i}(x, y)+h_{i, \varepsilon}(x, y)\right)
$$

where $h_{i, \varepsilon}$ satisfies

$$
\nabla^{k} h_{i, \varepsilon}(x, y)=\mathcal{O}\left(r^{-k}\left(\varepsilon+\varepsilon^{3 / 4} r\right)\right)
$$

for $k \geq 1$.

The proof is similar to the proof of Lemma 2; the only difference is that $\hat{w}_{m}$ must be replaced by $w_{m}$. Details will be omitted. Again the restriction to $k \geq 1$ is simply to avoid a logarithmic term when $k=0$.

Finally, recall that $-\left(\varepsilon^{2} \cosh ^{2} s\right)^{-1} \mathcal{L}$ is the Jacobi operator about $\Sigma_{\varepsilon}^{c}$ with respect to the normal vector field $n$ while as in the expression following $(28),\left(\varepsilon^{2} \cosh ^{2} s\right)^{-1}\left(-\mathcal{L}+L_{\varepsilon}\right)$ is the Jacobi operator about this same surface with respect to the transverse vector field $\bar{n}_{\varepsilon}$. The coefficients of $L_{\varepsilon}$ are of order $1 /(\cosh s)^{2}$ and are supported in the region $s_{\varepsilon}-2 \leq|s| \leq s_{\varepsilon}-1$. We now let $\mathbb{L}_{\varepsilon}$ be the Jacobi operator on $S_{\varepsilon}$ with respect to $\bar{n}_{\varepsilon}$.

Corollary 5 When $(s, \theta) \in\left[-\bar{s}_{\varepsilon}, \bar{s}_{\varepsilon}\right] \times S^{1}$,

$$
\mathbb{L}_{\varepsilon}=-\frac{1}{\varepsilon^{2} \cosh ^{2} s}\left(\mathcal{L}-L_{\varepsilon}+\mathbb{L}_{\varepsilon}^{\prime}\right),
$$

where $\mathbb{L}_{\varepsilon}^{\prime}$ is a second order operator the coefficients of which, along with their derivatives, can be estimated by a constant times $\left(\varepsilon+\varepsilon^{2} \cosh ^{2} s\right)$ for $(s, \theta) \in\left[-\bar{s}_{\varepsilon}, \bar{s}_{\varepsilon}\right] \times S^{1}$.

Proof : Following (28), the mean curvature of any graph over the catenoid $\Sigma_{\varepsilon}^{c}$, parametrized using $\bar{n}_{\varepsilon}$, is given by

$$
\begin{array}{r}
-\frac{1}{\varepsilon^{2} \cosh ^{2} s} \quad\left(\mathcal{L} w-L_{\varepsilon} w+\varepsilon \bar{Q}_{\varepsilon}^{\prime}\left(\frac{w}{\varepsilon \cosh s}, \frac{\nabla w}{\varepsilon \cosh s}, \frac{\nabla^{2} w}{\varepsilon \cosh s}\right)\right. \\
\left.+\varepsilon \cosh s \bar{Q}_{\varepsilon}^{\prime \prime}\left(\frac{w}{\varepsilon \cosh s}, \frac{\nabla w}{\varepsilon \cosh s}, \frac{\nabla^{2} w}{\varepsilon \cosh s}\right)\right)
\end{array}
$$

The operator $\mathbb{L}_{\varepsilon}^{\prime}$ is obtained by linearizing the last two expressions around $g_{\varepsilon}$. Notice that when $|s| \geq s_{\varepsilon}, \bar{n}_{\varepsilon}$ is identically equal to $(0,0, \pm 1)$ and so by (42), the nonlinear terms only involve the derivatives of $g_{\varepsilon}$ and not $g_{\varepsilon}$ itself in this range, which means that we may use the estimate (77) in this region.

\section{Jacobi fields}

As we discussed at the beginning of the last section, we require precise asymptotics for the Jacobi fields for $\mathbb{L}_{\varepsilon}$ corresponding to the low eigenmodes on the circle. More specifically, there are explicit Jacobi fields on the catenoid, i.e. solutions of $\mathcal{L} w=0$ in $\mathbb{R} \times S^{1}$, given by

$$
\begin{array}{rlrl}
\Psi^{0,+}(s, \theta)=\tanh s, & \Psi^{0,-}(s, \theta) & =(1-s \tanh s), \\
\Psi^{ \pm 1,+}(s, \theta)=\frac{1}{\cosh s} e^{ \pm i \theta}, & \text { and } & \Psi^{ \pm 1,-}(s, \theta) & =\left(\frac{s}{\cosh s}+\sinh s\right) e^{ \pm i \theta} .
\end{array}
$$


These all arise from explicit families of perturbations of the catenoid. In fact, if $S$ is any CMC surface and $S(\eta)$ is a smooth one-parameter family of CMC deformations with $S(0)=S$, then $S(\eta)$ may be written as a graph (with respect to some transverse normal vector field) over $S$ for small $\eta$. Actually, all that is needed is that this graph function exist over any fixed compact set of $S$ for some nontrivial range of values of $\eta$ which might diminish to zero as the compact set grows. This is sufficient to make sense of the derivative of the graph function at $\eta=0$, and this derivative is a Jacobi field. The Jacobi fields above are obtained in this way, as derivatives of one parameter families of CMC surfaces parametrized using the unit normal vector field; $\Psi^{0,+}$ and $\Psi^{ \pm 1,+}$ correspond to vertical and horizontal translations, respectively, $\Psi^{0,-}$ corresponds to changes by dilation and $\Psi^{ \pm 1,-}$ correspond to rotations about the $x$ and $y$ axes. If we write the graphs using the vector field $\bar{n}_{\varepsilon}$ instead, then the corresponding Jacobi fields will be denoted $\bar{\Psi}_{\varepsilon}^{j, \pm}$. These are solutions of $\left(\mathcal{L}-L_{\varepsilon}\right) w=0$, and from (88) in Appendix I we have

$$
\bar{\Psi}_{\varepsilon}^{j, \pm}=\frac{1}{n \cdot \bar{n}_{\varepsilon}} \Psi^{j, \pm} .
$$

The goal of this section is to find good estimates for the Jacobi fields on the surfaces $S_{\varepsilon}$ which are perturbations of these; these will be solutions of $\mathbb{L}_{\varepsilon} w=0$ and will be denoted by $\Phi_{\varepsilon}^{j, \pm}$ for $j=0, \pm 1$. We are really only interested in describing them over the regions $I I_{i}, i=1,2$.

The five Jacobi fields which correspond to vertical and horizontal translations and rotations of the vertical axis are the easiest to describe. We shall only need to describe their behaviour over the regions $I I_{i}$, and will now use the variables $(x, y)$ rather than $(s, \theta)$ there.

Proposition 16 The Jacobi fields $\Phi_{\varepsilon}^{j,+}, j=0, \pm 1$, and $\Phi_{\varepsilon}^{ \pm 1,-}$ are described in $I I_{i}, i=1,2$, by

$$
\begin{gathered}
\Phi_{\varepsilon}^{0,+}(x, y)=(-1)^{i}, \\
\left.\left.\Phi_{\varepsilon}^{+1,+}(x, y)=(-1)^{i} \partial_{x} \tilde{u}_{i, \varepsilon}(x, y)\right), \quad \Phi_{\varepsilon}^{-1,+}(x, y)=(-1)^{i} \partial_{y} \tilde{u}_{i, \varepsilon}(x, y)\right)
\end{gathered}
$$

and

$\Phi_{\varepsilon}^{+1,-}(x, y)=(-1)^{i}\left(x+\tilde{u}_{i, \varepsilon}(x, y) \partial_{x} \tilde{u}_{i, \varepsilon}(x, y)\right), \quad \Phi_{\varepsilon}^{-1,-}(x, y)=(-1)^{i}\left(y+\tilde{u}_{i, \varepsilon}(x, y) \partial_{y} \tilde{u}_{i, \varepsilon}(x, y)\right)$.

where $\tilde{u}_{i, \varepsilon} \equiv u_{i}+h_{i, \varepsilon}$.

Proof: The simple expression for $\Phi_{\varepsilon}^{0,+}$ follows from the fact that $\bar{n}_{\varepsilon}=\left(0,0,(-1)^{i+1}\right)$ in $I I_{i}$. On the other hand, recall from Lemma 3 that in these regions the graph functions for $S_{\varepsilon}$ relative to $\bar{n}_{\varepsilon}$ have the form $u_{i}(x, y)+h_{i, \varepsilon}(x, y)$. Differentiating with respect to $x$ and $y$ corresponds to infinitesimal translations in these directions, and this leads to the stated expressions. The Jacobi fields corresponding to the two rotations of the vertical axis can be obtained similarly.

Unfortunately, it is more difficult to get good estimates for the last remaining Jacobi field since we have not proved that $S_{\varepsilon}$ depends smoothly on $\varepsilon$. We will obtain this last function, and estimates for it, by a perturbation argument.

Proposition 17 Assume that $\delta \in(1,2)$. Then for some $\bar{s}_{1}>0$ sufficiently large, but independent of $\varepsilon$, and $\varepsilon$ is small enough, there exists a Jacobi field $\Phi_{\varepsilon}^{0,-}$, defined in $\left[-\bar{s}_{\varepsilon}+\bar{s}_{1}, \bar{s}_{\varepsilon}-\bar{s}_{1}\right] \times S^{1}$, which satisfy

$$
\Phi_{\varepsilon}^{0,-}(x, y)=-\log (2 r / \varepsilon)+\mathcal{O}\left(r+r^{\delta}|\log \varepsilon|\right),
$$

in $\mathbf{x}_{i, \varepsilon}\left(B_{\bar{\rho}_{1}} \backslash B_{\varepsilon^{3 / 4}}\right)$, for $i=1,2$. By definition here, $\bar{\rho}_{1} \equiv \varepsilon \cosh \left(\bar{s}_{\varepsilon}-\bar{s}_{1}\right)$.

Proof: First, by (88) in Appendix I,

$$
\mathbb{L}_{\varepsilon}\left(\frac{1}{n \cdot \bar{n}_{\varepsilon}} w\right)=-\frac{1}{\varepsilon^{2} \cosh ^{2} s} \mathcal{L} w+\frac{1}{\varepsilon^{2} \cosh ^{2} s} \mathbb{L}_{\varepsilon}^{\prime \prime} w
$$


where the operator $\mathbb{L}_{\varepsilon}^{\prime \prime}$ enjoys the same properties as $\mathbb{L}_{\varepsilon}^{\prime}$, namely has all its coefficients bounded by a constant times $\varepsilon+\varepsilon^{2} \cosh ^{2} s$. Therefore, it is enough to find the appropriate Jacobi fields for the operator

$$
\mathcal{L}-\mathbb{L}_{\varepsilon}^{\prime \prime}
$$

First, if $\bar{s}_{1}$ is chosen large enough, the result of Proposition 1 holds for all $\varepsilon$ small enough, with $s_{0}=\bar{s}_{\varepsilon}-\bar{s}_{1}$ and with $\mathcal{L}$ replaced by $\mathcal{L}-\mathbb{L}_{\varepsilon}^{\prime \prime}$. Indeed,

$$
\left\|\mathbb{L}_{\varepsilon}^{\prime \prime}(w)\right\|_{0, \alpha, \delta} \leq c\left(\varepsilon+e^{-2 \bar{s}_{1}}\right)\|w\|_{2, \alpha, \delta} .
$$

The claim follows immediately, provided $\bar{s}_{1}$ is chosen large enough. From now on we keep $\bar{s}_{1}$ fixed so that this is true and we will denote by $\mathbb{G}_{\bar{s}_{\varepsilon}-\overline{s_{1}}}$ the right inverse obtained by perturbing the right inverse $\mathcal{G}_{\bar{s}_{\varepsilon}-\bar{s}_{1}}$ for $\mathcal{L}$.

We obtain the desired function

$$
\Phi_{\varepsilon}^{0,-}=\Psi^{0,-}-\mathbb{G}_{\bar{s}_{\varepsilon}-\bar{s}_{1}}\left(\mathbb{L}_{\varepsilon}^{\prime \prime}\left(\Psi^{0,-}\right)\right)
$$

easily enough.

The main work will be in estimating $\mathcal{G}_{\bar{s}_{\varepsilon}-\bar{s}_{1}}\left(\mathbb{L}_{\varepsilon}^{\prime \prime}\left(\Psi^{0,-}\right)\right)$. First, recall that $r=\varepsilon \cosh s$. This implies that when $s>0$,

$$
\varepsilon e^{s}=2 r+\mathcal{O}\left(\varepsilon^{2} r^{-1}\right), \quad \varepsilon e^{-s}=\mathcal{O}\left(\varepsilon^{2} r^{-1}\right), \quad \text { and } \quad s=\log (2 r / \varepsilon)+\mathcal{O}\left(\varepsilon^{2} / r^{2}\right),
$$

and so

$$
1-s \tanh s=1-\log (2 r / \varepsilon)+\mathcal{O}\left(\varepsilon^{2}|\log \varepsilon| r^{-2}\right) .
$$

On the other hand, when $s<0$,

$$
\varepsilon e^{-s}=2 r+\mathcal{O}\left(\varepsilon^{2} r^{-1}\right), \quad \varepsilon e^{s}=\mathcal{O}\left(\varepsilon^{2} r^{-1}\right), \quad \text { and } \quad s=-\log (2 r / \varepsilon)+\mathcal{O}\left(\varepsilon^{2} / r^{2}\right),
$$

which gives

$$
1-s \tanh s=1-\log (2 r / \varepsilon)+\mathcal{O}\left(\varepsilon^{2}|\log \varepsilon| r^{-2}\right) .
$$

We next show that we can get somewhat sharper estimates for $\mathcal{G}_{\bar{s}_{\varepsilon}-\bar{s}_{1}}\left(\mathbb{L}_{\varepsilon}^{\prime \prime}\left(\Psi^{j, \pm}\right)\right)$ than those obtained from Proposition 4 directly. Using the bounds on the coefficients of $\mathbb{L}_{\varepsilon}^{\prime \prime}$ we find that

$$
\mid\left(\mathbb{L}_{\varepsilon}^{\prime \prime}\left(\Psi^{0,-}\right)\left|\leq c\left(\varepsilon+\varepsilon^{2} \cosh ^{2} s\right)(1+|s|) \leq c\left(\varepsilon+\varepsilon^{\delta} \cosh ^{\delta} s\right)\right| \log \varepsilon \mid,\right.
$$

for some constants $c>0$ which are independent of $\varepsilon$. We have also estimated $(\varepsilon \cosh s)^{k}, k=2,3$, by $\varepsilon^{\delta} \cosh ^{\delta} s$ here in order to simplify later estimates.

Now recall the construction of Proposition 4 . Let us write

$$
w=\mathcal{G}_{\bar{s}_{\varepsilon}-\bar{s}_{1}}\left(\mathbb{L}_{\varepsilon}^{\prime \prime}\left(\Psi^{0,-}\right)\right)=\sum_{n \in \mathbb{Z}} w_{n}(s) e^{i n \theta}, \quad f=\mathbb{L}_{\varepsilon}^{\prime \prime}\left(\Psi^{0,-}\right)=\sum_{n \in \mathbb{Z}} f_{n}(s) e^{i n \theta} .
$$

As in that proof, when $|n| \geq 2$, multiples of the function $n^{-2}\left(\varepsilon+\varepsilon^{\delta}(\cosh s)^{\delta}\right)|\log \varepsilon|$ can be used as supersolutions for $\pm w_{n}$. Hence, for $|n| \geq 2$,

$$
\left|w_{n}(s)\right| \leq \frac{c}{n^{2}}\left(\varepsilon+\varepsilon^{\delta} \cosh ^{\delta} s\right)|\log \varepsilon| .
$$

To handle the remaining cases $n=0, \pm 1$ we use the explicit formulæ (16) and (17)

$$
w_{0}(s)=\tanh s \int_{0}^{s} \tanh ^{-2} t \int_{0}^{t} \tanh u f_{0}(u) d u d t,
$$

and

$$
w_{ \pm 1}(s)=\cosh ^{-1} s \int_{0}^{s} \cosh ^{2} t \int_{0}^{t} \cosh ^{-1} u f_{ \pm 1}(u) d u d t
$$


Direct estimates yield

$$
\left|w_{j}(s)\right| \leq c\left(\varepsilon|\log \varepsilon|^{3}+\varepsilon \cosh s+\varepsilon^{\delta} \cosh ^{\delta} s|\log \varepsilon|\right) \leq c\left(\varepsilon \cosh s+r^{\delta}|\log \varepsilon|\right), \quad j=0, \pm 1 .
$$

Summation over $n$ now yields the desired estimate for the remainder term. The derivatives are handled similarly.

\section{Proof of generic nondegeneracy}

Fix $\left(p_{1}, p_{2}, \theta\right) \in \Sigma_{1} \times \Sigma_{2} \times S^{1}$, and then choose rigid motions of the surfaces $\Sigma_{j}$ so that the points $p_{j}$ are mapped to the origin and the tangent planes $T_{p_{j}} \Sigma_{j}$ are mapped to the $x y$-plane with opposite orientation. Suppose furthermore that we first normalize these mappings so that the principal directions at these points are mapped to the $x$ and $y$ axes, respectively. (There is of course a choice to be made here regarding the ordering of the principal directions, but we require, for example, that the direction with larger principal curvature be carried to the $x$-axis; since we are specifying an orientation, this fixes the choice at all points except umbilics.) Finally, rotate $\Sigma_{2}$ about the $z$-axis by an angle $\theta$ so that its principal directions are aligned with the vectors $(\cos \theta, \sin \theta, 0)$ and $(-\sin \theta, \cos \theta, 0)$. We call the resulting singular configuration $\Sigma_{1} \sqcup \Sigma_{2}\left(p_{1}, p_{2}, \theta\right)$. The resulting 'moduli space', $C\left(\Sigma_{1}, \Sigma_{2}\right)$, of such configurations is clearly five dimensional. It is the quotient of an eleven-dimensional space by the (six-dimensional) group of rigid motions. This procedure yields local charts on $C\left(\Sigma_{1}, \Sigma_{2}\right)$. Finally, given some sufficiently small $\varepsilon>0$, we form the desingularized connected sum $S_{\varepsilon}\left(p_{1}, p_{2}, \theta\right)$. Note that $\Sigma_{1} \sqcup \Sigma_{2}\left(p_{1}, p_{2}, \theta\right)$ is the union, near the origin, of two graphs over the $x y$-plane

$$
\mathbf{x}_{i}: B_{\rho} \ni(x, y) \longrightarrow\left(x, y, u_{i}(x, y)\right), \quad i=1,2 .
$$

The maps $\mathbf{x}_{i}$ and $u_{i}$ depend, of course, on $p_{1}, p_{2}$ and $\theta$.

Our aim in this final section is to prove Proposition 3, that is, to prove the nondegeneracy of $S_{\varepsilon}\left(p_{1}, p_{2}, \theta\right)$ for $\varepsilon$ small. Recall that this means that we need to show that there are no nontrivial Jacobi fields on $S_{\varepsilon}\left(p_{1}, p_{2}, \theta\right)$ which vanish on $\partial S_{\varepsilon}\left(p_{1}, p_{2}, \theta\right)$. We are not able to show that this is true for every value of the parameters, but at least we shall show that it holds generically, in a precise sense.

We first prove a result which gives a criterion for nondegeneracy.

Theorem 2 Let $\left(p_{1}, p_{2}, \theta\right) \in \Sigma_{1} \times \Sigma_{2} \times S^{1}$ be fixed. If there exists a sequence $\varepsilon_{n} \rightarrow 0$ for which the surface $S_{\varepsilon_{n}}\left(p_{1}, p_{2}, \theta\right)$ is degenerate, then

$$
\left(\partial_{x x}^{2} u_{2}(0,0)-\partial_{x x}^{2} u_{1}(0,0)\right)\left(\partial_{y y}^{2} u_{2}(0,0)-\partial_{y y}^{2} u_{1}(0,0)\right)-\left(\partial_{x y}^{2} u_{2}(0,0)-\partial_{x y}^{2} u_{1}(0,0)\right)^{2}=0 .
$$

Proof: We omit $p_{1}, p_{2}$ and $\theta$ from the notation since they are fixed. Let $\mathbb{L}_{\varepsilon}$ denote the mean curvature operator linearized about $S_{\varepsilon}$ with respect to the normal transversal vector field used in the previous section. We shall also often simply write $\varepsilon$ instead of $\varepsilon_{n}$. The degeneracy of $S_{\varepsilon}$ means that there exists a nontrivial function $w_{\varepsilon}$ on $S_{\varepsilon}$ with $w_{\varepsilon}=0$ on $\partial S_{\varepsilon}$ and such that $\mathbb{L}_{\varepsilon} w_{\varepsilon}=0$.

Fix any $\delta_{0} \in(1,2)$. We now choose, for each $\varepsilon$, a weight function $\gamma_{\varepsilon}: S_{\varepsilon} \rightarrow \mathbb{R}$ which satisfies

$$
\gamma_{\varepsilon}(p) \equiv 1 \quad \text { in } \quad S_{\varepsilon} \backslash B_{2 \rho}(0), \quad \gamma_{\varepsilon} \circ \mathbf{x}_{i, \varepsilon}(t, \theta) \equiv e^{\delta_{0} t} \quad \text { in } \quad\left[\bar{t}_{1}, t_{\varepsilon}\right] \times S^{1},
$$

for $i=1,2$, and

$$
\gamma_{\varepsilon} \circ \mathbf{x}_{\varepsilon}(s, \theta) \equiv(\varepsilon \cosh s)^{-\delta_{0}} \quad \text { in } \quad\left[-s_{\varepsilon}, s_{\varepsilon}\right] \times S^{1} .
$$

We also require that $\gamma_{\varepsilon}$ and its derivative are bounded independently of $\varepsilon$ in $S_{\varepsilon} \cap\left(B_{2 \rho} \backslash B_{\rho}\right)$. 
Use these weight functions to normalize the functions $w_{\varepsilon}$ by

$$
\sup _{p \in S_{\varepsilon}} \gamma_{\varepsilon}(p)\left|w_{\varepsilon}(p)\right|=1
$$

Suppose that $p_{\varepsilon} \in S_{\varepsilon}$ is a point where this supremum is achieved. Passing to a subsequence, we may assume that $\left\{p_{\varepsilon}\right\}$ converges to some point $p_{\infty} \in \Sigma_{1} \cup \Sigma_{2}$. We distinguish various cases according to the location of $p_{\infty}$.

Case 1. Assume that $p_{\infty}=0$. In this case, we may write (at least for $\varepsilon$ small enough)

$$
p_{\varepsilon}=\mathbf{x}_{\varepsilon}\left(s_{\varepsilon}^{\prime}, \theta_{\varepsilon}^{\prime}\right)
$$

for some $\left(s_{\varepsilon}^{\prime}, \theta_{\varepsilon}^{\prime}\right) \in[\log \varepsilon+c,-\log \varepsilon-c] \times S^{1}$. We distinguish two further cases according to the behaviour of the sequence $s_{\varepsilon}^{\prime}$.

Subcase 1.1. Assume that (up to a subsequence) $\left(s_{\varepsilon}^{\prime}, \theta_{\varepsilon}^{\prime}\right)$ converges to $\left(s_{0}, \theta_{0}\right) \in \mathbb{R} \times S^{1}$. Then define

$$
\tilde{w}_{\varepsilon}(s, \theta)=\varepsilon^{\delta_{0}} w_{\varepsilon} \circ \mathbf{x}_{\varepsilon}(s, \theta) .
$$

This still solves $\mathbb{L}_{\varepsilon} \tilde{w}_{\varepsilon}=0$ in $[\log \varepsilon-c,-\log \varepsilon+c] \times S^{1}$, is bounded by $(\cosh s)^{-\delta_{0}}$ and also satisfies

$$
\tilde{w}_{\varepsilon}\left(s_{\varepsilon}^{\prime}, \theta_{\varepsilon}^{\prime}\right) \equiv 1
$$

Now pass to the limit, possibly after passing to a further subsequence. By Corollary 0 we obtain a nontrivial function $w$ such that

$$
\partial_{s s}^{2} w+\partial_{\theta \theta}^{2} w+\frac{2}{\cosh ^{2} s} w=0
$$

in $\mathbb{R} \times S^{1}$ and which is bounded by $(\cosh s)^{-\delta_{0}}$. We now show that this is not possible. Let

$$
w(s, \theta)=\sum_{n \in \mathbb{Z}} w_{n}(s) e^{i n \theta}
$$

be the Fourier decomposition of $w$. Then

$$
\hat{w}(s, \theta)=\sum_{|n| \geq 2} w_{n}(s) e^{i n \theta},
$$

is still a solution of (82); it also decays exponentially at both $\pm \infty$. Multiplying (82) by $\hat{w}$ and integrating by parts we find

$$
\int_{\mathbb{R} \times S^{1}}\left(\left(\partial_{s} \hat{w}\right)^{2}+\left(\partial_{\theta} \hat{w}\right)^{2}-\frac{2}{\cosh ^{2} s} \hat{w}^{2}\right) d s d \theta=0,
$$

which implies that $\hat{w}=0$. Hence $w=\sum_{|n| \leq 1} w_{n}(s) e^{i n \theta}$. As in the last section, the solutions in these low eigenspaces are linear combinations of the explicit solutions $\Psi^{j, \pm}, j=0, \pm 1$, and no nontrivial solution of this form can decay as quickly as $(\cosh s)^{-\delta_{0}}$ at $\pm \infty$. Hence this subcase cannot occur.

Subcase 1.2. Now assume that $\lim _{\varepsilon \rightarrow 0} s_{\varepsilon}^{\prime}=+\infty$ or $-\infty$. To fix ideas, assume that $\lim _{\varepsilon \rightarrow 0} s_{\varepsilon}^{\prime}=$ $-\infty$. Notice that because $\lim _{\varepsilon \rightarrow 0} p_{\varepsilon}=0$, we also have $\lim _{\varepsilon \rightarrow 0} s_{\varepsilon}^{\prime}-\log \varepsilon=+\infty$. Define

$$
\hat{w}_{\varepsilon}(s, \theta)=\varepsilon^{\delta_{0}}\left(\cosh s_{\varepsilon}^{\prime}\right)^{\delta_{0}} w_{\varepsilon}\left(s+s_{\varepsilon}^{\prime}, \theta\right) .
$$

This function is bounded by a constant times $e^{\delta_{0} s}$ in $\left[\log \varepsilon-s_{\varepsilon}^{\prime},-s_{\varepsilon}^{\prime}\right] \times S^{1}$ and satisfies

$$
\lim _{\varepsilon \rightarrow 0} \hat{w}_{\varepsilon}\left(0, \hat{\theta}_{\varepsilon}\right)=1 \text {. }
$$


Again passing to the limit as $\varepsilon \rightarrow 0$ using Corollary , we obtain a nontrivial solution of

$$
\partial_{s s}^{2} w+\partial_{\theta \theta}^{2} w=0 \quad \text { in } \quad \mathbb{R} \times S^{1},
$$

which is bounded by $e^{-\delta_{0} s}$. Since $\delta_{0} \notin \mathbb{Z}$, this is impossible, which rules out this subcase.

Case 2. Finally we assume that $\lim _{\varepsilon \rightarrow 0} p_{\varepsilon} \neq 0$. Possibly extracting subsequences, we pass to the limit as $\varepsilon$ tends to 0 and obtain two solutions $w_{1}$ and $w_{2}$ (at least one of which is nontrivial) of

$$
\Lambda_{i} w_{i}=0 \quad \text { in } \quad \Sigma_{i} \backslash\{0\}
$$

with $w_{i}=0$ on $\partial \Sigma_{i}$. We know that $w_{i} \in \mathcal{C}_{-\delta_{0}}^{2, \alpha}\left(\Sigma_{i} \backslash\{0\}\right)$, and so there must exist constants $c_{j}^{i} \in \mathbb{R}$, $j=0, \pm 1$, such that

$$
\Lambda_{i} w_{i}=-2 \pi\left(c_{0}^{i} \delta_{0}+\left(c_{1}^{i}, c_{2}^{i}\right) \cdot \nabla \delta_{0}\right) .
$$

Our goal is to show that these constants $c_{j}^{i}$ all vanish. We claim that this follows from the condition (81). Granting this, then each $w_{i}$ must be a regular Jacobi fields over the whole of $\Sigma_{i}$, and at least one of them must be nontrivial. Nondegeneracy of the two surfaces implies that both $w_{1}=0$ and $w_{2}=0$, which is a contradiction.

Therefore, it remains to prove this claim. Choose some $s_{1}>\bar{s}_{1}$ to be fixed later. We now use the variables $(x, y)$ and set

$$
r_{1} \equiv \varepsilon \cosh \left(\bar{s}_{\varepsilon}-s_{1}\right)
$$

Then the boundary of $\left[-\bar{s}_{\varepsilon}+s_{1}, \bar{s}_{\varepsilon}-s_{1}\right] \times S^{1}$ consists of two circles of radius $r_{1}$, one in each of the regions $I I_{1}$ and $I I_{2}$, which we denote by $\partial B_{r_{1}}^{i}$. Also, set for $i=1,2$

$$
\tilde{u}_{i, \varepsilon} \equiv u_{i}+h_{\varepsilon, i} .
$$

Now multiply $\mathbb{L}_{\varepsilon} w_{\varepsilon}=0$ by any one of the 'low eigenmode' Jacobi fields $\Phi=\Phi_{\varepsilon}^{j, \pm}, j=0, \pm 1$, and integrate over $\left[-\bar{s}_{\varepsilon}+s_{1}, \bar{s}_{\varepsilon}-s_{1}\right] \times S^{1}$. If we set

$$
J^{i} \equiv\left(\int_{\partial B_{r_{1}}^{i}} \frac{\Phi \partial_{r} w-w \partial_{r} \Phi}{\left(1+\left|\nabla \tilde{u}_{i, \varepsilon}\right|^{2}\right)^{1 / 2}} r d \theta-\int_{\partial B_{r_{1}}^{i}} \frac{\nabla \tilde{u}_{i, \varepsilon} \cdot(\Phi \nabla w-w \nabla \Phi)}{\left(1+\left|\nabla \tilde{u}_{i, \varepsilon}\right|^{2}\right)^{3 / 2}} \partial_{r} \tilde{u}_{i, \varepsilon} r d \theta\right),
$$

then we obtain by integration by parts and (42) that

$$
J^{1}+J^{2}=0 .
$$

We substitute in each of the Jacobi fields in turn into this equality to get different information. First of all, we note that the estimates for $h_{\varepsilon, i}$ in Lemma 3 show that $\left|\nabla \tilde{u}_{i, \varepsilon}\right| \leq c|\nabla u| \leq c^{\prime} r$. It may then be checked that the first integral in $J^{i}$ always contains the dominant terms of the expansion with respect to $r$, and furthermore, that the denominator $\left(1+\left|\nabla \tilde{u}_{i, \varepsilon}\right|^{2}\right)^{1 / 2}$ in this integral may be replaced by 1 without affecting the first two terms of the expansion. Hence we shall really be only computing the leading asymptotic terms in (84) as $\varepsilon \rightarrow 0$. Finally, we note that

$$
w_{i}=(-1)^{i+1}\left(\frac{c_{1}^{i} \cos \theta+c_{2}^{i} \sin \theta}{r}+c_{0}^{i} \log r+\mathcal{O}(1)\right) .
$$

First we set $\Phi=\Phi_{\varepsilon}^{0,+}$. Since $\Phi_{\varepsilon}^{0,+}=(-1)^{i}$ in $I I^{i}$, we get that

$$
J^{i} \sim-\int\left(\frac{-\left(c_{1}^{i} \cos \theta+c_{2}^{i} \sin \theta\right)}{r^{2}}+\frac{c_{0}^{i}}{r}+\mathcal{O}(1)\right) r d \theta+\mathcal{O}\left(r_{1}\right) .
$$

The coefficient of $r^{-2}$ integrates to zero, and so

$$
c_{0}^{1}+c_{0}^{2}=\mathcal{O}\left(e^{-s_{1}}\right) .
$$


Since, this holds for every $s_{1}$, we conclude $c_{0}^{1}+c_{0}^{2}=0$.

Next let $\Phi=\Phi^{ \pm 1,-}$. Using Proposition 17, the leading terms of the expansion is

$$
J^{i} \sim 2 \int\left(c_{1}^{1} \cos \theta+c_{2}^{1} \sin \theta\right) S d \theta+\mathcal{O}\left(r_{1}\right),
$$

where $S$ is equal to either $\cos \theta$ or $\sin \theta$. This gives

$$
c_{1}^{1}+c_{1}^{2}=c_{2}^{1}+c_{2}^{2}=0 .
$$

When $\Phi=\Phi^{ \pm 1,+}$ then we use Proposition 16 along with the fact that $u_{i}$ may be approximate by its second order Taylor polynomial and (as before) $h_{i, \varepsilon}$ may be disregarded. This gives

$$
\begin{array}{r}
c_{1}^{1} \partial_{x x}^{2} u_{1}+c_{2}^{1} \partial_{x y}^{2} u_{1}+c_{1}^{2} \partial_{x x}^{2} u_{2}+c_{2}^{2} \partial_{x y}^{2} u_{2}=0, \quad \text { and } \\
c_{1}^{1} \partial_{x y}^{2} u_{1}+c_{2}^{1} \partial_{y y}^{2} u_{1}+c_{1}^{2} \partial_{x y}^{2} u_{2}+c_{2}^{2} \partial_{y y}^{2} u_{2}=0
\end{array}
$$

with all partial derivatives computed at the origin. We write these equations all together as

$$
\left(\begin{array}{cccc}
1 & 0 & 1 & 0 \\
0 & 1 & 0 & 1 \\
\partial_{x x}^{2} u_{1} & \partial_{x y}^{2} u_{1} & \partial_{x x}^{2} u_{2} & \partial_{x y}^{2} u_{2} \\
\partial_{x y}^{2} u_{1} & \partial_{y y}^{2} u_{1} & \partial_{x y}^{2} u_{2} & \partial_{y y}^{2} u_{2}
\end{array}\right)\left(\begin{array}{c}
c_{1}^{1} \\
c_{2}^{1} \\
c_{1}^{2} \\
c_{2}^{2}
\end{array}\right)=\left(\begin{array}{l}
0 \\
0 \\
0 \\
0
\end{array}\right)
$$

Since we are assuming that (81) does not hold, this matrix is not singular and so $c_{1}^{1}=c_{2}^{1}=c_{1}^{2}=$ $c_{2}^{2}=0$.

Finally, we let $\Phi=\Phi_{\varepsilon}^{0,-}$. We have already shown that $c_{j}^{i}=0$ when $j=1,2$, and the leading term of $\Phi$ is $\log \varepsilon$. Then the leading singular term in the expansion for $J^{i}$ is

$$
\frac{J^{i}}{\log \varepsilon} \sim(-1)^{i+1} \int c_{0}^{i} d \theta+\mathcal{O}\left(r_{1}\right)
$$

Hence we get $c_{0}^{1}=c_{0}^{2}$, which together with the fact that $c_{0}^{1}+c_{0}^{2}=0$, implies that $c_{0}^{1}=c_{0}^{2}=0$. The claim, and the theorem, is now proved.

Using this result, the proof of Theorem 2 is now easy to complete. In fact, we merely translate (81) into a more explicit equation involving the principle curvatures of the surfaces $\Sigma_{i}$ at the points $p_{i}$ and the angle $\theta$. We shall denote the principle curvatures of $\Sigma_{i}$ by $\alpha_{i}$ and $\beta_{i}$.

Recall that we had oriented the surfaces so that the $x$ and $y$ axes are principle directions for $\Sigma_{1}$. Thus

$$
\partial_{x x}^{2} u_{1}(0,0)=\alpha_{1}, \quad \partial_{x y}^{2} u_{1}(0,0)=0, \quad \text { and } \quad \partial_{y y}^{2} u_{1}(0,0)=\beta_{1} .
$$

On the other hand, using coordinates $(\tilde{x}, \tilde{y})$ defined by $\tilde{x}=\cos \theta x+\sin \theta y$ and $\tilde{y}=-\sin \theta x+$ $\cos \theta y$, we conclude that

$$
\begin{gathered}
\partial_{x x}^{2} u_{2}(0,0)=-\alpha_{2} \cos ^{2} \theta-\beta_{2} \sin ^{2} \theta, \quad \partial_{x y}^{2} u_{2}(0,0)=\left(\beta_{2}-\alpha_{2}\right) \sin \theta \cos \theta, \\
\text { and } \partial_{y y}^{2} u_{2}(0,0)=-\alpha_{2} \sin ^{2} \theta-\beta_{2} \cos ^{2} \theta
\end{gathered}
$$

(Recall that $\Sigma_{2}$ is oppositely oriented to $\Sigma_{1}$, which accounts for the change of signs.)

We have now proved that the surface $S_{\varepsilon}$ can be degenerate for $\varepsilon$ sufficiently small only if

$$
\left(\alpha_{2} \cos ^{2} \theta+\beta_{2} \sin ^{2} \theta+\alpha_{1}\right)\left(\alpha_{2} \sin ^{2} \theta+\beta_{2} \cos ^{2} \theta+\beta_{1}\right)-\sin ^{2} \theta \cos ^{2} \theta\left(\beta_{2}-\alpha_{2}\right)^{2}=0 .
$$


Some algebra shows that this is equivalent to

$$
\left(\alpha_{1} \alpha_{2}+\beta_{1} \beta_{2}\right) \sin ^{2} \theta+\left(\alpha_{1} \beta_{2}+\beta_{1} \alpha_{2}\right) \cos ^{2} \theta+\left(\alpha_{1} \beta_{1}+\alpha_{2} \beta_{2}\right)=0 .
$$

This is equivalent to a quadratic polynomial in $\cos \theta$, and hence either the polynomial is identically satisfied, or else there are at most two values of $\cos \theta$ for which it vanishes (and hence at most four values of $\theta$ ). The polynomial can only be identically satisfied if

$$
\alpha_{1} \alpha_{2}+\beta_{1} \beta_{2}=\alpha_{1} \beta_{2}+\beta_{1} \alpha_{2}=-\left(\alpha_{1} \beta_{1}+\alpha_{2} \beta_{2}\right) .
$$

Recalling that $\beta_{i}=H_{0}-\alpha_{i}$, the first equality implies that $\alpha_{1} H_{0}+\alpha_{2} H_{0}=2 \alpha_{1} \alpha_{2}+H_{0}^{2} / 2$ while the second gives $\left(\alpha_{1}+\alpha_{2}\right)\left(2 H_{0}-\alpha_{1}-\alpha_{2}\right)=0$. These equations together yield $\alpha_{1}=H_{0} / 2$, $\alpha_{2}=3 H_{0} / 2$, and hence $\beta_{1}=H_{0} / 2, \beta_{2}=-H_{0} / 2$, so that $\Sigma_{1}$ is umbilic at $p_{1}$, or else $\Sigma_{2}$ is umbilic at $p_{2}$ (with principal curvatures $\left(H_{0} / 2, H_{0} / 2\right)$ ) while the principal curvatures of $\Sigma_{1}$ at $p_{1}$ are $3 H_{0} / 2$ and $-H_{0} / 2$.

To proceed further, we show that the set of points in $\Sigma_{1} \times \Sigma_{2}$ where the principal curvatures can have these set values is no more than three dimensional. The real analyticity of CMC surfaces shows that the locus of points with fixed principal curvatures is an analytic set, hence either a discrete set, a collection of analytic arcs or else the whole surface. Now by definition an isoparametric surface $\Sigma$ is one for which the principal curvatures are everywhere constant. It is a classical theorem of Cartan that the only isoparametric surfaces in $\mathbb{R}^{3}$, even locally, are subdomains of the sphere and the cylinder. Hence although $\Sigma_{1}$ could be everywhere umbilic, it is impossible for $\Sigma_{2}$ to have principal curvatures $\left(3 H_{0} / 2,-H_{0} / 2\right)$ on an open set. This shows that the portion of the degeneracy set $\mathcal{S}$ which includes the complete $S^{1}$ factor lies over a set in $\Sigma_{1} \times \Sigma_{2}$ which is three dimensional if one of the $\Sigma_{i}$ is a subdomain of the sphere, and at most two dimensional otherwise.

The proof of Proposition 3 is now complete.

\section{Appendix I : Using different vector fields to parameter- ize all nearby surfaces}

This section is entirely taken from [9]. We have included it here for the sake of completeness. Let $\Sigma$ be a regular orientable surface, with unit normal vector field $N$. Suppose that $\bar{N}$ is another unit vector field along $\Sigma$ which is nowhere tangential. By the inverse function theorem, for any $p_{0} \in S$ there are neighbourhoods $\mathcal{U}$ and $\mathcal{V}$ near $\left(p_{0}, 0\right)$ in $\Sigma \times \mathbb{R}$ and a diffeomorphism $(\phi(p, s), \psi(p, s))$ from $\mathcal{U}$ to $\mathcal{V}$ such that

$$
p+s N(p)=\phi(p, s)+\psi(p, s) \bar{N}(\phi(p, s)) .
$$

Here $\phi(p, 0)=p$ and $\psi(p, 0)=0$. To determine the first order Taylor series of these functions in $s$, differentiate (85) with respect to $s$ and set $s=0$. This gives

$$
N(p)=\frac{\partial \phi}{\partial s}(p, 0)+\frac{\partial \psi}{\partial s}(p, 0) \bar{N}(p),
$$

and so, taking the normal component of this, we get

$$
1=\frac{\partial \psi}{\partial s}(p, 0) N(p) \cdot \bar{N}(p), \quad \text { or } \quad \frac{\partial \psi}{\partial s}(p, 0)=1 /(N(p) \cdot \bar{N}(p)) .
$$

Hence

$$
\psi(p, s)=\frac{s}{N(p) \cdot \bar{N}(p)}+O\left(s^{2}\right) .
$$

On the other hand, taking the tangential component and using this expansion of $\psi$ yields

$$
0=\frac{\partial \phi}{\partial s}(p, 0)+\frac{s}{N(p) \cdot \bar{N}(p)} \bar{N}_{t}(p)
$$


where $\bar{N}_{t}(p)$ is the tangential component of $\bar{N}$. Thus

$$
\phi(p, s)=p-\frac{s}{N(p) \cdot \bar{N}(p)} \bar{N}_{t}(p)+O\left(s^{2}\right) .
$$

Next, any $\mathcal{C}^{2}$ surface close to $\Sigma$ can be parameterized either as a normal graph of some function $w$ over $S$, using the vector field $N$, or as a graph of a different function $\bar{w}$ using the vector field $\bar{N}$. These functions are related by

$$
p+w(p) N(p)=\bar{p}+\bar{w}(\bar{p}) \bar{N}(\bar{p})=\phi(p, w(p))+\psi(p, w(p)) \bar{N}(\phi(p, w(p))) .
$$

Using the expansions above, we see that $\bar{w}(\bar{p})=w(p) /(N(p) \cdot \bar{N}(p))+O\left(\|w\|^{2}\right)$.

The mean curvature operators on these two functions, which we call $H_{w}$ and $\bar{H}_{\bar{w}}$, respectively, are related by

$$
\bar{H}_{\bar{w}}(\bar{p})=H_{w}(p) .
$$

Differentiating this with respect to $\bar{w}$ and setting $\bar{w}=0$, we get

$$
D_{\bar{w}} \bar{H}_{0}(u)=D_{w} H_{0}(\bar{N} \cdot N u)+\left(\nabla H_{0} \cdot \bar{N}_{t}\right) u,
$$

for any scalar function $u$. In the special case where the surface $\Sigma$ has constant mean curvature, this reduces to

$$
D_{\bar{w}} \bar{H}_{0}(u)=D_{w} H_{0}(\bar{N} \cdot N u)
$$

\section{Appendix II : Precise expansions for the mean curva- ture operator}

\subsection{Proof of the expansion (44)}

We use polar coordinates $(t, \theta)$ and write $u(t, \theta)=u\left(e^{t} \cos \theta, e^{t} \sin \theta\right)$. With this notation, (39) becomes $\nabla^{k} u(t, \theta)=\mathcal{O}\left(e^{-2 t}\right)$, where $\nabla$ is now the gradient with respect to $t$ and $\theta$. In the same way, the fact that the function $w$ as well as all its derivatives are assumed to be small means that $\nabla^{k} w(t, \theta)=\mathcal{O}\left(e^{-t}\right)$.

The mean curvature operator is

$$
H_{u+w}=e^{t} \nabla\left(\frac{e^{t} \nabla(u+w)}{\left(1+e^{2 t}|\nabla u+w|^{2}\right)^{1 / 2}}\right) .
$$

We start by noting that

$$
\frac{1}{\left(1+e^{2 t}|\nabla u+w|^{2}\right)^{1 / 2}}=\frac{1}{\left(1+e^{2 t}|\nabla u|^{2}\right)^{1 / 2}}-e^{2 t} \frac{\nabla u \cdot \nabla w}{\left(1+e^{2 t}|\nabla u|^{2}\right)^{3 / 2}}+Q_{1}\left(e^{t} \nabla w\right),
$$

where the function $Q_{1}(\cdot)$ is a function all of whose derivatives are bounded in $\mathcal{C}^{k}([-\log \rho,+\infty) \times$ $\left.S^{1}\right)$ and which satisfies $Q_{1}(0)=0$ and $\nabla Q_{1}(0)=0$.

Next, $H_{u+w}$ is given by

$$
e^{t} \nabla\left(\frac{e^{t}}{\left(1+e^{2 t}|\nabla u|^{2}\right)^{1 / 2}} \nabla(u+w)-e^{3 t} \nabla(u+w) \frac{\nabla u \cdot \nabla w}{\left(1+e^{2 t}|\nabla u|^{2}\right)^{3 / 2}}+e^{t} \nabla(u+w) Q_{1}\left(e^{t} \nabla w\right)\right) .
$$

From this it follows at once that

$$
H_{u+w}=H_{u}+\Lambda_{u} w-Q_{u}^{\prime}\left(e^{t} \nabla w, e^{t} \nabla^{2} w\right)-e^{t} Q_{u}^{\prime \prime}\left(e^{t} \nabla w, e^{t} \nabla^{2} w\right),
$$

where $Q_{u}^{\prime}(\cdot, \cdot)$ and $Q_{u}^{\prime \prime}(\cdot, \cdot)$ satisfy the required properties as stated in 444. 


\subsection{Proof of the expansion (11)}

We consider a surface parameterized by

$$
(s, \theta) \longrightarrow \mathbf{x}_{\varepsilon}^{c}(s, \theta)+\varepsilon \cosh s \tilde{w}(s, \theta) n(s, \theta),
$$

for some regular function $w(s, \theta)=\varepsilon \cosh s \tilde{w}(s, \theta)$, where $\mathbf{x}_{\varepsilon}^{c}$ is given by (6) and $n(s, \theta)$ is given by (8). A simple computation shows that the coefficients of the first fundamental form are then given by

$$
\begin{gathered}
E_{w}=\varepsilon^{2}\left(\cosh ^{2} s-2 \cosh s \tilde{w}+\cosh ^{2} s\left(\tilde{w}^{2}+\tilde{w}_{s}^{2}\right)+2 \sinh s \cosh s \tilde{w} \tilde{w}_{s}\right), \\
F_{w}=\varepsilon^{2}\left(\sinh s \cosh s \tilde{w}_{\theta} \tilde{w}+\cosh ^{2} s \tilde{w}_{s} \tilde{w}_{\theta}\right)
\end{gathered}
$$

and

$$
G_{w}=\varepsilon^{2}\left(\cosh ^{2} s+2 \cosh s \tilde{w}+\tilde{w}^{2}+\cosh ^{2} s \tilde{w}_{\theta}^{2}\right) .
$$

Notice that these can be written as

$$
\begin{gathered}
E_{w}=\varepsilon^{2}\left(\cosh ^{2} s-2 \cosh s \tilde{w}+\cosh ^{2} s P_{E}(\tilde{w}, \nabla \tilde{w})\right) \\
F_{w}=\varepsilon^{2} \cosh ^{2} s P_{F}(\tilde{w}, \nabla \tilde{w}) \\
G_{w}=\varepsilon^{2}\left(\cosh ^{2} s+2 \cosh s \tilde{w}+\cosh ^{2} s P_{G}(\tilde{w}, \nabla \tilde{w})\right)
\end{gathered}
$$

where $P_{E}, P_{F}$ and $P_{G}$ are polynomials, homogeneous of degree 2 , whose coefficients are bounded functions of $s$ and $\theta$. Moreover, using this we may write

$$
E_{w} G_{w}-F_{w}^{2}=\varepsilon^{4} \cosh ^{4} s\left(1+P_{E G-F^{2}}(\tilde{w}, \nabla \tilde{w})\right),
$$

Where $P_{E G-F^{2}}$ is a polynomial consisting of terms homogeneous of degree 2 or 4 , whose coefficients are bounded functions of $s$ and $\theta$.

In the same way, we compute the coefficients of the second fundamental form and find that these are given by

$$
\begin{gathered}
\sqrt{E_{w} G_{w}-F_{w}^{2}} e_{w}=-\varepsilon^{3}\left(\cosh ^{2} s+\cosh ^{3} s \tilde{w}_{s s}+\cosh ^{2} s \sinh s \tilde{w}_{s}+\cosh ^{2} s P_{e}\left(\tilde{w}, \nabla \tilde{w}, \nabla^{2} \tilde{w}\right)\right) \\
\sqrt{E_{w} G_{w}-F_{w}^{2}} f_{w}=-\varepsilon^{3}\left(\cosh ^{3} s \tilde{w}_{s \theta}++\cosh ^{2} s P_{f}\left(\tilde{w}, \nabla \tilde{w}, \nabla^{2} \tilde{w}\right)\right)
\end{gathered}
$$

and

$$
\begin{aligned}
\sqrt{E_{w} G_{w}-F_{w}^{2}} g_{w}=-\varepsilon^{3} \quad & -\cosh ^{2} s+\cosh ^{3} s \tilde{w}_{\theta \theta}+\left(\cosh ^{3} s-2 \cosh s\right) \tilde{w}+\cosh ^{2} s \sinh s \tilde{w}_{s} \\
& \left.+\cosh ^{2} P_{g}\left(\tilde{w}, \nabla \tilde{w}, \nabla^{2} \tilde{w}\right)\right),
\end{aligned}
$$

where, here also, $P_{e}, P_{f}, P_{g}$ are polynomials without any constant nor any linear terms and all of whose coefficients are bounded functions of $s$ and $\theta$.

The mean curvature operator may then be expressed in terms of these coefficients as

$$
H_{w}=\frac{e_{w} G_{w}-2 f_{w} F_{w}+g_{w} E_{w}}{E_{w} G_{w}-F_{w}^{2}}
$$

Using the previous expansions we obtain

$$
\begin{aligned}
H_{w}=-\frac{1}{\varepsilon \cosh s} \quad & \left(\tilde{w}_{s s}+\tilde{w}_{\theta \theta}+2 \tanh s \tilde{w}_{s}+\tilde{w}+\frac{2}{\cosh ^{2} s} \tilde{w}\right. \\
& \left.\frac{1}{\cosh s} P^{\prime}(\tilde{w})+P^{\prime \prime}(\tilde{w})\right)\left(1+\tilde{P}^{\prime}(\tilde{w})\right)
\end{aligned}
$$


where $P^{\prime}, \tilde{P}^{\prime}$ and $P^{\prime \prime}$ are functions of $\tilde{w}, \nabla \tilde{w}$ and $\nabla^{2} \tilde{w}$, all of whose partial derivatives are bounded functions in $\mathcal{C}^{k}\left(\left[-s_{\varepsilon}, s_{\varepsilon}\right] \times S^{1}\right)$, for all $k \geq 0$, uniformly in $\varepsilon$. Moreover, these functions satisfy

$$
\begin{gathered}
P^{\prime}(0,0,0)=\tilde{P}^{\prime}(0,0,0)=P^{\prime \prime}(0,0,0)=0 \\
\nabla P^{\prime}(0,0,0)=\nabla \tilde{P}^{\prime}(0,0,0)=\nabla P^{\prime \prime}(0,0,0)=0
\end{gathered}
$$

and $P^{\prime \prime}$ satisfies in addition

$$
\nabla^{2} P^{\prime \prime}(0,0,0)=0
$$

After having performed the change of function $\tilde{w}=w /(\varepsilon \cosh s)$, we conclude that

$$
\begin{aligned}
H_{w}=-\frac{1}{\varepsilon^{2} \cosh ^{2} s} \mathcal{L} w & +\frac{1}{\varepsilon \cosh ^{2} s} Q_{\varepsilon}^{\prime}\left(\frac{w}{\varepsilon \cosh s}, \frac{\nabla w}{\varepsilon \cosh s}, \frac{\nabla^{2} w}{\varepsilon \cosh s}\right) \\
& +\frac{1}{\varepsilon \cosh s} Q_{\varepsilon}^{\prime \prime}\left(\frac{w}{\varepsilon \cosh s}, \frac{\nabla w}{\varepsilon \cosh s}, \frac{\nabla^{2} w}{\varepsilon \cosh s}\right),
\end{aligned}
$$

where $Q_{\varepsilon}^{\prime}$ and $Q_{\varepsilon}^{\prime \prime}$ are functions all of whose partial derivatives are bounded in $\mathcal{C}^{k}\left(\left[-s_{\varepsilon}, s_{\varepsilon}\right] \times S^{1}\right)$, for all $k \geq 0$, uniformly in $\varepsilon$. Moreover, these functions satisfy

$$
Q_{\varepsilon}^{\prime}(0,0,0)=Q_{\varepsilon}^{\prime \prime}(0,0,0)=0 \quad \text { and } \quad \nabla Q_{\varepsilon}^{\prime}(0,0,0)=\nabla Q_{\varepsilon}^{\prime \prime}(0,0,0)=0 .
$$

and $Q_{\varepsilon}^{\prime \prime}$ satisfies in addition

$$
\nabla^{2} Q_{\varepsilon}^{\prime \prime}(0,0,0)=0
$$

\section{Appendix III : Mapping properties of the Laplace op- erator in a cylinder}

We collect here various results whose proofs are slight modifications of the proof of Proposition 4 .

Lemma 4 Assume that $\delta \in(1,2)$ and that $0<s_{0}<s_{1}$. Then, there exists some operator

$$
G_{s_{0}, s_{1}}: \mathcal{C}_{\delta+2}^{0, \alpha}\left(\left[s_{0}, s_{1}\right] \times S^{1}\right) \longrightarrow \mathcal{C}_{\delta}^{2, \alpha}\left(\left[s_{0}, s_{1}\right] \times S^{1}\right),
$$

such that, for all $f \in \mathcal{C}_{\delta+2}^{0, \alpha}\left(\left[s_{0}, s_{1}\right] \times S^{1}\right)$, the function $w=G_{s_{0}, s_{1}}(f)$ is a solution of the problem

$$
\left\{\begin{array}{rlll}
e^{2 s} \Delta w & =f & \text { in } & \left(s_{0}, s_{1}\right) \times S^{1} \\
\pi^{\prime \prime} w=0 & \text { on } & \left\{s_{1}\right\} \times S^{1} \\
w=0 & \text { on } & \left\{s_{0}\right\} \times S^{1} .
\end{array}\right.
$$

In addition, we have $\left\|G_{s_{0}, s_{1}}(f)\right\|_{2, \alpha, \delta} \leq c\|f\|_{0, \alpha, \delta+2}$, for some constant $c>0$ independent of $s_{0}, s_{1}$.

Proof : Using separation of variables as in the proof of Proposition 4, we now write

$$
\tilde{w}=\sum_{n \in \mathbb{Z}} w_{n}(s) e^{i n \theta} \quad \text { and } \quad f=e^{2 s} \sum_{n \in \mathbb{Z}} f_{n}(s) e^{i n \theta} .
$$

By linearity, we may assume that $|f|(s) \leq e^{(\delta+2) s}$ and therefore we find $\left|f_{n}\right|(t) \leq e^{\delta s}$. This time, for all $|n| \geq 2$, we see that $w_{n}$ has to solve

$$
\ddot{w}_{n}-n^{2} w_{n}=f_{n} \quad \text { in } \quad\left(s_{0}, s_{1}\right),
$$


and $w_{n}\left(s_{0}\right)=w_{n}\left(s_{1}\right)=0$. It is easy to see that the function $\frac{2}{n^{2}-\delta^{2}} e^{\delta s}$ is a supersolution for our problem therefore this yields, for all $|n| \geq 2$

$$
\left|w_{n}\right|(s) \leq \frac{2}{n^{2}-\delta^{2}} e^{\delta s}
$$

For $n=0$ and $n= \pm 1$, we use the explicit formula

$$
w_{0}(s)=\int_{s_{0}}^{s} \int_{s_{0}}^{t} f_{0}(u) d u d t \quad \text { and } \quad w_{ \pm 1}(s)=e^{-s} \int_{s_{0}}^{s} e^{2 t} \int_{s_{0}}^{t} e^{-u} f_{ \pm 1}(u) d u d t .
$$

Summation over $n$ and Schauder's estimates lead to the desired result.

Our next Lemma is a variant of the previous result.

Lemma 5 Assume that $\delta \in(0,1)$ and that $0<s_{0}<s_{1}$. Then, there exists some operator

$$
G_{s_{0}, s_{1}}^{\prime}: \mathcal{C}_{\delta+2}^{0, \alpha}\left(\left[s_{0}, s_{1}\right] \times S^{1}\right) \longrightarrow \mathcal{C}_{\delta}^{2, \alpha}\left(\left[s_{0}, s_{1}\right] \times S^{1}\right),
$$

such that, for all $f \in \mathcal{C}_{\delta+2}^{0, \alpha}\left(\left[s_{0}, s_{1}\right] \times S^{1}\right)$, the function $w=G_{s_{0}, s_{1}}^{\prime}(f)$ is a solution of the problem

$$
\left\{\begin{array}{rlll}
e^{2 s} \Delta w=f & \text { in } & \left(s_{0}, s_{1}\right) \times S^{1} \\
\pi^{\prime} w=0 & \text { on } & \left\{s_{1}\right\} \times S^{1} \\
w=0 & \text { on } & \left\{s_{0}\right\} \times S^{1} .
\end{array}\right.
$$

In addition, we have $\left\|G_{s_{0}, s_{1}}^{\prime}(f)\right\|_{2, \alpha, \delta} \leq c\|f\|_{0, \alpha, \delta+2}$, for some constant $c>0$ independent of $s_{0}, s_{1}$.

Proof : The only difference with the proof of the previous result is that, this time $\frac{2}{n^{2}-\delta^{2}} e^{\delta s}$ is a supersolution for our problem for all $|n| \geq 1$.

We will also need

Lemma 6 Assume that $\delta^{\prime} \in(-1,0)$ and that $s_{0}>0$. Then, there exists an operator

$$
\hat{G}_{s_{0}}: \mathcal{C}_{\delta^{\prime}+2}^{0, \alpha}\left(\left[s_{0},+\infty\right) \times S^{1}\right) \longrightarrow \mathcal{C}_{\delta^{\prime}}^{2, \alpha}\left(\left[s_{0},+\infty\right) \times S^{1}\right) \oplus \mathbb{R},
$$

such that, for all $f \in \mathcal{C}_{\delta^{\prime}+2}^{0, \alpha}\left(\left[s_{0},+\infty\right) \times S^{1}\right)$, the function $w=\hat{G}_{s_{0}}(f)$ is the unique solution of the problem

$$
\left\{\begin{array}{rll}
e^{2 s} \Delta w=f & \text { in } \quad\left(s_{0},+\infty\right) \times S^{1} \\
w=0 & \text { on } \quad\left\{s_{0}\right\} \times S^{1},
\end{array}\right.
$$

which belongs to the space $\mathcal{C}_{\delta^{\prime}}^{2, \alpha}\left(\left[s_{0},+\infty\right) \times S^{1}\right) \oplus \mathbb{R}$. In addition, if we decompose $w(s, \theta)=$ $v(s, \theta)+c_{0} \in \mathcal{C}_{\delta^{\prime}}^{2, \alpha}\left(\left[s_{0},+\infty\right) \times S^{1}\right) \oplus \mathbb{R}$, we have $e^{-\delta^{\prime} s_{0}}\left|c_{0}\right|+\|v\|_{2, \alpha, \delta^{\prime}} \leq c\|\tilde{f}\|_{0, \alpha, \delta^{\prime}+2}$, for some constant $c>0$ independent of $s_{0}$.

Proof : Again, using separation of variables as in the proof of Lemma 1 , we write

$$
w=\sum_{n \in \mathbb{Z}} w_{n}(s) e^{i n \theta} \quad \text { and } \quad f=e^{2 s} \sum_{n \in \mathbb{Z}} f_{n}(s) e^{i n \theta} .
$$

By linearity, we may assume that $|f|(s) \leq e^{\left(\delta^{\prime}+2\right) s}$ and therefore we find $\left|f_{n}\right|(t) \leq e^{\delta^{\prime} s}$. Here, for all $|n| \geq 1$, we see that $w_{n}$ has to solve

$$
\ddot{w}_{n}-n^{2} w_{n}=f_{n} \quad \text { in } \quad\left(s_{0},+\infty\right),
$$


and $w_{n}\left(s_{0}\right)=0$. It is easy to see that the function $\frac{2}{n^{2}-\left(\delta^{\prime}\right)^{2}} e^{\delta^{\prime} s}$ is a supersolution for our problem therefore this yields, for all $|n| \geq 1$

$$
\left|w_{n}\right|(s) \leq \frac{2}{n^{2}-\left(\delta^{\prime}\right)^{2}} e^{\delta^{\prime} s}
$$

For $n=0$, the variation of the constant formula provides us with the explicit formula

$$
w_{0}(s)=c_{0}+\int_{s}^{+\infty} \int_{t}^{+\infty} f_{0}(u) d u d t, \quad \text { with } \quad c_{0}=-\int_{s_{0}}^{+\infty} \int_{t}^{+\infty} f_{0}(u) d u d t .
$$

And the desired estimates follow at once by summation over $n$ and direct estimate for $c_{0}$.

Using similar arguments, we can also prove

Lemma 7 There exists an operator

$$
\mathcal{P}: \pi^{\prime \prime}\left(\mathcal{C}^{2, \alpha}\left(S^{1}\right)\right) \longrightarrow \mathcal{C}_{-2}^{2, \alpha}\left((-\infty, 0] \times S^{1}\right),
$$

such that, for all $\phi^{\prime \prime} \in \pi^{\prime \prime}\left(\mathcal{C}^{2, \alpha}\left(S^{1}\right)\right)$, the function $w=\mathcal{P}\left(\phi^{\prime \prime}\right)$ is the unique solution of

$$
\left\{\begin{array}{rlrl}
\Delta w & =0 & \text { in } & (-\infty, 0) \times S^{1} \\
w=\phi^{\prime \prime} & \text { on } & \{0\} \times S^{1},
\end{array}\right.
$$

which belongs to the space $\mathcal{C}_{-2}^{2, \alpha}\left((-\infty, 0] \times S^{1}\right)$. In addition, we have $\left\|\mathcal{P}\left(\phi^{\prime \prime}\right)\right\|_{2, \alpha,-2} \leq c\left\|\phi^{\prime \prime}\right\|_{2, \alpha}$, for some constant $c>0$.

Proof : As in the proof of the previous Lemma, we decompose $\phi^{\prime \prime}$ into Fourier series $\phi^{\prime \prime}=$ $\sum_{|n| \geq 2} \phi_{n} e^{i n \theta}$. The solution $w$ is then explicitly given by $w=\sum_{|n| \geq 2} \phi_{n} e^{|n| s} e^{i n \theta}$, from which it immediately follows that

$$
|w|(s) \leq 2 e^{2 s}\left(1+\sum_{n \geq 3} e^{(n-2) s}\right)\|\phi\|_{2, \alpha} .
$$

Therefore, we already obtain $\sup _{s \leq-1} e^{-2 s}|w|(s) \leq c\left\|\phi^{\prime \prime}\right\|_{2, \alpha}$. It also follows from the explicit formula for $w$ that $\|w(-1, \cdot)\|_{\mathcal{C}^{2, \alpha}} \leq c\left\|\phi^{\prime \prime}\right\|_{2, \alpha}$. Using this last estimate as well as the fact that $\Delta w=0$ in $(-1,0) \times S^{1}$, we find from Schauder's estimates that $\sup _{s \in(-1,0)} e^{-2 s}|w|(s) \leq c\left\|\phi^{\prime \prime}\right\|_{2, \alpha}$. The other estimates, for the derivatives of $w$, follow again from Schauder's estimates.

\section{References}

[1] C. Delaunay, Sur la surface de revolution dont la courbure moyenne est constante, J. de Mathématiques, 6 (1841) 309-320.

[2] D. Gilbarg and N. Trudinger, Elliptic partial differential equations of second order, Second edition. Grundlehren der Math. Wiss. 224 Springer-Verlag, Berlin-New York, 1983.

[3] K. Grosse-Brauckmann, R. Kusner and J. Sullivan, Constant mean curvature surfaces with three ends, MSRI preprint \# DG/9903101.

[4] N. Kapouleas, Complete constant mean curvature surfaces in Euclidean three space, Ann. of Math. (2) 131 (1990), 239-330.

[5] N. Kapouleas Compact constant mean curvature surfaces in Euclidean three-space, J. Diff. Geom. 33 (1991), 683-715. 
[6] N. Kapouleas Constant mean curvature surfaces constructed by fusing Wente tori, Invent. Math. 119 (1995), 443-518.

[7] N. Kapouleas Complete embedded minimal surfaces of finite total curvature, J. Diff. Geom. 47 (1997) 95-169.

[8] R. Kusner, R. Mazzeo and D. Pollack, The moduli space of complete embedded constant mean curvature surfaces, Geom. Funct. Anal. 6 (1996) 120-137.

[9] R. Mazzeo and F. Pacard Constant mean curvature surfaces with Delaunay ends, Preprint, 1998.

[10] R. Mazzeo, F. Pacard and D. Pollack Complete surfaces of constant mean curvature: connected sums and moduli spaces, In preparation.

[11] R. Mazzeo and D. Pollack, Gluing and moduli for some noncompact geometric problems, in Geometric Theory of Singular Phenomena in Partial Differential Equations, Symposia Mathematica Vol XXXVIII, Cambridge Univ. Press (1998), 17 - 51.

[12] R. Mazzeo, D. Pollack and K. Uhlenbeck Connected sum constructions for constant scalar curvature metrics, Topol. Methods Nonlinear Anal. 6 (1995), no. 2, 207-233.

[13] S.D. Yang, A connected sum construction for complete minimal surfaces with finite total curvature, Preprint. 